\title{
The Impact of Socio-cultural Issues on Mature Women and Alcohol Use in Glasgow, Scotland: An Anthropological Exploration.
}

by

Angeline M. Jones

A thesis submitted to the Faculty of Graduate and Postdoctoral Affairs in partial fulfillment of the requirements for the degree of

Master of Arts

in

Anthropology

Carleton University

Ottawa, Ontario

(C) 2013, Angeline M. Jones 


\section{Abstract}

This thesis explores the connection between women's health and alcohol consumption in Glasgow, Scotland. The purpose of the project is to investigate how social, economic and other factors affect women's alcohol consumption, and whether or not female alcohol consumption has a direct effect on women's ill-health in Glasgow, Scotland. The data gleaned from participant observation and semi-structured interviewing of thirteen Glaswegian women provide evidence that female alcohol consumption is inseparable from issues of gender, identity, class, and power. The data will be examined using three unique levels of analysis: the individual, the societal, and the institutional. Such an examination helps to capture the lived reality of Glaswegian women. Furthermore, it situates women's alcohol use in the contemporary context of Scotland's political economy. Finally, the thesis concludes with a discussion of women's alcohol use in Glasgow and its connection to the 'Glasgow Effect'. 


\section{Acknowledgements}

This research project would not have been possible without the time and energy of thirteen vibrant, dynamic and wonderfully open women from Glasgow, Scotland. I extend my heartfelt gratitude to them all for taking the time to help out a first time fieldworker and for sharing their experiences and thoughts with me.

I would also like to extend a warm thank you to Dr. Jen Pylypa for her help with revisions and for participating in my committee.

Dr. Louise de la Gorgendiere provided me with the direction and support I required to embark on this degree, and this project, and I wish to thank her for her guidance and expertise in leading me down the (sometimes thorny, but always rewarding) path to the completion of a MA thesis in Anthropology. Thank you for believing in me.

Funding for this project was provided in part by Carleton University, the European Union, and the Centre for European Studies.

Finally, I would like to thank my husband, Mark, who lent an ear when needed, traveled with me to Glasgow, and encouraged me to greater work with his patience and positivity. You remain my favourite partner in crime. 


\section{Table of Contents}

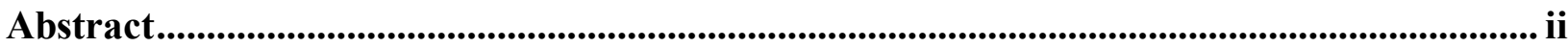

Acknowledgements .................................................................................................................................. iii

Table of Contents ............................................................................................................................. iv

List of Tables ............................................................................................................................................ v

List of Illustrations....................................................................................................................... vi

List of Appendices................................................................................................................................ vii

Chapter One: Introduction to the Research Project ........................................................................... 1

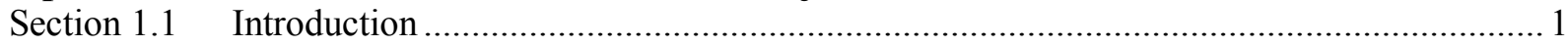

Section 1.2 Significance of the research: importance to the field and society ................................... 3

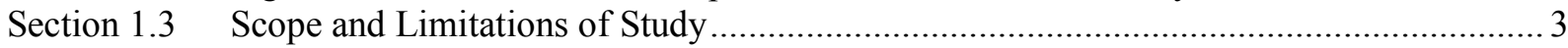

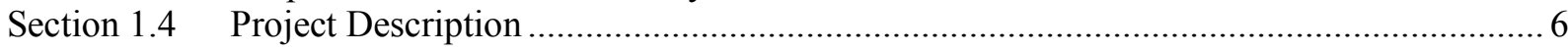

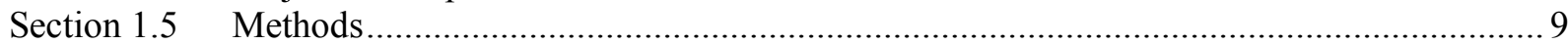

Section 1.6 A brief history of Glasgow ………………........................................................ 11

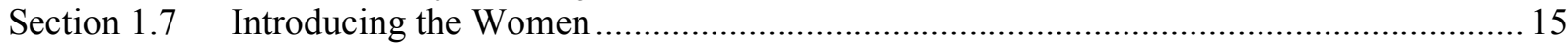

Section 1.8 Summary \& Chapter Outline ................................................................................ 23

Chapter Two: Anthropology and the Study of Female Alcohol Use ........................................... 25

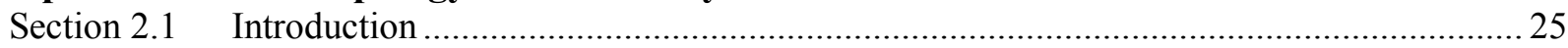

Section 2.2 Anthropology and A Woman's Place in Alcohol Studies .............................................25

Section $2.3 \quad$ Gender, Class and Identity..................................................................................... 30

Section 2.4 A Critical Medical Anthropological Perspective ............................................................. 39

Section 2.5 Power and the Critical Medical Anthropological Gaze..................................................... 45

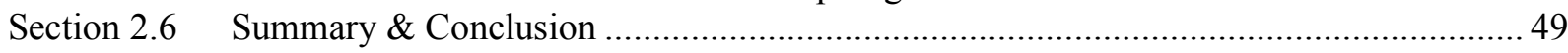

Chapter Three: Women's Drinking and Sociocultural Factors .................................................5 50

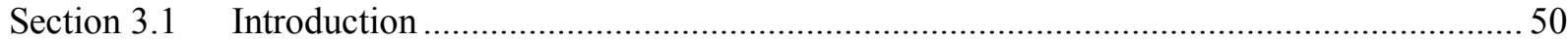

Section 3.2 Glasgow as a hard drinking place ………………...................................................... 51

Section 3.3 Then and Now - the changing landscape of women's drinking ....................................5 55

Section 3.4 Aging and its implication for women's drinking behaviours ..........................................6 60

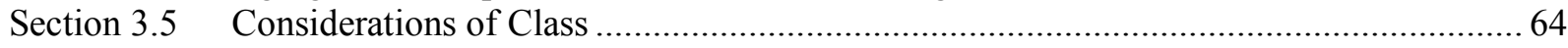

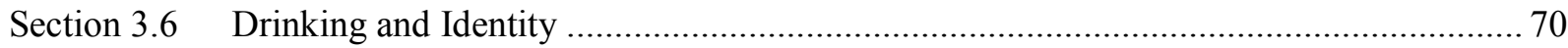

Section 3.7 Summary \& Conclusion ........................................................................................ 74

Chapter Four: Health, Alcohol and Political Economy in Scotland ..........................................76

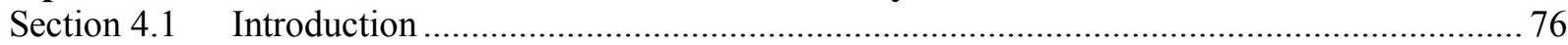

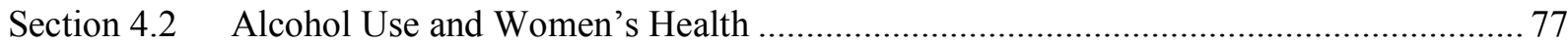

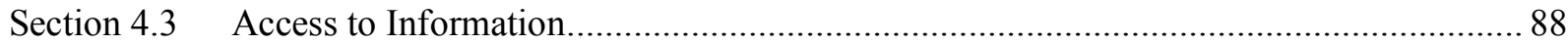

Section 4.4 Accessibility of Alcohol and Understanding How Much is too Much............................ 92

Section 4.5 Political Economy \& Scotland - What's Alcohol Got to do with it? ............................ 97

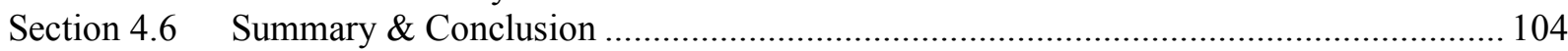

Chapter Five: Summary and Conclusions........................................................................................ 105

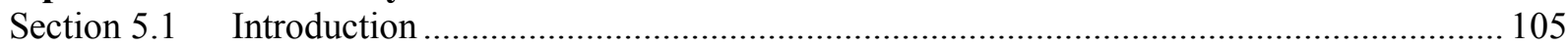

Section 5.2 Three Levels of Analysis and Women's Alcohol Consumption ................................... 106

Section 5.3 Women's Alcohol Use and The Glasgow Effect ...................................................... 115

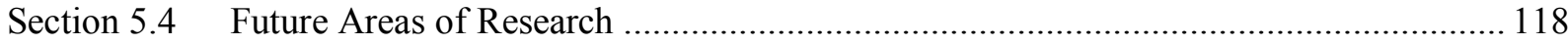

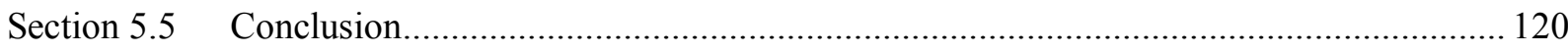




\section{List of Tables}

Table 1 Demographic information of Participants..................................................... 7

Table 2 NHS Scotland table of 'safe' drinking levels................................................. 94 


\section{List of Illustrations}

Figure 1 An alcohol awareness poster on a bus shelter in Glasgow targeting female

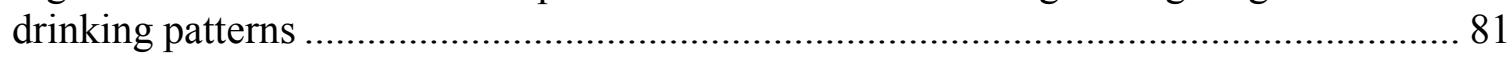

Figure 2 Commentary from a citizen regarding a new bar in the Scottish Parliament..... 98

Figure 3 A sign in an upmarket grocery store advertising a dinner special that includes a

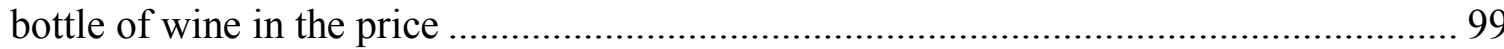

Figure 4 An article regarding high drinking levels in Scotland.................................... 101 


\section{List of Appendices}

Appendix A Interview Questions................................... 133 


\section{Chapter One: Introduction to the Research Project}

\section{Section 1.1 Introduction}

In recent decades Scotland has presented as the region with the highest mortality rates and the lowest life expectancy in all of Western Europe (Walsh et al. 2010a: 10). When Hanlon et al. (2005) first explored the higher mortality in Scotland compared to England and Wales, this phenomenon was labelled as the 'Scottish Effect'. Further research found that within Scotland itself the worst health indicators overall were located in the west central region of the country and, in particular, in the largest urban centre located in that region, Glasgow (Walsh et al. 2010b: 487). Because of this information the phenomenon is now referred to as the 'Glasgow Effect' (Ibid).

The idea that health is both influenced and determined by certain social factors, such as poverty and (un)employment, is now accepted in most public health realms. The World Health Organization (WHO 2012) states that these conditions are created through policy decisions at the local, regional, national and international levels. What is perhaps most interesting about the 'Glasgow Effect' is that the poor health indicators found in Glasgow do not appear to be caused by deprivation alone (Walsh et al. 2010b). Furthermore, this excess mortality is confined not only to poorer regions of the city, but is seen across the population; although premature mortality rates are higher in deprived areas with over half related to alcohol and drugs (Ibid: 493). A study of gender differences in the geography of alcohol related mortality found that "many areas with the highest alcohol-related mortality rates were located within Greater Glasgow" (Emslie \& Mitchell 2009: n.p.). The 'Glasgow Effect' is of further concern and requires important consideration as it also appears to be a recent phenomenon, with the mortality gap widening since the 1970s (Walsh et al. 2010b: 488). There have been a variety of factors considered to 
explain this 'effect', including internal migration patterns, problems with the methodologies used to capture all aspects of socio-economic deprivation, genetic factors, lack of vitamin D, possible family breakdown and some aspects generally attributed to Scottish culture (Ibid; Bloor, et al. 2008; Popham et al. 2011). It is this last factor which will help frame the subject matter of my research project: Scottish culture and alcohol consumption.

Seaman and Edgar (2012) note in their study of youth drinking behaviours and gender, that Scotland has a recent history of attempting to address the nation's relationship with alcohol. This is due, in part, to the fact that alcohol related deaths have doubled in the country in the past 15 years, thereby making alcohol issues a priority for the Scottish Government (Ibid: 7). There has also been a rise in the rate of young men and women's alcohol consumption in the country over the past few decades (Ibid: 8). The rise in women's drinking is not a phenomenon that is specific to Scotland, however, but one that is being seen across many Western countries such as New Zealand, England, the United States and multiple countries in Europe (Gordon et al. 2012; Lyons \& Willott 2008; Rudolfsdottir \& Morgan 2009; Thom 1997; Zilberman et al. 2008). Changing patterns in female drinking is an area of study that may provide insight into women's well-being and health within the Scottish context, and may reveal social and cultural factors that contribute to the poor health outcomes found in the West of Scotland. This chapter provides information regarding the significance of the research, its scope and limitations, a detailed project description, a discussion of the methodologies used, a brief history of Glasgow, and an introduction to the participants who volunteered to be interviewed for this project. The chapter concludes with a brief description of the subsequent chapters. 


\section{Section 1.2 Significance of the research: importance to the field and society}

This research project examined sociocultural factors related to female drinking in Glasgow and explored how women in Glasgow frame their experiences and relationship with alcohol use. The purpose of the project was to investigate how social, economic and other factors impinge on women's alcohol consumption, and whether or not female drinking has a direct effect on Scotland's health. It is only through reflection on a multitude of factors that anthropologists can ensure they have a clear view of all influences and circumstances in a particular cultural setting. More research is needed to determine if, in fact, women's drinking is one aspect of causality to be considered when discussing the 'Glasgow Effect', and this project takes the first step in this process. Findings from this study contribute information to fill existing gaps in the anthropological literature on alcohol studies and women and, in particular, alcohol consumption and mature women ${ }^{1}$ in Glasgow.

\section{Section 1.3 Scope and Limitations of Study}

The purpose of my research project was to investigate mature women's experiences with, and attitudes towards, female drinking ${ }^{2}$ in Glasgow, Scotland. I explored what women from this community know about the effects of alcohol use, and how alcohol use may be implicated in their daily lives. The analysis of the data of this study resulted in the emergence of themes related to gender, identity and class. The findings from this study support the argument that alcohol consumption carries the weight of hegemonic discourses of masculinity and femininity, and can be used to support, or resist, these discourses. I also argue that identity and class are inextricably linked to alcohol consumption in Glasgow, and the relationships between these sociocultural factors dictates behaviour and societal perceptions. This thesis also investigates

\footnotetext{
${ }^{1}$ Within the context of this thesis, the term 'mature women' refers to any female over the age of 25 years.

${ }^{2}$ Within the context of this thesis, the term 'drinking' should be understood to mean 'drinking alcohol' unless otherwise indicated.
} 
links between women's health and alcohol consumption, the political climate in which these connections occur and their relevance to the 'Glasgow Effect'. The link between women's alcohol use and its connection to female health is largely ignored by the participants of this study. The reasons for this are related to historical, gendered expectations of alcohol use; changes that occur over the female lifecourse; gendered power relations; and, the fact that female public drinking is a relatively new phenomenon and, thus, there has not yet been time to build easily recognizable connections between women's alcohol use and female health. I also explore alcohol use from a political economy perspective to illustrate the intersection of a variety of competing factions that link alcohol use to other facets of society, such as public health and industry demands in Scotland and the European Union.

Further analysis of the key themes mentioned above reveals that they must be considered from within a context of existing power relations that are manifest in the Glaswegian context. These relations are found at the personal, societal and larger institutional levels, each of which has a direct impact on the participants' lives. These themes and considerations will be explored in the following chapters.

The data arising from this study must be considered within the limitations of the research experience. The first limitation was the limited amount of time I was able to remain in Glasgow. Three months, while enough time to meet people and recruit volunteers to the study, does not allow enough time to become involved in every aspect of a participant's life. As such, participants of this study may have been met only once, or within a specific context where I was already designated a role (i.e. volunteer at a non-profit, community based organization). This meant that while I could gather information through the interview process, I was unable to compare this data to observations of the same people in a variety of contexts to assess their 
behaviour in relation to what had been said during interviews. I was also unable to view the participants in a variety of contexts to see how their behaviour adapted, for example, when at home, among close friends, or when out socializing. Further access to occasions such as these would have provided me with additional insight and an ability to compare data from a variety of contexts. The second limitation was the small number of people I was able to interview. While the background of the thirteen women I interviewed provides a range of socioeconomic levels, marital status, and types of education completed, I was unable to recruit anyone who could be said to be living within the lowest socioeconomic class in Glasgow, that is, those from the poorest regions and backgrounds. As a result, this important viewpoint remains unaccounted for within this study. The third limitation was related to the fact that some of the participants were employed in non-profit organizations that were familiar with alcohol issues or conducting research in public health. This means that these participants may have had a heightened awareness of issues related to alcohol use greater than that found in the general population. The fourth limitation was the relationships between those who participated in group interviews. Those who agreed to be interviewed in this way were either work colleagues or neighbourhood friends who, as I deduced during the interview process, did not have intimate friendships. This means that the participants may have been more guarded in their responses due to fear of revealing details that were too personal given the level of intimacy between those being interviewed. This also meant that participants might have left issues unsaid, or gone along with comments stated by others in the group as a means to protect their own interests. The fifth limitation I encountered was my previous experience of living in Scotland. I lived in Edinburgh for several years, and in Oban on the west coast of Scotland, though not in Glasgow itself. This previous exposure to Scotland meant that I took for granted certain aspects of culture and the environment in which I found myself as it was already familiar to me. Because of this, I 
sometimes had to double back through my notes to ensure I had captured observations and experiences that would be useful or informative, as on first exposure I might have overlooked certain incidents or key points for analysis. Lastly, the final limitation of note is my lack of previous experience as a social researcher in the field. This led me to underestimate certain variables such as: how long it would take me to recruit volunteers; how to actually write up useful field notes; and, how to overcome initial shyness when seeking recruits for the study. As a result, I chose to view the fieldwork experience as a work in progress, trying things out to see what worked, and revising my thoughts and actions as needed to procure the information and create the experiences I needed to ensure I had enough data. Despite these limitations, I am confident that the data I collected are useful and noteworthy, as I will show in the following chapters. To provide further context I now turn to a description of the research project itself.

\section{Section 1.4 Project Description}

The total number of women interviewed for this study was thirteen. Of these women, four identified as single and nine identified as married. There were two group interviews comprised of four women each, while five participants were interviewed individually. Of these thirteen participants, ten had children and three did not. All participants had lived in Glasgow for a minimum of five years. Ages ranged from 25 to 65, and education levels spanned from completion of school (equivalent to graduating high school in Canada) to $\mathrm{PhD}$ degrees. These data serve to demonstrate that the participants came from a variety of socio-economic backgrounds, and were at varying stages in the life course, thereby providing a range of experiences on which to draw and compare responses. All participants were required to sign consent forms and their anonymity was assured. Ethical approval for the project was provided by 
the Research Ethics Board, at Carleton University, in Ottawa, Ontario, Canada. The table below summarizes the demographic data collected from the participants.

\begin{tabular}{|l|l|l|l|l|}
\hline $\begin{array}{l}\text { Marital Status (self- } \\
\text { identified) }\end{array}$ & $\begin{array}{l}\text { Type of } \\
\text { Interview }\end{array}$ & $\begin{array}{l}\text { Age } \\
\text { Category }\end{array}$ & Level of Education & Pseudonym \\
\hline Single & Group & $25-34$ & Master's Degree & Deirdre \\
\hline Married & Group & $25-34$ & Master's Degree & Roxanne \\
\hline Married & Group & $35-44$ & Bachelor's Degree & Joanne \\
\hline Married & Group & $35-44$ & HND $^{3}$ & Lila \\
\hline Married & Group & $35-44$ & Degree (unspecified) & Chloe \\
\hline Married & Group & $35-44$ & PhD & Megan \\
\hline Married & Group & $35-44$ & Highers & Mary \\
\hline Married & Group & $35-44$ & PhD & Iris \\
\hline Married & Individual & $35-44$ & Degree (unspecified) & Lena \\
\hline Single & Individual & $45-54$ & Highers & Bea \\
\hline Married & Individual & $55-65$ & Registered General Nurse & Corinne \\
\hline Single & Individual & $25-34$ & HND & Beth \\
\hline Single & Individual & $35-44$ & Master's Degree & Cilia \\
\hline
\end{tabular}

Table 1 Demographic information of Participants

To recruit participants I visited pubs, cafes, shops and local libraries to post recruitment posters and explain to employees the purpose of my research project. I was also able to draw on the connections I made with both the organization where I volunteered while living in Glasgow, and the non-profit organization that I had contacted prior to arriving in Glasgow. Employees and volunteers at these organizations were extremely helpful in offering to circulate my recruitment flyer to others, and in agreeing to participate in the interview process themselves. I received written approval from the charity where I volunteered that explained that they knew of my

\footnotetext{
${ }^{3}$ Higher National Certificates and Higher National Diplomas (HND) are vocational higher education qualifications. In Scotland, an HNC is awarded at the end of the first year of a two-year HND course; it is also a qualification in its own right. Unlike in the rest of the UK where they are often studied part time, HNCs in Scotland are full-time, one-year programmes. In many cases, an HNC can allow you to progress to the second year of an undergraduate degree, while an HND may let you move into the third year of a degree. http://www.educationuk.org/Article/GB1262438024220

${ }^{4}$ Highers are normally taken at the end of the fifth year of secondary education, at the age of 17 . Some pupils will have passed four, five or even six Highers by the end of the fifth year. Highers are the qualifications most often required for entry to higher education courses in Scotland. http://www.educationuk.org/Article/GB1262438024220
} 
research project and supported it wholeheartedly. As an incentive, I offered to provide refreshments at the group interviews (i.e. cakes, and non-alcoholic drinks, sandwiches), and a $£ 10$ gift card to a local drugstore for individual interviews. Due to ethical considerations, when choosing a retailer from whom to purchase the gift cards, I was careful not to choose a place that sold alcohol as I felt this presented a conflict of interest.

All interviews were audio recorded and, with the participant's consent, I also took written notes as a safeguard against technical glitches with the digital recording device. Interviews were held in a variety of locations which I describe later in this chapter when I present more information on the women who were interviewed. The interviews were transcribed using Express Scribe version 5.5, NCH Software, and, in several instances, by employing the Voice Transcription Technique with Dragon Naturally Speaking Software version 11.5 (Matheson 2007). The data was analyzed using a thematic approach. This required multiple readings of the transcriptions. I used open coding in the first reading of all transcriptions, adding brief notes to the pages as I went through (Ryan \& Bernard 2003: 88). These notes were then reviewed to ascertain dominant themes that appeared across the interviews. These themes were colour-coded using coloured cue cards, and arranged on foam boards to separate the content. Cue cards also contained information that identified the interview that was being referenced and the line or page number on the transcript where the information could be easily found later during the writing process. Further reading of the transcripts was required to identify sub-themes that arose from the dominant themes. Identified sub-themes were also colour-coded and placed on the foam boards, relative to the dominant theme to which they were related. This allowed me to identify a variety of themes, and to have a visual representation of their relationships to one another that proved very helpful in the analytical process. The next section provides details regarding the research methods used to capture the data. 


\section{Section 1.5 Methods}

For this project I chose a variety of research methods. The first was participant observation (Fetterman 2010). I lived in a neighbourhood in Glasgow for three months, and to make initial contact with community members I volunteered at a local charity shop. Prior to arriving in Glasgow, I had also made contact with another not-for-profit organization that had published a variety of reports on the 'Glasgow Effect'. The relationships I cultivated with both these organizations were invaluable as they helped me to make initial contact with community members, and aided me greatly when I began recruiting volunteers for the study. When not volunteering, I spent my time in the community, observing behaviours of those around me in public places, and using field notes to record what I observed. Forsey (2010: 560) draws on the importance of participant listening in ethnography and argues for 'engaged listening' to be placed on "a similar footing to participant observation". Arguing that participant observation is as much about what we hear as about what we observe, he believes in a 'democracy of the senses' so that all information can be included and processed (Ibid). This argument also applies to the use of interviewing as a methodological tool in the field, and elucidates that it is as effective and methodologically sound as other types of ethnography (Ibid). I used semistructured interviewing and held both individual and group interviews (Bernard 2011). Forsey (2010: 567) states that conducting an interview with an "ethnographic imaginary is aimed at revealing the cultural context of individual lives". This 'ethnographic imaginary' requires that interview questioning is flexible enough that it can be used to elicit information beyond the direct interest of the research question (Ibid). That is, employing not only those questions that relate simply to one direct request for information, but also including questions that frame the thoughts and processes that occurred around a particular action (Ibid). Such a form of 
questioning elicits information from within a cultural context that adds robustness to, and a deeper understanding of, the data.

I also considered the work of Scholte (1969), which calls on anthropology's emancipatory and normative interests in order to create a more self-reflective practice. The employment of comparative understanding of others leads to self-awareness, self-reflection and self-emancipation, thusly allowing anthropologists to understand others more fully and enter into solidarity with them (Ibid). Self-reflection is crucial to this process and holds the promise of unveiling further linkages and deeper understandings of the cultural implications of these connections that will result in a more focused and informative anthropological view. To this end, I also kept a personal journal to document my own impressions of my time in the field, including personal notes on the challenges I encountered, initial impressions of Glasgow, and the gamut of emotions I encountered while learning how to 'be an anthropologist' and 'do fieldwork'. These personal reflections have been extremely helpful to me in the writing of this thesis and remind me of the personal transformation that takes place when engaging in fieldwork for the first time.

For my research project I chose these specific methods for several reasons. Firstly, participant observation is at the heart of anthropological fieldwork and allowed me to capture observations of both verbal and non-verbal behaviour. Secondly, semi-structured interviewing allowed me to be well-prepared in one-to-one interviews, as I employed an interview script which guided the process, but still left room for participants to expand on topics related to the general subject matter that were of particular importance to them (Bernard 2011: 157-158). Lastly, the reflections captured in my personal journal allowed me to review the unfolding of the fieldwork process from start to finish, including how it affected my thought processes and the steep learning curve which I experienced. In short, my writings serve to remind me of where I 
started and how I ended up here. The next section reproduces some of these impressions, coupled with factual accounts as I present a brief history of Glasgow.

\section{Section 1.6 A brief history of Glasgow}

My first visit to Scotland occurred when I was in my late twenties. I had moved to the small community of Oban, on the West Coast of Scotland, to work at a summer job in a hotel. A beautiful area of the country, it is rather remote by Scottish standards as it is a three hour train ride from Glasgow. My introduction to Glasgow was first through the stories of my colleagues there who, despite the long journey, eagerly looked forward to week-ends in the city, known then for its vibrant club scene and excellent shopping. Glasgow was described to me as a fun place, even a little wild and rough around the edges. My own visits there were, at first, overwhelming, as I took in the city centre with its myriad of bars, cafes, clubs and restaurants and was met by out-going people who would often spontaneously pause in their own travels to ensure I was not lost. Little, however, was said of Glasgow's history. Years later, upon my return to Scotland, I resided in Edinburgh, only travelling to Glasgow for work. Interestingly, Edinburgh promotes its history from the moment you step onto the cobblestones. This may be the first of many contrasts that have been expressed to me by Scottish citizens from both cities and visitors alike. A discussion of these contrasts often includes the following: the atmosphere in Edinburgh is aloof, while Glasgow's is welcoming; Edinburgh is home to the elite, while Glasgow is home to the working class; and, Edinburgh's antiquity is ever present, while Glasgow's needs to be specifically sought to be found. I maintain that if Edinburgh is the political capital and head of Scotland, then Glasgow is its heart.

Glasgow is an old city, with ties back to the sixth century (Mozley 1977: vii). Although its original founding remains obscured, it has been related back to the "burial of the holy man 
Fergus in the sixth century by St. Kenitgern" (aka St. Mungo) (Ibid). Glasgow owes its development over the medieval period to the Christian church as it was originally the construction of the cathedral and the establishment of the burgh system that distinguished bishops as landowners (Maver 2000: 4). By the end of 1492, having established both a Cathedral and a University, Glasgow had become a "powerful academic and ecclesiastical centre rivalled only by St Andrews" (Glasgow City Council 2013: n.p.). The sixteenth, seventeenth and eighteenth centuries saw increased commercial expansion in the areas of linen, sugar and tobacco, and Glasgow earned a reputation as a beautiful city with an " attractive built environment" (Maver 2000: 25-26). The nineteenth century saw increased commercial expansion once again, with interest growing in coal and iron deposits in the West of the country, and manufacturing becoming a major focus for the city with the creation of a strong industrial base in the commodities mentioned above (Ibid: 37 ). This century also saw increased immigration into the city from the "Scottish highland and lowlands, accelerated by the clearances; from, Ireland, driven by the great famine of 1845-1847; and from Eastern Europe, whence Jews fled periodic pogroms", such that the population of the city rose to one million inhabitants in 1900 (O'Neill 2006: 30). O’Neill (Ibid) states that a "substantial middle class emerged, creating great wealth from manufacturing goods for the new world and the British Empire”. However, such a burgeoning of the population also put increased pressure on its people in the forms of overcrowding within the city, and disease outbreaks such as cholera and typhus which further exacerbated poor living conditions in the slum districts (Maver 2000:84-85). Glasgow also diversified its industrial base turning to "shipbuilding, locomotive construction, and engineering" over this time period (Glasgow City Council 2013: n.p.).

In the twentieth century, however, due to strike action and industrial decline between 1914 and 1922, Glasgow earned the reputation of "being one of the most fervently socialist cities 
in Britain" (Pacione 1995: 239). It was also during this period that the temperance movement took hold in Glasgow, and it has been "suggested that it was the earlier strength of the temperance movement that turned Glasgow pubs into the unsavoury drinking dens of the popular stereotype by pursuing municipal legislation that made it difficult for publicans to carry out improvements without having to pay for a new licence" (Ibid: 246). Sectarianism, a phenomenon that is responsible, in part, for helping to create a violent reputation of the city, is linked to historical persecution of Catholics by the early-modern Protestant state and, while it is tied to religion, it is also tied strongly to race (Ibid). As Catholics tended to be from Ireland originally, so were Protestants associated with being from Scotland (Bradley 2006). Despite the passing of decades, these 'racial' distinctions are still prevalent in Glasgow today, where sectarian divides are now aligned with football clubs such as those belonging to Rangers and Celtic.

It is during the twentieth century, as well, that Glasgow entered a period of severe industrial decline, the effects of which can still be felt today in certain neighbourhoods (Glasgow City Council 2013: n.p.). Travelling through the city today, one can see myriad displays of neighbourhoods that are run down and decrepit, with shopping precincts that cater exclusively to the budget shopper. I volunteered in one such neighbourhood, and was frequently warned about whom I could let into the building, and what approach I should take with people who appeared intoxicated or high. However, although these warnings did take place, they were partnered with statements that dangerous occurrences were rare. According to those who gave out the warnings, it was simply a matter of being street smart and paying attention. It is perhaps such openness to human frailties and social awareness that leads many to think that Glasgow is a hard city. How prevalent this reputation is became evident to me prior to leaving for Glasgow, when I had an opportunity to share my destination with some Canadian colleagues from my Master's program. Their immediate response was to draw back at the mention of Glasgow and utter statements such 
as, “Isn't it really dangerous there?”, or, “Are you afraid?" Conversely, many areas of Glasgow exude charm and offer a variety of shops and restaurants that cater to all socioeconomic levels. The people of Glasgow are warm, friendly and welcoming to strangers. I also found, through my own personal experiences with them, and through my research, that Glaswegians possess a wonderful, self-deprecating sense of humour, laughing easily at themselves and others.

The 1980s and 1990s saw a concentrated effort by the city to shed its violent image through social campaigns, festivals and a rejuvenation of the docksides that had fallen into disuse with the collapse of the shipping industry after World War II (Glasgow City Council 2013: n.p.). The year 1990 saw Glasgow winning the title of "European City of Culture" and, in 1999, it was designated the "UK City of Architecture and Design" both of which the city council claim have helped to further erode the negative image of the city (Ibid). The new millennium saw more rejuvenation of the harbour front and new, expansive housing regeneration schemes that can be seen in their completion in 2013. Currently, Glasgow is preparing to host the Commonwealth Games in 2014, and looking forward to the opening of a new entertainment centre that will draw more international talent to the city. Glasgow City is a mixture of architectural styles that range from the ultra modern to Gothic and Victorian design. Its streets are continuously thronged with people from all walks of life, always hurrying to and fro, and, as will be noted later in this thesis, socializing is the cornerstone of life. It is a city that appears to be ever pressing forward, towards an unknown, but, still highly anticipated future, despite the decline it experienced in the twentieth century. My experiences while living there in the summer of 2012, afforded me a personal experience of the welcoming and humourous nature of Glaswegians, some of which I hope is evident in the following chapters. 


\section{Section $1.7 \quad$ Introducing the Women}

This research would not have been possible without the generous contributions of time and energy of thirteen women living in Glasgow. These women came to me through a variety of channels and I feel, due to their contribution to this project, that it is appropriate to introduce them as I met and interviewed them. This serves the purpose of both contextualizing the encounters, and allows me the opportunity to share my first impressions and self-reflections that I tried to capture throughout my fieldwork. Again, as mentioned in the 'Scope and Limitations' section above some of the participants who are considered here worked with organizations that engaged in public health research or charities that had an alcohol focus. These participants, therefore, may have a heightened understanding of alcohol issues and women that may not be found in the general population. However, comparing the data obtained from the participants revealed common themes between those who worked in such organizations and those who did not. As already noted, I met with thirteen women in total, eight of whom were interviewed in two group interviews of four each, while the remaining five participated in individual interviews.

\section{Interview One: Joanne, Deirdre, Lila and Roxanne}

My first interview was the result of contact I had made with a non-profit organization in Glasgow prior to my arrival in June 2012. This organization was engaged in public health research, in particular, research that related to the causes of the 'Glasgow Effect'. Some of them also had experience with alcohol research, hence their interest in my research project. I had been in e-mail contact with several of the staff regarding health research in Glasgow, and found them to be encouraging and helpful as I prepared my move to Glasgow. My first interview took place in their offices, and was precipitated by circulating my recruitment poster to the staff of this organization by e-mail to see if any would be willing to meet with me for a group interview. This 
first interview served not only as a pilot for my questions, but also as an opportunity to conduct a group interview in an understanding environment. These offices are in a slightly older building in downtown Glasgow near the city centre, with friendly administrative staff who buzzed me in when I arrived at the door. The conference room where the interview took place was large with several tables aligned to create a large rectangle in the centre of the room. I had a few moments to contemplate the room and see if I could make the atmosphere more casual as the room felt quite formal. This I achieved by rearranging some tables into smaller formations for the group. I also brought along lunch items from a Marks and Spencer (M\&S) grocery store, as I had promised to deliver lunch for those who agreed to be interviewed in exchange for their time. I chose M\&S to supply the lunch because it is known as a 'high-end' grocery store, and I hoped that my choices would impress the interviewees and make me appear more professional. I hoped that sharing a meal would help put the group more at ease. With such considerations in mind, I also chose my clothing with care, choosing to dress in trousers and a sweater to mirror the 'smart/casual' attire that seemed to be standard for this environment, as I had observed on an earlier visit to the office. Again, I hoped that this would make me appear as akin to the interviewees and put them at ease quickly, while also conveying the message that I was a serious researcher. I cannot attest to how successful my attempts at appearing professional were received, but, in any case, my best efforts gave me more confidence.

In all four women participated in this interview. They were; Deirdre, a petite woman in her late twenties with shoulder-length dark hair, I knew from a previous meeting that she had a strong research interest in women and alcohol; Lila, a quiet and friendly woman, who appeared to be in her late thirties/early forties; Joanne who appeared to be in her late twenties/early thirties; and, Roxanne who also appeared to be in her late twenties. All participants seemed somewhat nervous so I did my best to explain consent and confidentiality to them, while also 
assuring them that they could withdraw from the process or leave questions unanswered if they so chose. We also agreed upon a time to finish the interview as they were meeting with me on their lunch hour. I promised to adhere to their timeframe.

This was my first interview so I was quite apprehensive, which I chose to share with the group. This seemed to have the effect of relaxing those present, as I believe it addressed the anxiety some of them had been feeling as well. Overall, I was pleased with my performance and felt I had addressed the group in such a way so that no one person dominated the proceedings. I was lucky to have mediation skills training in my background in this regard as it helped me interject at intervals to draw certain people out, or to remain silent when needed.

\section{Interview Two: Megan, Chloe, Jennifer and Mary}

My second group interview was arranged through a colleague from the same public health non-profit organization as those in the first interview. This woman responded to a general e-mail that had been sent out regarding a request for volunteers to the research study, and through her, I arranged to meet with several women at one of their homes located on the outskirts of Glasgow in a community I will call, Parceldon. I took the train in the evening from the West End of Glasgow and arrived about 20 minutes later. Again, I had brought some cakes from Marks \& Spencer, as compensation for the time these women were spending with me. Like many neighbourhoods in Glasgow, Parceldon has an inviting high street with several major retailers, and many detached and semi-detached houses in excellent condition. It feels very much like a North American suburb, and I later learned that it is considered by many to be a rather affluent area. This was reflected in the dress, appearance and accents of the women I met at the interview. Megan was a forty-something woman with dark hair and an athletic appearance. She was open and friendly, and gently teasing of the other participants. Another participant, Mary, was in her 
late twenties/early thirties, with a confident nature. Iris tended to speak a bit more quietly than the other women, presenting as the most nervous of them all, but was quick to relax once I began asking questions. Chloe has dark hair and, although born in England, appeared to be of Middle Eastern descent. Once or twice, when discussing questions relating to growing up, she spoke of her own 'culture'. This did not appear to refer to being Scottish, but in my novice state I forgot to ask the type of 'culture' to which she was referring in these statements.

I quickly realized that although these women knew each other, all four of them were not equally close as I did not sense the casual easiness that you find amongst close friends. I would say that while there might have been close relationships among some of the women one-on-one, as a group there was evidence of regular social posturing as is found in public, but not completely trusted, environments (a dinner party with strangers, for example). It felt more like people who knew each other only because of proximity and shared schooling of children rather than by choice. Saying this, there was much laughter and friendliness, despite the mild jitters I thought I sensed amongst the women. Whether that was due to my perceived superficial nature of their shared relationships or because the subject matter of alcohol consumption can be very personal, I remain unsure.

I am eternally grateful for my own and others' sense of humour in interview settings because I feel it immediately puts people at their ease and allows me to appear non-threatening. I am, of course, aware of the limits of humour, and refer here mostly to small comments that reflect the reality of my situation (how nervous I am, how much stuff I carry around with me to an interview, etc.), rather than artificial attempts to be funny. This interview lasted longer than the previous group interview and I felt good about the overall experience. I sensed in my questioning that while I was attempting to draw people out, I was also choosing not to probe too 
deeply in this group situation, as I felt a need to protect the participants within this environment. I did not want anyone to regret what they told me in front of others, so there was a limit to how far I would push in these circumstances. In individual interviews I felt more comfortable when people spoke freely and tended to probe for further details more readily.

\section{Interview three: Lena}

Lena responded to one of the recruitment posters that I had posted in public spaces around the neighbourhood and I arranged to meet her at a small cafe in the West End of Glasgow. She was not overly concerned about being overheard, and so was comfortable meeting in a public place. The chosen location was a crowded cafe with a casual environment where the seating areas were extremely close to each other. I had asked Lena to pick a place where she would be comfortable so was not concerned about the proximity of the other clientele. The overall atmosphere was casual and European. I loitered about outside for a while until a petite woman with blond hair looked up from where she was sitting on a stool and caught my eye. She approached me and confirmed who I was. I bought some coffee for us while she went back to her stool, and then joined her to get acquainted. Lena appeared to be in her late thirties/early forties and was dressed casually. She had a petite frame and spoke with a thick, European accent. I found out quickly that she was from Eastern Europe, but had been living in Glasgow for ten years. Despite the fact that she was not from Scotland, I felt it would be useful to conduct the interview to get an 'outsider's' view of drinking behaviours in Glasgow. Lena was friendly and personable, and stated that she found the way in which alcohol was used in Glasgow fascinating. I gave her a $£ 10$ gift card to a local retailer, Superdrug, to compensate her for her time as approved by my ethics board, and the interview began. Compared to the group interviews, this 
first individual interview was quite short, but it still provided me with some useable data and a chance to practice my interview skills.

\section{Interview Four: Bea}

I travelled to a working class neighbourhood in the south of Glasgow to meet with Bea. She was the friend of a staff member of the non-profit organization at which I volunteered during my time in Glasgow. In the course of the interview, I discovered that she worked with an alcohol-focused charity in Glasgow. The community where she resides was made up of a variety of tenements and shops that included takeaways, pubs, banks, convenience stores and supermarkets. It did not appear terribly affluent, and there were many shops and fast food takeaways that offered foreign cuisine. I met Bea at a coffee shop early one sunny Saturday morning in September and soon realized that she was a vibrant and no nonsense kind of person. She was in her forties, tall, with long black hair and warm eyes. I was a little nervous at first, as I sensed here was a fiercely independent and vocal woman, but that nervousness was soon assuaged by Bea's friendly manner and her directness in answering my questions. She shared many opinions and thoughts with me, and I felt the interview went well.

\section{Interview Five: Cilia}

Cilia appeared to be between the ages of thirty-five to forty. She was friendly and smart, with a quick wit. She obviously took great pride in being from Glasgow and was happy to explain or clarify certain 'cultural' concepts for me. She had been raised in a working class neighbourhood and was very forthcoming in her telling of anecdotes and experiences which might be considered 'typically Glaswegian'. Cilia was, like myself, a mature student. She was easy to talk to and, if anything, wanted to continue to talk even when we ran out of time due to a 
prior commitment on her part. The teahouse where we met was decorated in a modern style, and offered afternoon traditional teas. More expensive than the average coffee shop, it catered to an upmarket group of customers. After a brief chat to acquaint ourselves and order refreshments we started the interview. After the interview, Cilia asked me if I had noticed two people who had sat at the table adjacent to ours. I had only taken a cursory glance as I was focused on Cilia herself. She noted that they had seemed to be listening to our conversation and had appeared to grow more ill at ease the longer we continued. When I asked Cilia why this might be, she said she felt they were uncomfortable with her comparisons between drinking a bottle of wine at home and heavier drinking in pubs that we had previously discussed. She felt that her comments, stating that these two types of drinking were more or less the same, had made the couple uncomfortable. This did not bother Cilia, in fact, she seemed rather amused, and pointed out this situation to me in order to illustrate how opinions of alcohol use in Glasgow can vary depending on the type of drinking in which one engages. This interview was especially rich in detail, as Cilia has lived in the same neighbourhood for her entire life and is proud of her background and of Glasgow.

\section{Interview Six: Corinne}

I met Corinne while I was volunteering with a non-profit organization that provided those living in low-income housing with basic necessities (i.e. pots, dishes, glasses, linen etc.), which was located in an area of Glasgow that had a reputation as a rough neighbourhood. I worked alongside her one day within a shop and had seen her on various other occasions when I had other volunteer duties. I asked her if she would be willing to be interviewed and she agreed but asked if it could be done while we were volunteering in the shop, between customers. With her permission I checked out this arrangement with the Project Manager who was happy for us to proceed. Corinne appeared to be in her late fifties, petite with black hair and was born and raised 
in Glasgow. While working with her, I saw Corinne engage with people in a very kind and caring way, going out of her way sometimes to be courteous and giving to those who appeared vulnerable. However, she was also more than capable of taking care of herself when faced with intoxicated or customers who were high (a not uncommon occurrence in this neighbourhood), and was not afraid to speak up when needed. As well, Corinne had a dry wit and was selfdeprecating and life affirming at the same time. She was also very good at 'telling you like it is'. We met in one of the charity's shops that displayed local paintings and crafts, music, used furniture and vintage items. It was a bright environment with much activity, but there were always lulls between customers when Corinne and I could get together in the rear of the store and, while keeping an eye on the shop floor, we conducted the interview. Corinne did appear a little nervous before we began the interview, again I believe due to the nature of the topic. Despite a few pauses when customers entered the shop, the interview proceeded quite well. When it was over, Corinne appeared much relieved and surprised it had not been more uncomfortable.

\section{Interview Seven: Beth}

My final interview was with Beth, who was a member of staff with the same organization where I had met my fellow volunteer, Corinne (see above). She was in her late twenties and had a sunny disposition. Always pleasant and chatty, Beth naturally draws people to her. We met at the charity's headquarters one day after the office had closed and our meeting took place in the room where donated items were sorted and assembled. This was a room with a window that looked out onto the main office at the front of the building that faced on to the pavement. The room itself was full of shelving that held an array of linen, pots, pans, glasses, plates, toiletries, baby items, cutlery, Tupperware and crockery. There was also a large table where we went 
through donations to assess their usability. The room was rather dirty and felt more like a workshop than anything else. The heat was off at the time of our interview, so we kept our jackets on. As this was my final interview, and I had known Beth for several weeks through my volunteer work, the mood was light. Although I knew Beth better than the other participants, I was still not overly familiar with her views on the subjects that we discussed during the interview and had never socialized with her. This meant that the interview proceeded much as the others had done; with an initial nervousness and then a more relaxed conversation. Once completed, we locked up the shop and took the subway together to our respective stops.

All my interviews introduced me to dynamic women and allowed me to become more at ease with semi-structured interviewing. I found most of my questions were able to elicit useful responses, with one notable exception: that of questions related to women's health. I chose not to explicitly request information regarding the participants' knowledge of women's health as I was sensitive to posing questions that could be perceived as 'leading'. This made it somewhat more difficult for the participants to provide health related information easily. This will be discussed in greater detail in Chapter Four. The final section of this chapter provides a brief outline of this thesis, describing what will be discussed in the following chapters.

\section{Section 1.8 Summary \& Chapter Outline}

This chapter has provided the reader with an explanation of the research project, background to the research, its scope and limitations and the methods used during the study. It has also contextualized the material that will be presented in the following chapters by providing a brief history of Glasgow, and my first impressions of the women who volunteered to be interviewed for this study. Chapter Two provides a review of the relevant literature that provides the background to the research, as well as, theoretical considerations that are incorporated in the 
analysis. Chapter Three explores the three dominant themes of gender, class, and identity that arose from the thematic analysis. Chapter Four examines the links the participants identified between health and alcohol consumption, and considers the political economy in which this is situated. Chapter Five, the final chapter, provides a final discussion and analysis of the material, highlighting connections between the findings of this research study and the 'Glasgow Effect'. This final chapter also provides recommendations for further research and my final thoughts on the experience of 'doing anthropology'. 


\section{Chapter Two: Anthropology and the Study of Female Alcohol Use}

\section{Section 2.1 Introduction}

This project explores Glaswegian women's knowledge, attitudes, beliefs and practices of alcohol consumption from an anthropological perspective. This chapter provides a review of the literature that will help contextualize the material presented in subsequent chapters, revealing for the reader a deeper context in which the themes identified by the women in this study present themselves. The first section of this chapter examines the relationship the discipline of anthropology has had with alcohol studies and the emergence of women's alcohol consumption as a research topic. The second section explores the issue of gender, class, and identity, and their connections to alcohol use, highlighting relevant behaviours and belief systems. The subsequent section explores a critical medical anthropological perspective from which to analyze the issues of health, power, and political economy as they relate to issues of alcohol use within the Glaswegian context. The final section of this chapter provides a brief conclusion of the information presented.

\section{Section 2.2 Anthropology and A Woman's Place in Alcohol Studies}

Anthropology has had a lengthy and turbulent relationship with alcohol studies (Bennett \& Cook 1996; Heath 1987; Hunt \& Barker 2001). While alcohol has long been included in works by anthropologists, it has been criticized for its solely descriptive accounts of drinking practices or for treating alcohol studies as a "felicitous by-product" of other areas which are actively pursued (Hunt \& Barker 2001: 167). Alcohol studies within anthropology before the 1970s tended to overlook cultural theory and anthropologists failed to access material from other fields historically engaged in such work (Ibid). Further, Room (as cited in Hunt \& Barker 2001) 
accused anthropology of 'problem deflation', whereby anthropology's work on drinking tended to underemphasize problem areas with regard to alcohol consumption. Room attributed this to three main factors: firstly, the functionalist view common to anthropologists at the time, which maintained that societies had internal mechanisms that would revert the population to stability; secondly, he stated that anthropologists themselves came from backgrounds or "wet generations" which held personal, liberal attitudes towards alcohol consumption and, therefore, were applying this liberal perception of alcohol consumption to their work; and finally, anthropologists had too easily accepted the western cultural perspective of alcoholism as a disease and, as a result, were using the disease model as a means to assess the problematic nature of all alcohol related behaviours (Ibid; Room 1984). Those which did not fit this concept, Room (1984: 171-177) argued, were viewed as unproblematic.

Beginning in the mid-1980s, however, the trends began to change, marked by more engagement of anthropologists with interdisciplinary teams, an openness to mix quantitative and qualitative research methods and a new emphasis on cross-cultural studies (Hunt \& Barker 2001: 168-169). We have since seen an increase in the attention paid by anthropologists to alcohol studies. This may, in part, be due to three changes in anthropological focus including: the rise of medical anthropology as a sub-discipline; the more recent focus on the pathological aspects of alcohol; and the acceptance of a public health model by many anthropologists (Ibid). As well, as set out in the seminal work of David Mandelbaum (1965), the assertion that the relationships people have with alcohol are largely determined by culture, has since become a largely accepted theory (Gordon et al. 2012; Heath 1987; McDonald 1997). Anthropology has "underlined the wide range of cross-cultural realities involved in alcohol and its consumption" and how such realities are framed by culture (McDonald 1997: 11). Anthropology has also focused "on normal drinking and on alcohol as an integrated social artifact and culturally valued good" (Dietler 2006: 
230), in contrast to other disciplines which have focused on alcohol use as a social problem (Dietler 2006). Such a focus includes labelling alcohol as material culture, which can act as a "symbolic medium and social tool that are operative in the playing out of ritual and politics and in the construction of social and economic relations" (Ibid: 232). Lau-Barraco \& Collins' (2011: 48) study of social networks and alcohol use in non-student emerging adults notes that "perceived social norms for drinking represent our perceptions of the drinking habits and approval of alcohol use by others". In other words, we know that people around us ascribe meaning to the choices we make around alcohol that may reflect on other areas of our 'self' such as our moral fortitude, social class and self-worth. In a review of drinking cultures, Gordon et al. (2012: 3) found that sociocultural contexts had a "major influence on drinking cultures" and that there have been "dramatic changes over the past 30 years over how alcohol is viewed [and how] alcohol related policy and practice operates". They argue in their review that the previous categorization of cultures as either 'wet' or 'dry' drinking cultures is no longer valid as western drinking cultures are facing increasing levels of homogenization (Ibid: 7). How far this homogenization process has extended is difficult to ascertain as similarities are evident even between western and non-western drinking cultures. For example, gender roles are increasingly being recognized as a major influence on drinking behaviours and attitudes towards alcohol (Gordon et al. 2012; McDonald 1997; Thom 1997; Zilberman et al. 2008). We see cultural enactments of different gender roles with regard to alcohol, for example, where women are the brewers of alcohol while men are the consumers, or traditional circumstances where women serve alcohol to the men (Dietler 2013; Gose 1994; Hendry 1997; Holtzman, 2009). The issue of gender is discussed in-depth later in this chapter.

Women's drinking has previously received little attention, only becoming of increasing interest in recent decades. This may, in part, be due to women's changing social roles or the fact 
that the difference between the amount of alcohol consumed by women and men is decreasing (Lyons \& Willott 2008; Thom 1997; Zilberman et al. 2008). Studies of women and alcohol that do exist tend to concentrate on youth (i.e., those under 30 years of age) or college student-age women and their drinking behaviours and attitudes (Lyons \& Willott 2008; Rudolfsdottir \& Morgan 2009; Seaman \& Edgars 2012). Such a focus has left large gaps in our knowledge of women's drinking at various stages in their life cycles, in diverse socio-economic levels and of how alcohol use is framed within such parameters. Drinking patterns can have a deep impact on future health issues and, as such, these informational gaps need to be filled if we are to get a clear picture of the role of alcohol throughout a woman's life course.

Women's relationship with alcohol has been changing rapidly in the past few decades. The reasons for this are numerous although scholars and researchers appear to agree on the major influences which may have brought about these changes. McDonald (1997: 19) notes a historical cultural delineation in Western countries between men and women, where character traits have been attributed to men and women based on biological differences; examples include rationality versus irrationality, or men as powerful and women as vulnerable. It is, therefore, not surprising that alcohol behaviours are also framed within this gendered context. Thom (1997) notes that women's relationship with alcohol is not a new focal point for researchers but that this focus has, historically, been granted exposure and, then subsequently been dropped from the sight of public concern. For instance, in Victorian England, women's drinking was of major concern to the national interest of the country and was linked with a declining national population, high infant mortality and an unhealthy working class, all of which were believed to threaten England's supremacy as a world power (Ibid: 34-37). Jackson and Tinkler (2007: 253-254), in their comparison of 'ladettes' of the twentieth and twenty-first century and 'modern girls' of the 1920s, provide further support for Thom's assertion. 'Modern girls', or 'flappers', were “white, 
single, young urban women aged 16-30, usually upper or middle class” (Ibid). A 'ladette', though more loosely defined, is a woman who is a "pleasure seeker", who drinks excessively and appears to be "taking over the once male preserves of pubs and bars and competing with men unit for unit" (Ibid). Both of these categories seek to define young women who engage in alcohol consumption as troublesome and hedonistic, "driven largely by interests in partying and fun" (Ibid).

Similarly, since the feminist movement of the 1970s and its promotion of women's health as an important issue, the 'female alcoholic' has emerged once again in the United Kingdom and in the United States (Thom 1997). Thom (Ibid: 37-38) argues that the feminist movement was the driving force behind this renewed interest in women and alcohol, as women advocated for control of their own bodies and indicated a need for the medical field to respond to women based on their specific needs. She also notes three specific developments in the field of alcohol studies that allowed women and alcohol to become a problem worthy of attention within the traditional masculine spheres of medicine and science (Ibid). These were: a new 'interest constituency' of mainly female workers in the alcohol research and service provision fields who embraced feminist ideology; the emergence of new rationales to provide theoretical and scientific bases for addressing women's drinking as a separate issue; and the move from 'alcoholism' to the concept of 'problem drinking' (Ibid). Feminism can be seen as the first step towards the framing of a female relationship with alcohol, for example, over recent decades we have seen increased professionalization, social independence and financial freedom for women, which some have equated with the rise in female drinking (Day et al. 2004; Jackson \& Tinkler 2007; Lyons \& Willott 2008; Zilberman et al. 2004). Zilberman et al. (2004: 62) state that the entry of women into the job market, "and the narrowing of social roles likely altered the opportunities to drink and use drugs". Gender stereotypes had previously linked public drinking to masculinity, but as 
women began acquiring new social positions, entering domains formerly reserved for men, other cultural patterns, such as drinking behaviour, were impacted by this change (Emslie 2012; Lyons $\&$ Willott 2008; Plant 2008). The role of gender is one area that is of utmost importance to this thesis, but there are others which were identified by the women in this study. Therefore, the next section of this chapter discusses not only gender, but also class and identity and their links to female alcohol consumption.

\section{Section 2.3 Gender, Class and Identity}

Many authors note that gender is performative, that is, it is something we 'do' rather than something we 'are' (Butler 1988; Lyons \& Willott 2008; Ridgeway 2009). Gender has also come to be seen as a "multilevel structure, system or institution of social practices that involves mutually reinforcing processes at the institutional level, the interactional level, and the individual level" (Ridgeway 2009: 146). Paechter (2006: 14-15) explores the concept of performance of masculinities and femininities as arising from 'communities of practice', which include members who "have acquired an understanding of the world and ways of behaving [...] that is aligned with those of other group members". Such a viewpoint highlights that what it means to be feminine or masculine is ultimately understood as relational and, therefore, "constrained by the communities of masculinity and femininity practice" to which one belongs (Ibid). We enact and embody gender based on how gender types are perceived within our own culture (Rudolfsdottir \& Morgan 2009: 493). If we define gender as Lyons and Willott (2008: 695) do, as “a continually negotiated and tenuous identity achieved through repeated [and shared] practices", we can see how notions of gender fit with Bourdieu's (1990) habitus. The habitus is comprised of dispositions that are held and accessed by a group, and yet are flexible enough to respond to a variety of situations (Ibid 1990: 53). Alcohol use is one area where knowledge, behaviours and 
attitudes have a strong link to social constructions of gender, and can be seen as a way of “accomplishing gendered identity or 'doing gender' ” (Rudolfsdottir \& Morgan 2009: 493). In other words, practices and attitudes towards alcohol consumption are framed by our understanding of what is appropriate for each gender, and people are expected to perform in ways that align them with these gender norms. The following pages demonstrate connections between alcohol consumption and female gender expectations represented within the Glaswegian context.

Women's increased drinking has been met by what many authors call a 'moral panic' (Day et al. 2004; Lyons \& Willott 2008; Rolfe et al. 2009). Lyons \& Willott (2008: 694) note that changes in female drinking habits and patterns in recent decades have created this 'panic' "despite cross-cultural research showing that men still continue to drink more often and more heavily than women". This is due to a perceived threat to established gendered behaviours as women's social positions are changing. Drinking has traditionally been viewed as a male pastime, and social drinking spaces such as the pub have been dominated by men (Emslie et al. 2009a; Emslie \& Mitchell 2009b; Plant 1997; Plant 2008; Rolfe et al. 2009). Feminine ideals in western society have traditionally been attached to the role of women as mothers and nurturers and, as a result, have relegated women to the private, domestic sphere while men are seen as the 'natural' inhabitants of public spaces (Day et al. 2004; Jackson \& Tinkler 2007; Rolfe et al. 2009). Ridgeway (2009) explains how gender is one way in which we delineate the 'self' from the 'other', which can result in a system of binary opposition (i.e. men versus women) that people then use to make sense of their world. Similarly, women drinking for leisure in public areas that were previously viewed as male spaces present a challenge to the expected female gendered behaviour. Such a challenge can then be extrapolated to apply to other feminine norms and be perceived as a threat to the accepted dominant gender discourse. As a result, such 
behaviours are then targeted for criticism as they defy the cultural 'rules' as understood by a particular group (Ridgeway 2009: 151).

In the case of female alcohol consumption, such criticisms or sanctions are expressed through several prominent discourses. The first discourse is related to self-control. When women appear intoxicated in public spaces they are often labelled as 'out of control', whereas men are culturally sanctioned to appear in a state of inebriation (Lyons \& Willott 2008; Rudolfsdottir \& Morgan 2009). While women's drinking had previously been allocated to the home, the domestic sphere, women today are more likely to drink in public. Such "encroachment on traditionally male public space" results in what Rolfe et al. (2004: 327) call a negative "'backlash' of discourse and practice". This brings us to the next discourse that frames women as self-medicating and in need of a drink to "help them get through the day" (Ibid: 329). Such a view presents women as emotional and fragile creatures, as compared to their stronger male counterparts, thereby allocating women to the role of vulnerable subject (Ibid).

Women who drink alcohol in public are also often referred to as unrespectable, or promiscuous, to the extent that women can be labelled as sexual predators preying on men as the 'objects of desire' (Jackson \& Tinkler 2007: 257). Women with these attributes are not seen as empowered but, rather, as rejecting traditional female roles such as mother and wife (Ibid). That is, women are supposed to want to get married and become mothers, and those who do otherwise are seen as possessing low moral standards. There also exists a contrasting discourse, whereby women drinkers who appear as 'out-of-control' are seen as vulnerable to unwanted attention. Most troubling about this viewpoint is the related notion that should a woman who has been drinking be met with aggression or violence then she is somehow responsible due to the fact that she has been consuming alcohol (Day et al. 2004). Day et al. (Ibid: 175), in their analysis of 
United Kingdom (UK) newspaper coverage of women's drinking, noted that non-normative behaviour is often framed as partially to blame in the events leading up to aggression or attack on women. Women are, thusly, faced with two opposing identities: the predatory female versus the vulnerable female. In each instance, a threat is understood. For the former, a threat to men and their own gender role and, in the latter, a threat to women and self-recrimination for daring to act outside gendered expectations. This threat to men is further exacerbated by the fact that females traditionally have been expected to be able to temper men's aggressive natures and thereby, "discourage male-male violence" (Jackson \& Tinkler 2007: 260). Plant (2008: 156) states that, historically, women were the "informal social controllers of drinking and alcoholrelated behaviours". In such circumstances, it is not only women who are rendered vulnerable through non-normative behaviours, but men as well, for how will men react now that the staying hand of women is no longer available? Will they continue to drink to the extent that they could drink themselves to death? Rolfe et al. (2009: 332) state that as drinking is no longer under patriarchal control, women are now faced with heightened expectations to be self-regulating. This, they argue, is due to the existence of ideals of femininity and self-regulation that appear to run through both 'expert' and lay discourses of health (Ibid). This means that regardless of whether or not a woman feels these ideals are relevant to her own life she must act in accord with such ideals, as to do otherwise invites harsh criticism and ridicule. Similarly, even though women may be engaging in drinking in greater numbers and imbibing greater amounts of alcohol, this does not necessarily reflect a change in underlying notions of femininity and freedom that may be held by those drinking. For instance, when discussing patterns of behaviour shared by women drinking in groups, studies tend to support the idea that women believe they must look out for each other when in social situations involving alcohol consumption (Seaman \& Edgar 2012: 53-54). 
Female drinking is often framed as a discourse of equality and freedom (Holloway 2009; Lyons \& Willott 2008; Rolfe et al. 2009). Such a view maintains that women are being treated equally to men and sharing in similar experiences as they enter previously male-dominated spaces (i.e., pubs and bars) in order to engage in behaviours that are typically attributed to the male gender (i.e., drinking alcohol and socializing in pubs or bars, getting drunk, having fun, celebrating with alcohol). For example, Rudolfsdottir \& Morgan (2009), in their study of gender and young middle-class women and alcohol use in the UK, found that how women think about drinking reflects deeper attitudes about femininity. Overall, these women had a positive relationship with alcohol, one that they equated with fun and pleasure (Ibid: 498). Similar findings were found in other studies where participants linked drinking with nights out with friends and group identification and bonding (Lyons \& Willott 2008; Rudolfsdottir \& Morgan 2009; Seaman \& Edgar 2012). Women also noted that alcohol acted as a social lubricant in situations where people feel insecure or self-conscious, such as social gatherings and when dancing (Seaman \& Edgar 2012: 48). Despite these shared positive attributes of drinking alcohol, double standards of acceptable behaviour for both genders are well understood and represented in male and female perceptions of drinking behaviours. For example, Macdonald (1997: 135), in her discussion of drinking in the Scottish Highlands, notes that most public spaces where drinking occurs, such as the hotel bar, are seen as all-male spaces. When women do attend, it is infrequent and they tend to sit in all female groups (Ibid). Rudolfsdottir \& Morgan (2009: 502) claim that as women do not historically have "an established public drinking tradition upon which to draw $[\ldots]$ they are learning to create and negotiate their own drinking values and 'rules'", now that they have gained access to such places. Day et al. (2004: 167), in their discourse analysis of British newspaper coverage of women drinking, argue that "feminine ideals persist despite social and economic changes." 
Interestingly, women are among the harshest critics of those women they perceive as challenging accepted gender norms. Lyons and Willott (2008: 705) note that women can be much more critical than men of other women they believe are behaving in ways that appear to defy 'feminine' traits. This can be linked to the self-policing noted by Paechter (2006: 20) in her discussion of 'communities of practice'. She states that should members push the boundaries of gender norms too vigorously they risk finding themselves increasingly on the periphery of that group (Ibid: 14-15). There is a certain power and safety in being a member of a group and membership requires that norms are upheld and perpetuated by all members; it requires participation even in activities that are disempowering (Ibid). Such finger pointing may also serve an important function. By criticizing other women's behaviour, women are distancing themselves from that behaviour through a process of 'othering'. Emslie et al. (2012: 492), in their study of Scottish men's and women's drinking in early mid-life, explain how “heavydrinking older women position younger women drinking in public as problematic, [while] younger middle class women position women from lower social classes as vulgar, uncouth and excessive drinkers". In describing others in this way, these women are setting themselves in opposition to a problematic 'other'. They are therefore portrayed as behaving well when drinking alcohol while other women are simply out of control or morally inferior (Ibid).

As noted above, one way in which such 'othering' is achieved is by attributing specific drinking patterns to a particular social class. Young, middle class women in Emslie's et al. (2012) study compared their own behaviour to that of 'chavvy' drinkers. This reference refers to a recent phenomenon, the emergence of a new underclass in the United Kingdom known as the 'chavs'. Bennett (2013: 146), citing the Oxford English Dictionary, defines a 'chav' as “a young person of a type characterised by brash and loutish behaviour and the wearing of designer-style clothes ... usually with connotations of a low social status". The benefit of a label such as 
'chav' in a class system of inequality is in its applicability in a variety of contexts. That is, the flexibility of the term allows any person to find someone who is, relative to them, a 'chav', thereby validating his or her own, higher position within the class hierarchy (Ibid: 159). Hence, when the participants of Emslie's et al. study (2012) refer to 'chavvy' drinkers, they are attributing specific characteristics, in this case vulgarity and excessive drinking, as common to a particular social class.

Similarly, in their comparison of 'ladettes' and 'modern girls' of the 1920's, Jackson and Tinkler (2007: 255) note that ladettes are women who "are portrayed as being working or middle class". According to the Cambridge Advanced Learner's Dictionary \& Thesaurus ${ }^{5}$ (2013), a 'ladette' is defined as a 'a young woman who drinks a lot of alcohol, uses rude language, and behaves in a noisy way". However, a woman does not have to actually be a member of a working or middle class community to carry the social attributes that are associated with being a 'chav'. These characteristics include excessive drinking, being aggressive, behaving crudely and being sexually experienced, and are viewed as being the "“least desirable' [and] 'unrespectable' elements of working-class lifestyles" (Jackson \& Tinkler 2007: 255). Moreover, ladettes are also seen as behaving in masculine ways, thereby breaching dominant gender norms (Ibid: 262). The middle class have projected "the immoral, excessive and unrestrained onto the working classes, rendering them the 'Other' in relation to the well-mannered, restrained moralistic middle class 'Self”" (Rudolfsdottir \& Morgan 2009: 494). For the young men and women studied by Rudolfsdottir \& Morgan (Ibid: 500-502), women who were labelled as 'chavs' or 'ladettes' were seen as the "embodiment of bad taste", and as behaving in unfeminine ways. The participants judged this behaviour to be indicative of low morality, and thought that chav women were 'easy' sexually and did not know their place or their personal value (Ibid). The association here is clear;

${ }^{5}$ http://dictionary.cambridge.org/dictionary/british/ladette 
a lower socio-economic status (SES) is seen as determining a self-worth which is valued less than someone who comes from a higher SES.

Higher economic status has also been linked to semi-private to private drinking locations, as people with a higher SES are more likely than those from lower SES levels to drink in restaurants and at home (Holloway et al. 2009: 825). This is in contrast to the public drinking in pubs and nightclubs where 'chavs' and 'ladettes' are normally seen. Social class is inherent in some choices that women make regarding alcohol use, such as where to drink, and help to form or demarcate a particular sense of identity. The next section of this chapter explores the issue of identity further by examining how women make choices regarding their alcohol use based on the image they seek to convey to others and the connections this has to their lifecourse.

As we shall see in subsequent chapters, where one drinks is important in creating a particular sense of identity and can serve as an enactment of a coveted lifestyle. Similarly, what type of alcoholic drink one chooses also carries meaning. Holloway et al. (2009: 827) highlight that drinking wine with food is viewed as a 'cultured choice', whereas women drinking beer has been associated with lower social class behaviour (Lyons \& Willott 2008: 701). One such example concerns the attitudes around drinking beer in New Zealand and the 'acceptable' drinks for men and women (Ibid). In this example, most of the participants of the study equated women engaged in beer drinking as portraying a 'low class' social image, as compared to men who were supposed to drink beer whether it appealed to them or not (Ibid: 701). This same finding was mirrored in the work of Rudolfsdottir \& Morgan (2009: 501), where participants stated that the type of drink chosen could reflect identity type to others to the same degree as the type of clothes one wore or the accessories one chose. 
Issues of identity manifest in other ways when considered in relation to female alcohol consumption. Paechter (2006: 14) states that identities are relational and, as a result, they are flexible and multifaceted. Identities must be learned and enacted if one expects full membership within one's own community (Ibid: 17). This is evident when we consider the changes that occur over the lifecourse of a woman in relation to her alcohol consumption. When young, women may frame their alcohol use as emancipatory and indicative of an equal freedom to men. However, when women become mothers they are often expected to disengage from younger patterns of alcohol consumption in favour of the 'mother role'. Motherhood requires that a woman limit her alcohol intake not only during pregnancy but also once the child is born, in order to embrace a 'responsible approach' to alcohol where planning is favoured over spontaneity (Emslie et al. 2012: 486-493). This approach to drinking appears to fade as children grow up and become less reliant, and women once again have the opportunity, resources and time to create other identities for themselves (Ibid).

Rolfe et al. (2009:330-332) note how women also use alcohol to negotiate gender identities in three ways. The first of these is to adopt the role of 'one of the lads' so as to position oneself as manly and, thus, provide legitimacy in partaking in the male-dominated activity of public drinking (Ibid). The second requires that a woman behave in ways that others can identify as womanly and respectable, thereby protecting her moral identity in comparison to those women who present as 'one of the lads' (Ibid). The third way is through resistance of cultural norms altogether. This is achieved through identifying stigmatizing roles of women drinkers and refuting them (Ibid). Put simply, a woman will resist stereotypes by first labelling them, and then rejecting them as inapplicable in real life. Each of these strategies affords a woman a legitimate claim to public drinking spaces, while at the same time providing some protection against 
sanctions. Enactment of various identities is one way in which women are subverting restraints which are present in the patriarchal context of Western society.

\section{Section 2.4 A Critical Medical Anthropological Perspective}

Thus far this chapter has discussed themes relating to women's alcohol consumption and its connections to gender, class and identity. These themes will be explored further in Chapter Three. Ultimately, there emerges another key theme which ties together those mentioned thus far within this chapter. This is a theme of power and its inherent ties to gender relations, class consciousness, and the creation of identities. However, before exploring power further, I would first like to set out a perspective that is helpful in navigating the plethora of issues that arise when we seek to unpack women's alcohol use within the Glaswegian context. As mentioned in Chapter One, the interest of this project arose out of a larger research interest in the 'Glasgow Effect'. In relation to this, and to the links that can be made between alcohol use and health in general, I assert that no discussion of women and alcohol consumption can be said to be comprehensive without also considering the issues of women's health and well-being. As such, these issues will be discussed in Chapter Four. For now, I would like to provide the reader with a brief introduction to the theoretical perspective that will be used to explore the phenomenon of women's health within the context of this study. This will require a closer look at what it means when I say that I will be using critical medical anthropology and its links to political economy to frame relevant issues. The main components of such a theoretical standpoint will be presented in the following section of this chapter, and then demonstrated through their application to an overarching theme of this project: that of power and power relations in relation to alcohol consumption. 
Baer et al. (1986: 95) note that, in medical anthropology, a "critical approach must define health as access to and control over the basic material and non-material resources that sustain and promote life at a high level of satisfaction". Such a view would require consideration not only of those key areas that we normally think of when contemplating healthcare (i.e. doctors, nurses, hospitals, pharmaceuticals etc.), but also those areas that reside outside the healthcare system. As Baer et al. (Ibid) state, this means an "examination of contending forces in and out of the health arena that impinge on health and healing". Of particular interest here is the issue of power. More precisely, in order to apply a critical medical anthropological gaze, we must "understand health issues in light of the larger political and economic forces that pattern human relationships, shape social behaviour and condition collective experience, including forces of national, institutional and global scale" (Singer 1986: 128). Such forces include issues such as the capitalist world system, levels of social relations, governance, consumerism, and gender and class inequalities. In short, all those areas and influences that can be included within an examination of the political economy. The point that is of utmost importance here is to realize that issues of health and well-being do not exist in a social vacuum, but are formed and determined by the multitude of levels that interact to produce them. That is, one must consider what is happening in a local context not only from that micro level but also from the larger macro level of which it is a part. Forces beyond the local are impacting on what happens within our communities and require focused attention to determine how deeply their effects can be felt by individuals and communities.

Two examples will help to illustrate this concept. First, let us consider the Western biomedical health system that is present in the Glaswegian context. Such a system requires a multilayered system to respond to the needs of its populace. We, therefore, see a variety of stakeholders engaged in the health industry. These range from researchers, to doctors, to the 
allied health professionals, to management systems and government ministries and patients who actually require the health services on offer. This list, of course, is not exhaustive but provides enough detail for illustrative purposes. A critical medical anthropological perspective would require us to explore the larger corporations and those governments that control the supply of drugs and equipment, provide backing for hospital construction and growth, and dictate health policy (Singer 1986). Within the hospital infrastructure itself, we have a host of health professionals that range in authority, as well as, intricate bureaucracies that must be navigated to acquire care. All these players have a role in controlling access to care, who gets it and who does not, and also define 'illness' within a biomedical framework, that is, they medicalize social distress (Singer 1986; 2004). Within this multilayered system, a variety of discourses are given precedence. For example, such a system favours the biomedical model of disease which reduces illness to the acquisition of biological pathogens or to the failure of the body to function 'properly', thereby minimizing the role of social relations and their impacts on health. It also creates a hierarchical system of access to knowledge and care that "reproduces structures of class relations" (Singer 1986: 96). Thus, the class structure in which we live is replicated in our healthcare system. Traditional gender relations are also mirrored within this system, as can be seen by the traditional allocation of men to the role of physician and the women to the role of nurses, another manifestation of the gendered norms that relegate women to the role of carers and men to the role of decision makers (though, this is changing as women's social roles change). Finally, the treatment options and pharmaceutical drugs that are chosen for patients are determined by large corporations and capitalist forces of which we may not even be aware (Singer 1986; Singer 2004; Farmer 2004).

The second example relates to the importance of biomedical understandings of illness as touched on above. In this instance, Midanik \& Room (2005:1107) argue that as the "importance 
of biomedical factors increases, so does the focus on individualization of social problems". This means, that as we reduce our understanding of the cause of illness to biological factors, the less we are likely to examine social environments to assess what role they play in creating sickness (Ibid). It also means that we are more likely to blame individuals for their sickness, attributing its onset to unhealthy personal behaviours or wrongful beliefs related to health and wellness. For example, if we consider the dominant paradigm of alcoholism as a disease, this may lead us to think that the factors causing alcoholism are solely based on personal, biological factors. Social problems such as poverty, mental illness and disenfranchisement, therefore, go unconsidered. In the case of alcohol use, there would also be no consideration of the cultural factors which, as I have stated earlier in this chapter, are largely understood to define the acceptable use of alcohol. That is, what it means to be an alcoholic is not uniform across cultures and, therefore, other reasons can safely be assumed to impact on drinking patterns and behaviours. Associating illness solely with biological factors and an individual's actions might lead to blaming the victim for ill health, resulting in a disinclination to explore the cultural understandings of illness which also impact on states of wellness, as well as the power relations (e.g., gender inequality) that frame those cultural understandings.

As already stated, medical anthropology can be a useful approach in the study of relationships within society. Krumeich et al. (2001) demonstrate, through their discussion of health education and promotion, how anthropology can be of benefit when attempting to improve the health outcomes of communities. They explore how "contemporary approaches in medical anthropology study relationships between cultural and social structures, people's beliefs about cause, course, cure and prevention and their health behaviour" (Ibid: 122). They also note that the understanding of the physical body is diverse and socially constructed, which results in a social construction of masculinity and femininity that can "shed light on social differences 
between men and women" as I have already discussed within this chapter (Ibid: 123). Such understandings can then be linked to "illness representations and consequent health behaviours" (Ibid). This knowledge may be extremely useful in understanding how illness impacts on a person or a community and also provides direction when attempting to promote healthy behaviours.

A critical medical anthropological perspective can also be useful in exposing what Farmer (2004) calls 'structural violence'. By this he means the "social machinery of oppression" that is supported by all members of a society, enacted through "forms of desocialization" that serve to enable inequality, and yet appear to be "nobody's fault" (Ibid: 307). Examples of structural violence include, "epidemic disease, violations of human rights and genocide" (Ibid). Other examples included chronic poverty and generational unemployment, and alcohol and drug abuse which appear to be endemic to particular marginalized groups within society. In simple terms, structural violence are those supports, such as systems of belief, social enactments of oppression and created sentiments of disenfranchisement, that perpetuate a dominant order where social inequality is allowed to fester and grow. A critical medical anthropological perspective allows us to peel away the layers of society and the related culture, in order to disassemble the components that make up the social order, and to evaluate the factors that are perpetuating inequality.

Earlier in this chapter, we explored Ridgeway's (2009: 146) claim that gender can be seen as a "multilevel structure, system or institution of social practices that involves mutually reinforcing processes at the institutional level, the interactional level, and the individual level". Health and health experiences can also be viewed in this way. Ridgeway's (2009) multilevel analysis of gender follows Scheper-Hughes and Lock's $(1987 ; 1996)$ earlier questioning of 
Western and biomedical perceptions of the body, wherein they focus on meaning and analysis at the level of the individual; in the interplay between the sociocultural and 'natural' world; and, at the institutional level. Foucault (1978:140) addresses relations of power at the institutional level in his use of the concept of "bio-power", which he argues was a precursor to the capitalist system that manifested as "an explosion of numerous and diverse techniques for achieving the subjugation of bodies and the control of populations". This subjugation is achieved not only by limiting the choices available to a population, be it in healthcare, work opportunities, etc., but also by the creation of what comes to be considered 'accepted knowledge' within society. Similar to the 'communities of practice' as described below, belonging to a particular society requires that members share certain values and ideologies. These values and ideologies form dominant discourses that are shaped by the larger institutions within society and those who have the most power in relation to other citizens. These discourses become the benchmark against which acceptable behaviour can be judged, resulting in citizens who absorb and internalize particular concepts and behave accordingly, thereby, reinforcing the validity of such discourses. The citizenry, therefore, in adhering to the 'accepted knowledge' is, in fact, acquiescing to the power of others by conforming, through their actions and words, to the dominant discourses.

These three levels of analysis - individual, societal, and institutional - are examined more closely in the conclusion of this thesis, particularly in relation to the overall findings of this study and the relationship between the dominant themes that emerged. This consideration helps to capture the lived reality of Glaswegian women. Furthermore, it situates women's alcohol use in the contemporary context of Scotland's political economy. To end this section of the chapter, I now return to the overarching theme of gender and gendered power relations. The theme of power is one that binds these themes together, and they will now be explored from a critical medical anthropological perspective. 


\section{Section 2.5 Power and the Critical Medical Anthropological Gaze}

Paechter (2006: 15) states that there is "significant under theorizing of power" in consideration of communities of practice. She further states that such communities can have a "coercive conformity", in that the "taken for granted understandings operating within a community of practice can function as a repressive discourse that has significant effects on possible forms of identity" (Ibid). What is of interest to us here is how practices, attitudes, knowledge and beliefs about power are perceived, experienced and manifested in women's alcohol consumption and its connections to gender, class and identity formation.

The first, and most obvious, incidence of power that must be considered is that which exists in the relations between men and women. Rolfe et al. (2009:333) note that "the way women talk about drinking can be seen to reflect the complex and changing state of gender relations at the start of the twenty-first century, with women gaining equality but significant power imbalances remaining in place". Lyon and Willott (2008: 696) maintain that "gender is relational and power-based, and constructed through everyday social interactions". This is evident in the plethora of discourses that women must navigate in their attempts to enter a previously male dominated activity (i.e. drinking alcohol), performed in a male dominated space (i.e. pubs or bars). As already discussed in this chapter, these discourses include adherence to traditional gender norms and possible sanctions that may result when women attempt to challenge these norms. These sanctions come in the form of labelling women as vulnerable or predatory, out of control or unrespectable, or as possessing unfavourable attributes associated with lower social classes. Yet, despite the fact that women are choosing different ways to negotiate drinking encounters, their choices are ultimately tied to the fact that "women's drinking remains defined and measured according to men's drinking, and functions to reinforce the 'male 
gaze' on women's behaviour" (Ibid: 704). Women's drinking behaviours are consistently compared to those of men, and framed as either adhering to or challenging traditional female norms found within a male hegemonic society. Even when drinking is framed as an equality discourse, what we are actually witnessing is not the freedom to partake of drinking as an equal party, capable of behaving as one wishes but, instead, women frame equality as being able to act as men, that is, to behave as men behave. This is in stark contrast to men's behaviour which is seen to arise from the freedom to make whatever choices one wishes to make, not in comparison to women, but simply as free subjects. Lyons and Willott (2008: 708) argue that women who behave as men do when drinking are, in fact, "contesting their hegemonic subordination to men". It may also be that women simply do not have "an established public drinking tradition on which to draw", and have yet to create a new one all their own (Rudolfsdottir \& Morgan 2009: 502).

Women are also faced with excessive responsibility not only for themselves, but also for those around them. As previously discussed, this has been exemplified in how women's alcohol consumption is expected to change not only during pregnancy, but also as their children grow. This expectation of changing behaviours of alcohol consumption while children are growing up in the home has not historically been shared by men, and, I would argue, is not shared by most men in the present either. There is also the responsibility that women are expected to hold over men's behaviour. As mentioned earlier, women have been seen to have a duty to soothe men so that they do not engage in violent acts. Conversely, women also "have to be careful how they manage their own bodies, since in the heterosexual economy they are responsible for the desires they evoke in others and the consequences others' desires have for themselves" (Rudolfsdottir \& Morgan 2009: 502). This has been addressed above in discussion of situations when female victims of violence are taken less seriously by authorities if they had consumed alcohol before the altercation occurred. Similarly, women who are framed as predatory and sexually 
experienced are incongruent with the realities of public safety that still exist for women today (Day et al. 2004: 177). So, even when women adopt powerful positions they cannot escape real threats of physical harm they may face. Day et al. (2004:167-168), citing Cynthia Robbins and Elizabeth Stank, note that some feminist writers argue that "continuing views that certain activities such as drinking are 'unorthodox' for women can be regarded as perpetuating men's violence against them". This can perhaps be most succinctly illustrated if one considers how the media portrays women drinkers. Day et al. (2004:179), in their discourse analysis of UK newspaper coverage of women's drinking, found that what emerged was a "problematisation of women who consume alcohol, with particular reference to conventional discourses around femininity", most of which have already been discussed in this chapter. Media coverage of women's drinking also tends to be sensationalized and concentrated on 'binge drinking' of younger females (Holloway et al. 2009:828). Lyons et al. (2006) reviewed several popular UK magazines for alcohol advertisements targeted at men and women. They found that “representations of alcohol and drinking in women's magazines reinforce[d] particular forms of drinking (binge drinking, 'hardcore' drinking) and relate[d] them to the traditionally male world of professionalism, public sphere, adventure and risk" (Ibid: 231). Conversely, the alcohol advertisements in men's magazines used military and war imagery and text, and seemed to "function to provide men with a strong and secure version of masculinity", often relating masculinity to aggressive or violent behaviour (Ibid). The media's negative portrayal of women drinkers, whether in news items or advertising, reinforces biases and historically gendered behaviours that are currently widely accepted.

Powerful women are also accused of emasculating men, as though power is not an element that can be shared but, ultimately, one that can be held by only one gender at any given time. Day et al. (2004: 177) highlight that many studies have explored the concept of the 
'emasculating woman' and that "it seems to exert much fascination, even anxiety, for many men in contemporary society". I would add that powerful, or so called 'emasculating', women may provoke anxiety not only in men but in some women as well. In particular, this would include those who have obtained a high comfort level within the gendered environment discussed here and, therefore, have a vested interest in maintaining the status quo. I mention this as I feel it is important to remember that opponents competing for power within a patriarchal system are not delineated strictly along gender lines.

Lastly, it is important to highlight that women are gaining considerable power in one area related to alcohol consumption, that of gainful consumers within capitalist societies. I have already noted that women's drinking has increased in response to their changing socio-economic status, especially in relation to their newly acquired financial independence (Day et al. 2004; Jackson \& Tinkler 2007; Lyons \& Willott 2008; Zilberman et al. 2004). Holloway et al. (2009: 822) note that in urban areas "drinking in male-oriented haunts has been complemented ... by consumption in a diversity of hospitality establishments (e.g. licensed cafes, hybrid bar/restaurants), which have been self-consciously styled by their owners to challenge existing norms, and to be open to women". As well, 70\% of women over the course of the year purchase alcohol at the supermarket (Holloway et al. 2009: 822). Furthermore, women have emerged as a campaign target for the alcohol industry as, "alcohol is being advertised as a lifestyle product and alcoholic drinks have been designed, packaged and marketed so that [they] appeal to the female palette" (Rudolfsdottir \& Morgan 2009: 494). Producers of alcohol and entertainment venues have caught on to the power of the female dollar (or pound) and are doing their utmost to harness it. As women's alcohol use becomes more mainstream, it may be from within these responses to female consumerism that women start to create their own social identities related to alcohol rather than attempting to pre-empt those that have traditionally belonged to men. 


\section{Section 2.6 Summary \& Conclusion}

This chapter's review of the literature relevant to my research study explores common themes related to women's health and the consumption of alcohol. Gender, class, and identity emerge as important areas which are called upon by others when forming opinions of women based on their drinking practices. This chapter shows that women's drinking is tightly bound to issues of power and health within the Glaswegian context, as women's social, economic, and political power underpin their ability to assert their independence as adults who choose to consume alcohol in public and in private. Nonetheless, these social factors and systems of power may combine with alcohol consumption in ways that make women vulnerable to public ridicule, loss of social standing, and personal strife.

This chapter has also outlined the key points encompassed in a critical medical anthropological (CMA) perspective, and how CMA can be used to demonstrate the connections between power and female alcohol use. Lastly, the chapter introduced to the reader the political economic perspective that will be used throughout the rest of this thesis.

As shown in subsequent chapters, ill-health related to women's excessive alcohol use is but one aspect of the Glasgow Effect that this study attempts to address. First, though, let us turn to an exploration of the dominant themes by examining the words of the participants themselves as presented in the following two chapters. 


\section{Chapter Three: Women's Drinking and Sociocultural Factors}

\section{Section 3.1 Introduction}

Spend a little time in Glasgow and two things become increasingly apparent: the willingness of its citizens to engage you in spontaneous conversation, and the ubiquitousness of its pubs. Taken together, these two factors are a good representation of a key characteristic of Glaswegian life: sociality. While searching one day for a potential volunteer site, a charity shop in a working class neighbourhood, I happened upon one woman and stopped to ask her directions. Once she had ascertained where I wanted to go, she provided directions and then asked me what particular items I was looking for, as she could recommend much better places to get certain items at better prices. Similarly, at the 2012 World Piping championships, I shared a picnic table with a woman and her family. After exchanging basic pleasantries in which I explained that I was there to study mature women and alcohol use in Glasgow, the woman cried, “Don't ask me!" She then leaned in and whispered quietly, "I love it", before launching into a detailed account of how women drank back in her mother's day, and how it had changed over her own lifetime. Such examples are not anomalies, as the women I encountered on a day-to-day basis were always quick to acknowledge me, and then begin a conversation, sometimes of a rather personal nature.

The numerous pubs in Glasgow constantly appear to have customers, even if only a few 'regulars' in the middle of the day, and there are a variety of atmospheres to cater to a varied clientele. Pubs may be high end, posh establishments, neighbourhood haunts that welcome families, or 'old man' pubs that are dark and cater to a closed group of regulars. The importance of pubs is not only in their ability to serve alcohol, but also in their ability to provide an accessible locale where people, strangers or friends, can meet socially. In this way, Glasgow's pub culture is synonymous with community and conversation. 
These examples support a largely accepted theory in anthropology that the relationships people have with alcohol are largely determined by culture (Gordon et al. 2012; McDonald 1997; Room 2001). Anthropology has "underlined the wide range of cross-cultural realities involved in alcohol and its consumption" and how such realities are framed by culture (McDonald 1997: 11). In a review of drinking cultures, Gordon et al. (2012:3) found that sociocultural contexts had a "major influence on drinking cultures" and that there have been "dramatic changes over the past 30 years over how alcohol is viewed [and how] alcohol related policy and practice operates".

The purpose of this chapter is to explore three dominant discourses that become evident when conducting a thematic analysis of the interviews collected for this project. The first section provides a general overview of how the participants in this study framed alcohol use in Glasgow in general terms. The subsequent section explores gender differences found in the drinking behaviours of men and women, and how women's drinking behaviours have changed in recent years to become similar to men's. Following this discussion, special attention is paid to the role of aging in influencing drinking behaviours. The next section highlights issues of class and their relation to drinking behaviours within the Glaswegian context, while the succeeding section presents the argument that drinking and identity are closely linked. Lastly, the final section summarizes the findings presented in this chapter, and provides some final conclusions on the material presented therein.

\section{Section 3.2 Glasgow as a hard drinking place}

To ground and contextualize the discussions I had in my interviews, I always began with asking participants their overall opinion of drinking in Glasgow. Responses included not only the general feeling regarding drinking in Glasgow, but also, the overall view of women's drinking in Glasgow in the present. Drinking in Glasgow was frequently attributed to 'the 
culture', as many participants believe that there exists a drinking culture in Glasgow, and in Scotland as a whole, that is an intrinsic part of being Scottish. This was evident in comments made by women within this study as we shall see below.

Beth is in her late twenties, single, with no children and has lived in Glasgow for her entire life. Always pleasant and chatty, her sunny disposition naturally drew people to her. She was also a hard worker and took her work responsibilities seriously.

I think there's a lot of focus on alcohol. Everything tends to revolve around alcohol. If you want to do something it's, 'Let's go to the pub, let's sit in the house and get a drink.' Everything revolves around drinking. You get home and people say 'I just need to have a drink'. No particular group comes to mind. I know people from all backgrounds and ages. And I think most people I know drink more than I think is acceptable. (Beth, 09/27/12) Beth's comments are fairly typical of study participants. The general relationship with alcohol was described by study participants as very unhealthy, and many expressed the opinion that people in Scotland tended to drink too much. In this vein, drinking in Glasgow was described as a crutch and as a prop to get through stressful situations. In one instance it was referred to as 'a weapon'. Drink is seen to be at the centre of social life to the extent that a social occasion would not be complete unless one could have a drink. It is also seen as an extension of the Glasgow personality that seeks to be friendly and extends a sense of belonging to everyone. This sentiment was evident as Bea explained that being social was a key reason for her preference of going to the pub for a drink, rather than drinking at home.

So you know you're gonna get served, you're gonna get, you know, it's, it is a kind of feeling of belonging. I think that's very much the... I'm very much a pub girl, um, which is more than the drinking. I hardly ever drink at home. Um, but I'm, if I want a drink I want to go to the pub, because I like that social atmosphere, I like the people watching, I like just chatting to a blank stranger, you may never see this person again. But you can just, you know, have a casual chat.... (Bea, 09/22/12) 
For Bea, going to the pub is not only about having a drink but also about the people she may meet and socialize with within that environment.

The sense of belonging that many participants equated with drinking was exemplified in comments made by Cilia. Cilia noted that even in cases where people may have personal reasons for not drinking, such as religion, they may still be offered a drink at social gatherings. This is not ignorance on the part of the person offering the drink but, instead, is equated with respect and friendship.

Because it's just so socially normal to drink. Um, and I think as well if it's, if somebody's not drinking for religious reasons people may think that it's imposed upon you rather than of your own choice. Either they're giving you the opportunity to say that you're safe in this environment or they reject that imposed idea of not drinking. ... it's kind of a backhanded friendship. Actually, now, come to think of it, it's people trying to be friendly, trying to be nice. (Cilia, 09/27/12)

In this example, Cilia explains how offering a drink to someone who does not drink for religious reasons is a gesture of friendship and a way of telling that person that they are free to act as they choose within that particular context. The freedom to drink is framed in a liberal rights context, whereby the right to make a personal choice is important, and implies a rejection of the ability of religion to dictate behaviours, such as drinking alcohol.

Drinking was also seen as a reward for a tough week or as a way to relax, as explained by

Joanne:

Whereas now I would probably say that I probably do drink to relax, yeah, I would. And I would have a drink on my own now as well as with friends, so it's not just social. You know, it's like, a relaxing kind of 'rewardy' thing if I've had a hard, stressful week, I think, a glass of wine on Friday night. [Laughter and gestures or phrases of agreement from the rest of the group] $(08 / 30 / 12)$ 
Drinking has become so socially acceptable and expected, that to choose not to drink carries with it a variety of connotations. For example, many of the women in this study noted that if they said they weren't drinking they would be asked if they were pregnant.

Roxanne: That happens to you all the time! If you say 'No' to a drink everyone thinks you're pregnant.

Joanne: Particularly if you're married or if you had children already...

Roxanne: [Emphatically] All the time! There's like no other reason why you wouldn't have a drink. [Group laughs](08/30/12)

There was also a negative value judgement placed on those who didn't drink. Such people were seen as unusual, and not drinking was understood as carrying a strong stigma in Glasgow.

Roxanne: Think of all the people you know, everyone knows a lot of people, if you think of all the people, all the women that you know I only know one person that doesn't really drink very much, that's my age.

Joanne: Yeah, I would probably know one and, this is terrible, they're seen as being a bit odd. [Group laughs quietly]. I think they are odd anyway [laughter], yeah, but it's unusual to meet someone who doesn't drink. (08/30/12)

Corinne, as a non-drinker, was well aware of this stigma, as she relates when asked if her friends would share her views on alcohol.

Corinne: No. I think they are far more liberal in their views.

AJ (author): Would the general public agree with you?

Corinne: No. [Laughter] I think they would view me as a complete party pooper who doesn't know how to enjoy myself. [Mimics] 'Wouldn't go with her on a night out because she doesn't even drink'. (09/13/12)

Binge drinking was seen by all to be a consistent practice in young people's drinking patterns. The high levels of alcohol intake were said to have been normalized to the point that they were no longer noticed by most people and were deemed acceptable by many. However, 
many participants were also keen to point out that not everyone in Glasgow drinks, and, that Glasgow was not unique in its capacity to overindulge. Many felt that inhabitants of other areas in Britain were just as likely to drink to excess, yet, somehow, Glasgow was seen as the 'worst case scenario' by those living outside of the city.

\section{Section 3.3 Then and Now - the changing landscape of women's drinking}

What became evident from speaking with the participants of the study is the extent to which female drinking patterns in Glasgow have changed in the past 10-30 years. Many participants noted that women in the past would drink very little, if at all, and never frequented pubs of any kind. Reasons ascribed to this were threefold: economic; they simply had no extra income for this pursuit; normative, 'nice' women didn't drink; and, exclusionary. For this last reason, an example that was given was a particular type of pub that existed in the past. The 'spit and sawdust' pub was, historically, a drinking establishment that would contain a bar, a barman and sawdust on the floor. These men-only spaces were uncomfortable, very basic in furnishings and the sawdust was there to absorb any bodily fluids that might be forthcoming from the patrons. In short, a woman wouldn't want to go there and pub owners did not consider changing their surroundings to accommodate women's comfort. This example serves to illustrate the patriarchal nature of public drinking locations in the past, and the negative gender relations that discouraged women from frequenting pubs.

There was also the fact that in the past many pubs did not allow women and would not serve them if they entered. This also meant that basic facilities for women, such as toilets did not exist. Occasionally, there were side lounges in pubs where women would be segregated from the men and allowed to drink together. However, the pub would still regulate how much could be served to a woman as, while men were served pints, women would automatically be given half 
pints. This practice has died out in the past 5-10 years. The side lounges also offered women an opportunity to briefly enter, if only visually, the men's area of a pub. This was achieved through the use of a 'hatch' that had a panel that could be raised or pushed aside so that women, from their lounge bar vantage point, could keep an eye on their men in the next room. Of note here is that this behaviour, checking on one's spouse or family member, was itself highly gendered as men would not reciprocate through the use of the hatch to peer at the women.

Then the lounge culture began so that women could be in a separate room, with a hatch between the two rooms.... Women would look through the hatch from the lounge into the bar. (Mary, 09/09/12)

When discussing pubs that would have barred women in the past, Bea shared the following anecdote about one of the pubs in her neighbourhood:

... they actually didn't have a ladies loo until the '90s. And they were very much, um, again, that was before my time, before I was drinking in there but, um, yeah, that was very much a man's pub and although they allowed women in at that time I think, there had been a point when women weren't allowed in. Um, but they allowed women in but you had to go to the pub down the road if you wanted to go to the loo. They had an arrangement apparently with the next pub along that, um, yeah, women had to go there. (Bea, 09/22/12)

Drinking establishments such as pubs were seen as 'men-only' public spaces where men could socialize, while their wives were home looking after the home and the children. Women who attempted to enter such spaces were often considered to be either alcoholics or women of ill repute. There was an unspoken belief that 'nice girls' didn't drink, 'nice' relating to the moral character of a woman.

This gendered duality was also present in the drinks that were chosen by women and men and the number of drinks deemed acceptable for each gender in previous decades. Men would drink pints and spirits, while women would choose mixed drinks or fruit based alcohols such as cherry brandy; the emphasis being, socially at least, on the fruit rather than the alcohol. Some 
participants noted that whiskey was also allowed, provided it was good (i.e. expensive) whiskey and with limits strictly imposed on the quantity allowed. Participants noted that, in the past, when women did find themselves in circumstances where drinking was seen as acceptable, for example, at family events such as funerals, christenings or weddings, or dinner and dances with their husbands, the number of drinks permissible for women was very limited, ranging from 1-4 drinks over the course of the night. Both men and women would be watching all other women to ensure this social etiquette was not breached and women themselves would be acutely aware of what was expected. Should overindulgence result then the culpable party could expect censure in the form of gossip from neighbours the following day.

They would monitor themselves, it would be a self thing and it would be, you knew if you did that then the rest of them would all be going, 'Did you see the state of her last week-end?'(Bea, 09/22/12)

Participants noted that men could drink as much as they wanted and that, publicly at least, no one would be interested in their intake. Interestingly, no participants noted any form of private or public reprimands that would seek to address excessive drinking in males. The discussion presented thus far lends support to arguments presented in Chapter Two relating to drinking and gender. As previously noted, drinking has traditionally been viewed as a male pastime, and social drinking spaces such as the pub have been dominated by men (Emslie \& Mitchell 2009a; Plant 2008; Rolfe et al. 2009). Historically, when women entered these 'male' spaces they had to comply with certain expectations and perform specific drinking practices such as those described in this section in order to be allowed to remain within that public space.

Connectedly, Rolfe, Orford and Dalton (2009: 330) note in their analysis of women heavy drinkers" accounts that "the construction of drink as leisure is also often closely articulated with a 'gender equality' discourse". Similarly, some of the participants in this study were keen 
to link changes in women's drinking to feminism; however, this was seen in both a negative and positive light. Some noted that change began in the 1970s, a time when men could drink in pubs alone but women could not. From the 1980s onwards it was felt that women had become more empowered and confident and could therefore choose when, where, and what to drink. This view is mirrored in the literature where feminism can be seen as the first step towards the framing of a female relationship with alcohol and related factors which make this relationship even more visible (Thom 1997). For example, we have seen over recent decades more women entering the workforce which some have equated with the rise in female drinking (Zilberman et al. 2004: 62).

Gender stereotypes had previously linked drinking to masculinity, but as women are acquiring new social positions, entering domains formerly reserved for men, we are also seeing other cultural patterns, such as drinking behaviour, impacted by this change (Lyons \& Willott 2008). Women are also earning money independently, thus allowing them more control over how it is spent, and marrying later in life, with the result that women have more time and money to socialize freely. Some participants took pride in the fact that their own behaviours growing up may have played some small part in perpetuating gender equality in pubs.

Bea: Yes, it's now, whereas before it was very much if a man asked he got a pint, if a woman asked she got a half pint. The number of times I had to say ' No, it was a pint I wanted'. [Mimics barman]'Oh, oh, right.' [Self]'I didn't ask for a 1/2'. [Mimics barman] 'Oh, right, okay'.

AJ (author): And only in the past 5 or 6 years you say?

Bea: Yeah, I would say between, yeah, 5 to 10 years. There are now far more women drinking pints, and, I have to say, younger women as well. Um, and I must admit I look at that and I think well, I'm partly to, you know, I'm partly responsible for that because it's women like me, ya know...25-30 years ago that were going, ' No, I want a pint'. [Laughter] $(09 / 22 / 12)$ 
Conversely, one woman felt that feminism had not advanced women and their drinking freedom, but instead had led them astray.

We've won the battle of equality and gave up after it. You would have to become a man to be equal...Despite becoming far more educated or liberated we've failed to grasp the nettle and drank instead. All ages, it's becoming the norm I think for alcohol to be abused rather than enjoyed. (Corinne, 09/13/12)

Another participant noted that young women today had no idea that the freedom in drinking they have now had only come into existence in recent years and, therefore, did not consciously consider the same judgements that had been previously placed on women when drinking.

Bea: Yeah, I think there were women who just went "No, okay, I'm gonna get looked at for being in here on my own having a pint but I'm going to, why the hell shouldn't I?'

AJ (author): Yeah, and uh, do you think there's any of that with, I mean you say the younger women might drink pints now but do you think they go through that thought? Or does that occur to them?

Bea: I really don't think it occurs to them. Um, I mean I don't have a great deal of contact with young folk but I think it's now a case of, 'That's what I want, that's what I'm gonna have'.

AJ (author): Yeah, no hesitation.

Bea: No, I don't think you find 'What would people think of me?', whereas there was that, there definitely was that. $(09 / 22 / 12)$

Participants also stated that women are stronger now and more empowered, thus leading to more confidence to go out and enjoy themselves, as expressed by Mary and Megan in the following excerpt.

Mary: Generation behind us, women stayed at home and were quiet and men made the noise when they were out.

Megan: This [present] generation of women are happy to go out and make the noise. And consequently they drink. (09/09/12) 
Women who were interviewed also noted that in recent years, pubs had undergone a change in that they started serving food and the decor became more restaurant-like, resulting in more women in attendance.

Deirdre: I think why pubs have changed now from just being like a place where men went, like we went to the pub yesterday for lunch with work, like they wouldn't, there was no alcohol or anything but, just, that that's where we would go even if alcohol is part of it or not it...

Roxanne: Yeah, there's a whole pub/restaurant thing there that never used to be. $(08 / 30 / 12)$

These comments help illustrate how the pub industry is responding to women as consumers in recent years, attempting to render drinking establishments more attractive to women in the hopes of gaining their custom. This exemplifies the changing relations of power that we discussed in Chapter Two, as women are more consistently being targeted by the drinks industry as an attractive demographic with disposable income (Holloway et al. 2009: 822). This relates to the younger women in society, but these are not the only women who drink in Glasgow. The next section seeks to address how age and the stages of a woman's lifecourse can affect their drinking attitudes and practices.

\section{Section 3.4 Aging and its implication for women's drinking behaviours}

As introduced above, another factor which was noted by most participants as having an impact on the drinking behaviours of women was age. Participants noted marked differences between younger women's drinking behaviours and those of older women in Glasgow today.

I think it possibly depends on your age. I think younger people probably tend to binge drink a bit more than people round about my age which is kind of the mid-30s. (Lila, $08 / 30 / 12)$

When asked to summarize women's drinking in Glasgow, Bea had the following to say: 
Bea: It's a difficult one. Um, I think they're very similar now, similar to men's. I think that there's definitely much less of a difference.

AJ (author): Okay. Um, so would that include women in all age groups?

Bea: Pretty much, I think more so with the younger ones. Again, I think if you're, ya' know, into the, you know the older age there is still this hangover from the, you know again, my mother's generation even she will have a drink out but it will have lemonade in it, you know, it will have something. Um, to the extent that she will still just kind of frown at me drinking pints because she still has that 'Nice girls don't'. But, um, I think that as you get down to the younger ones and certainly what I've seen with young folk out in a, they're usually coming out as we're coming home on a Friday. You know, that's it, that's when you know, right, I'm officially middle aged, all the youngsters come out dressed for a night on the town at the point at which we're going 'I'm completely knackered I'm going home now'. [Laughter](09/22/12)

Participants noted that many young women were prone to binge drinking, which was defined as

drinking to the point where they were very drunk and unable to take care of themselves properly

in social situations.

What I notice more specifically is very young girls, um, their behaviour in the street. Having absolutely no regard to their well being. That's the thing that concerns me as a mother. My children, they see that as normal behaviour. To become absolutely so drunk you don't know what you're doing and it's become socially acceptable. And it is romanticized so much. You know, that, sitting your head down the toilet pan is considered to be a really cool thing...not. (Corinne, 09/13/12)

Though I suppose music festivals are a slightly different example 'cause they bring young people together. But I went to T in the park [a large local music festival for popular bands] here a few years ago, I was just like astounded by the level of people that were drunk and clearly weren't even there to listen to the music. Do you know what I mean? It was just about being drunk and drinking and didn't even know there was a concert going on. (Joanne, 08/30/12)

Some of the participants believed that the excessive drinking of the young women would eventually be curtailed if they choose to have children as priorities shift regarding how leisure time is spent.

Jennifer: I think it's your family; you use your days more. Back when you were a student you didn't care if you lost a day. 
Chloe: The week-end's quite precious isn't it, now? I suppose it's the whole family together in a way. $(09 / 09 / 12)$

Other explanations related to having children and getting married provided further reasoning as to why older women no longer drank or drank less, but, on closer inspection, these explanations were less about shifting priorities that can result from children and marriage, and more about the physical toll that drinking had on women as they aged.

I would drink less now because I couldn't drink as much. Like before I, before I had children and before I was married, I was going out at the weekends to the pub and to the dancing places and stuff and drinking for like hours at a time (group murmurs agreement). There's no way I could do that anymore, I'd be so ill. Because I suppose I had a big break from drinking when I was pregnant and then feeding a baby you obviously don't have drink for a long time and then so when you start again I would drink a fraction of what ... [I used to]. (Roxanne, 08/30/12)

Those who did not have children were viewed in a different light.

I have friends in their fifties who don't have children and they party harder than we did in our thirties, but then they will say on a night out that they actually would have preferred to have a family and have a focus to go home and behave. (Mary, 09/09/12)

Some noted that today people had more awareness of the damage that alcohol use could cause and, therefore, were more likely to begin to limit their intake; however, this was again linked to age and the physical changes that occur through aging that render hangovers more painful.

Yeah, I think people are more, again in my experience, I think people are more apt to say 'No, I'm alright, no, I've had enough. I don't want another drink', whereas before it would just be 'Och, to hell with it. I'll be fine.' Maybe also that's because my own social group, we're all getting a bit older and it's a case of it hurts more in the morning than it used to...[So] is it really worth the headache in the morning? (Bea, 09/22/12)

This could indicate that the desire to limit intake is not so much based on increased awareness of long term health damage, but due more to unpleasant short term outcomes of drinking in excess, such as hangovers that worsen with age. 
One participant noted that older women may drink just as excessively as younger women and yet, with experience, have learned how to mask their abuse.

\begin{abstract}
AJ (author): So you would say that older women don't drink the same way as younger women?
\end{abstract}

Corinne: Not as obviously. They learn a certain finesse in masquerading their abuse if it exists. $(09 / 13 / 12)$

Furthermore, some participants noted that alcohol abuse in older generations might be unintentional and occur as a result of confounding health messages in the media that had been misunderstood.

Roxanne:...I think there was sort of a health message for a while that sort of said if you're over a certain age and you drink one or two glasses of red wine it's good for your heart. I think that was just a really bad thing, because I genuinely think that a lot of kinda older people now who drink, like $\mathrm{X}$ was saying, wine with dinner whereas maybe they never used to when they were younger. I think some of those people now think 'Oh, it's quite good for you to have a glass of red wine'. But when you're having a glass of red wine at home you're not having a, you know, [and shows a small amount with fingers] but people are drinking like a bottle of red wine at a time. So I think there's, yeah, young people or younger people than us and maybe us drink too much but I think there's an older generation of people who probably drink way too much too but they're not falling about the street drunk, it's not that kind of drinking too much. It's just every day... [Group murmurs agreement].

Deidre: And because they might see red wine is like a good healthy drink and it's not what the people about the street are drinking.

Roxanne: Yeah, but it's still alcohol, and still doing the same thing to you. (08/30/12)

These discussions highlight that although young women are often accused of being the worst offenders when it comes to female excessive drinking, they are, in fact, not the only offenders. Such dialogue from the participants conveys an underlying belief that alcohol abuse is not only a problem among the younger population, but also among the older population. And, due to the maturity of older women (and men) and the manner in which they choose to drink, 
that is, not falling out of pubs but quietly, in their own homes, there might actually be harmful behaviour that is hidden from public view, and, as a result, absent from public consciousness.

This concept of 'hidden' drinking is one that was expressed by many of the participants in a general sense, and not only in connection with aging.

There's quite a strong drinking culture in [a working class community in Glasgow], um, and I would say that extends to hidden drinking culture where people have a bottle of wine every single night with their dinner. (Cilia, 09/27/12)

Others alluded to the amount of drinking that goes on 'behind closed doors' or at events where drinking may not be the main purpose of everyone meeting (for example, a book club) but where some women may drink too much nonetheless. These comments are notable in that they carry the weight of secretiveness with them. They can be seen as personal worries that are couched in vague terms so that they can be uttered publicly without threatening the privacy of the speaker, or may also mirror a cultural distrust of alcohol that is pervasive and felt as an undercurrent whenever drinking is mentioned.

\section{Section 3.5 Considerations of Class}

As already noted, when asked how they would describe the general relationship that people in Glasgow had with alcohol the dominant replies were: a poor relationship, alcohol was used as a crutch, and that alcohol was at the centre of the Glasgow social life. They also noted repeatedly that it was simply 'part of the culture'. Often, immediately after these initial replies many would state that they did not want to be seen as classist, but would then highlight the lower social classes as the worst offenders when it came to excessive alcohol use.

I prefer not to think in a class system but having experience being the age I am, I've got a broad spectrum and we still use the class system. It's definitely lower working class. (Corinne, 09/13/12) 
I feel that definitely depends on the kinda, the social demographic and who you are out with. Without putting towards a class system, if it's more working class the dependency is gonna be to go out to get drunk to give yourself a boost of esteem, to be allowed to go and dance and feel very awkward if someone walks into a room who are not drinking and enjoying themselves. Mary, 09/09/12)

'Working class' was defined as those who worked in low paid, manual labour, and who had little or no education besides regular school, which, in Scotland, can be legally abandoned at the age of 16 . Some stated that working class had once been used to differentiate between 'white' and 'blue' collar workers in which 'white' collar workers required some sort of professional qualification to work. To be working class also included a historical element as it would include a background where there had been no money, and the family would be very, very poor. Others described working class as those who accessed state benefits and lacked the basic life skills needed to succeed in society. The hesitancy and apologies of the participants in singling out lower, working class communities as the worst offenders for unhealthy drinking patterns in Glasgow presents another viewpoint that is commonly shared: a rejection of a class system. The participants know that a class system exists in Scotland, but are not comfortable with addressing or accepting it. Such a rejection is in juxtaposition to the English acceptance of (and some would say an adherence to) a class system.

And again it depends on your community and the background, and there's no way I can say this without sounding snobbish but it's also an intelligence thing. Um, and I [think] because of the clients we work with, we have a project that is specifically pregnancy and early years, and this is dealing with drugs and alcohol, drugs as well, drug and alcohol issues and they're pregnant or they have very small children and it's trying to educate them that one can't carry on doing this now because you are [responsible]. They will take the child off you if you do not behave responsibly and it's trying to get that education. (Bea, 09/22/12)

In the passage above Bea speaks of how a person chooses to drink as being an 'intelligence thing'. Her reference to 'community' and 'background' and her concern over 'sounding snobbish', demarcate for us that she is not actually talking about a person's individual 
intelligence or IQ. She is, in fact, addressing the lack of education among lower working class people in Glasgow. This link between class and drinking behaviours is further explored within the workplace as Chloe, who lives in an affluent area, describes a night out with workmates.

I work in Glasgow (i.e. the city centre) and when they, when we go on nights out in Glasgow, it's very much a binge. Just very much like, people bringing booze into the office before we've even got to the pub. ... [The location of her work means] that there is a mix of background. And I think because it's a mixture of ages and...a mixture of classes, so probably, maybe, more working class people but, or more working class backgrounds. (Chloe, 09/09/12)

Economic status and background therefore, are seen as having a direct correlation to a person's drinking behaviour. This appears to be a commonly held belief among participants in this study and one that they see mirrored in the media's treatment of stories related to alcohol. Not only is social class correlated to drinking behaviour, but also to perceptions of the acceptability of that behaviour.

A powerful example was provided by Bea regarding the abduction of Madeline McCann, a child who disappeared while on holiday with her family in the Algarve in 2007. She had been left sleeping with her sisters in their locked hotel room while her parents enjoyed a meal at a nearby tapas bar. ${ }^{6}$

Bea: I'll tell you a very good example again, it's not particularly a male/female thing but the Madeline McCann situation. Now, [the reaction was], isn't this horrendous, isn't this terrible? Now, that's because that's a middle class couple at a tapas bar, a wine bar, if that had been a [lower working class couple] down at a karaoke bar, swigging pints of Tennent's lager having left the kids, the papers would have absolutely torn them apart, completely castigated them. And to me, what is the difference? Parents left three very young children in a room on their own. Now, it doesn't, the way that society and the media portrayed that because they were a middle class, intelligent couple then probably it was totally different and I think there is still that if you want to take it into the wider...

AJ (author): Yeah, okay.

${ }^{6}$ www.theguardian.com/uk/madeleinemccann 
Bea: And that angered me very much because I thought if [they] had been [working class] they would have been ripped to pieces. Never mind getting a fund and money and all the rest of it. Whereas, all I saw, and I'm not a mother, I've got no maternal instincts at all, even I know you do not go and leave three children under five on their own. You just don't do it! (09/22/12)

Participants also noted that lower class drinking behaviours were not only the result of a lack of education and a historical background that rendered drinking more acceptable, but also that circumstances, which might account for the inability to rise in socio-economic status, might also provide reasons to drink.

Bea: Mental health. Economics, it can be very much at first, kill the pain, and it's an easy way, you know when things are going horribly, horribly wrong. I don't think that is exclusively to women, I think that's most people. Um, people who drink to enhance having a good time and people who drink because it's, 'I'm having a really, really shit time and temporarily I can forget about that cause I can artificially feel better.'

AJ (author): Okay. So when you say mental health is that what you mean?

Bea: Yeah, basically yeah, because if they're depressed or down. Yeah.

AJ (author): Okay, and [you mentioned] economics?

Bea: Economics, again if yer struggling, 'How am I going to pay the bills, how am I going to pay the ... It's people that are long-term unemployed, long-term, generationally long-term unemployed. Umm, there's no money, there's you know, poor intelligence, poor um, emotional kinda, and, you know, there's family breakdowns. (09/22/12)

Interestingly, the lower classes were not alone, as participants also noted that more affluent members of society, the upper class, also drank too much, they just had more expensive drink. I believe this supports the communal rejection of a class system mentioned earlier as participants are quite able to equate similar drinking behaviours across class distinctions. While they see that the lower working classes are receiving more attention for drunken behaviours, they are also aware that class distinctions do not negate the effects of unhealthy drinking. Quite the contrary, being rich just gives drinking a more attractive appearance. 
There's quite a strong drinking culture in [a neighbourhood in Glasgow], um, and I would say that extends to hidden drinking culture where people have a bottle of wine every single night with their dinner. Um, and it's kinda interesting that the people who have a bottle of wine every night with their dinner look down their nose at the people who go to the pub for a half an hour whose alcohol consumption might be considerably less.(Cilia, 09/27/12)

And I also think of higher, upper class who manage to perhaps not to become newsworthy as much and they're quiet abusers and victims.... More from the upper, not upper class but managerial levels. I can recall business managers trying to work and function absolutely honking with drink. And this was in a normal working day. Because taking clients out for lunch was normal so they all got stinking drunk. And so you had to deal with your boss being drunk when you were actually sober. (Corinne, 09/13/12)

And further:

Beth: ...You can have someone who is in poverty and drinks a lot. But I know people who are very, very well off and they drink the same amount, they just drink different brands.

AJ (author): And...does that make the drinking okay?

Beth: Not to me, but I can see how it can be viewed as that. I can see how if you are in your nice house with your expensive bottle of wine then it's grand and it's fun to be drunk and falling about. But if that person is living in poverty and running about the town with a four pound bottle of wine then people will look down on them because they're drunk. $(09 / 27 / 12)$

Interestingly, even though it may be cheaper to buy alcohol at off-licences (i.e. liquor stores) or supermarkets and drink at home, research shows that it is more affluent members of society that tend to regularly drink at home (Holloway et al. 2009: 824-827). This may be due to differences in drinking patterns. Lower classes have been noted to engage in binge drinking, that is instances where many units of alcohol are imbibed on one occasion, while those within higher socio-economic classes tend to drink more frequently, with some research finding that they tend to be heavier drinkers overall (Jarvinen 2012; Lahelma et al. 2010). This highlights the important class distinctions that are understood by members of the various echelons of society and also informs of differing class norms that affect drinking behaviours and attitudes. As the 
next few lines show, there are specific class norms that underpin attitudes on drinking and guide drinking behaviours and practices.

One participant spoke at length about the cultural ideal of being 'hard'. Briefly, being hard means that you don't show fear or sadness and are always ready to stick up for yourself if needed - either verbally or physically. This idea of 'hardness' was closely linked to working class areas of Glasgow and seemed to be targeted mainly at young men, although women were noted as 'being hard' as well.

Um....yeah, I think it is that thing of not being scared of violence and ready to use violence, you're ready to stand your ground, if not physically, than verbally. I think that is probably the best was of summing it up. To be able to hold your position verbally and put up with absolutely every single thing life throws at you, without resorting to help from somebody else, That's, I think, that's what 'hard' is... it's that giving cheek back to people, sometimes it can be quite good natured slagging [teasing] of each other and that Glasgow banter thing. (Cilia, 09/27/12)

'Being hard' then, is part of a collective identity that can be found in Glasgow's working class communities. This identity is also tied to a fortified wine called 'Buckfast'. This drink is known for its association to Non-Educated Delinquents (or NEDs) or 'chavs' who are generally thought to be young, working class people from disadvantaged areas who have created their own subculture within Scotland and elsewhere in the United Kingdom. This group has already been discussed in Chapter Two. Stereotypical behaviours that are associated with NEDs include "antisocial behaviour, and alcohol and drug abuse" as well as, "a lack of interest in education" (Smith 2012: n.p.). I say 'generally' as there has been some research that indicates that this subculture is no longer tied exclusively to the working class, but is also finding young, middle class people drawn to its ranks (Ibid). Such stereotypes in the media may help fuel ideas of Glasgow as a hotspot for excessive drinking. 


\section{Section 3.6 Drinking and Identity}

Past research has shown how drinking is related to the creation of identity amongst women. One example relates the attitudes around drinking beer in New Zealand and the ‘acceptable' drinks for males and females (Lyons \& Willott 2008). In this study, and as discussed in the previous section, most of the participants equated women engaging in beer drinking as portraying a 'low class' social image, as compared to men who were supposed to drink beer whether it appealed to them or not (Ibid: 701). A similar finding was mirrored in the work of Rudolfsdottir \& Morgan (2009: 501), when participants in their study stated that the type of drink chosen could reflect identity type to others, to the same degree as the type of clothes one wore or the accessories one chose. Similarly, women in Glasgow also use alcohol to create or enact certain identities. The first of these is closely linked with stereotypes related to excessive drinking.

And I think you know that, generally, Glasgow is seen as a hard drinking place and I think that that's kind of a stereotype. It's of course true of some people, but not completely. (Joanne, 08/30/12)

I think Scotland as a whole is perceived to be worse than anywhere else in the UK. But then Glasgow is the real center. (Roxanne, 08/30/12)

Such stereotypes of Glasgow do exist and are known by Glaswegians. Many feel that rather than dispelling the stereotypes, however, people living in Glasgow may actually take some pride in them and choose to live up to them.

I've travelled to Australia and been all over and get 'Oh, you're Scottish, you're from Glasgow. You must like a drink'. It must be that stereotype. The stereotype is that you're Scottish and then they find out that you're from Glasgow and then it's 'Oh, you're super Scottish!' [Laughter] 'You can really drink if you're from Glasgow.' Glasgow has a reputation you know that is next to none. Some people think it's quite a violent reputation, they're scared of it. But I'd say when we think of Glasgow there's lots of lovely things but definitely drinking is up there what would come to people's mind first. (Beth, 09/27/12) 
Um, I don't think, I honestly, hand on heart, don't think it's any worse than any other big city but I think that some people kind of like the stereotype, and like living up to it. You know? (Cilia, 09/27/12)

Many participants spoke of their belief that people learn about drinking and how to drink from 'their roots' and by seeing what others are doing around them as they grow up.

How do they form their opinions on it? Usually, I would say the majority would be first hand observation, family unit. (Corinne, 09/13/12)

And it's constantly all about you. I can remember as a kid actually being quite scared of the drunk people that were about in the street because even as a child you got that sense that they were going to be quite volatile about you. And I can see that, I've my own child as well, that he even although I'm trying not to be like my mom kinda probably put up with that fear 'just stay away from that man' whereas I try to be more relaxed about it 'That man's had a wee bit to drink and is being friendly to you so it's alright. Just be friendly back and it will all be fine'. (Cilia, 09/27/12)

And that's what they see their parents and everybody else round them, so kids come up with that it's, you know, that's what you do. (Bea, line 09/22/12)

The language used to describe different levels of drinking as discussed by the participants is telling as, although a woman may say that she 'doesn't drink', this does not necessarily mean that she never has an alcoholic beverage.

My auntie says she doesn't drink but it's not that she never, ever drinks. She might have a Bacardi Breezer or something in the house [unclear] but if we're at a social occasion she doesn't drink loads. And she would normally drive. Not drinking doesn't mean that she never, ever drinks. (Deirdre, 08/30/12)

Many participants noted a family member that emphatically 'did not drink', and then would go on to explain that one drink would be taken under special circumstances, such as Christmas or Hogmanay (New Year's Eve). This direct contradiction in terms was not noted by participants and would seem to imply that one drink taken at special occasions occurred in a liminal space outside the reality of day-to-day living, or, to put it more succinctly, day-to-day social drinking. Historically, a person would have at least one drink on special occasions, thus this practice is 
expected as an integral part of the tradition and, therefore, beyond the individual's choice. It is not 'drinking', per se, but 'tradition' and, therefore, permissible. This would suggest that the meaning of that 'one drink' on special occasions has a separate meaning from regular social drinking of the rest of the year. Such meaning is tied to other traditions that are still performed prior to special occasions. One example was provided by Cilia:

Because I know, even in my own family, we don't drink the rest of the year, but there's always something to drink at New Year because you had to welcome the New Year in and that's how you did it, was to have a drink. There were women frantically cleaning their houses for days beforehand because you have to have a clean house...I still feel the need to make sure, well, my bathroom's clean and my kitchen's clean and stuff like that for New Year, which is ridiculous because it's like a folk tale, a ridiculous superstition. You have to have the house clean, you have to have a window or a door open for the New Year to come in. (Laughter) (Cilia, 09/27/12)

Having an alcoholic drink to 'welcome the New Year' is simply another of a series of traditional expectations under these special circumstances.

Some participants noted influences of the temperance movement in Glasgow in the 1950s and 1960 s to explain that their families did not drink until their parents were much older, perhaps when their grandparents, the principal followers of the movement, had already passed away. There was also the impact of Glasgow's historically industrialized areas that were controlled by those financing business endeavours such as shipbuilding. Such control extended far beyond the shipyard to include appropriate lifestyles of the workers and their families (Damer 1990: 35-37). Finally, participants also noted that Glasgow was an increasingly multicultural city, and, therefore, drinking norms and behaviours were changing even in the present.

Drinking has also found a new connection to what my participants referred to as, 'lifestyle'. 
Roxanne: There's a whole lifestyle thing as well, isn't there? About going out, drinking, it's the whole 'Sex and the City' thing [all agree loudly] where you get dressed up, drink cocktails and pretend you live in New York. But you don't [all laugh-various pithy comments].

Joanne: And I think that's more for our generation than [all agree loudly]...

Roxanne: Yeah, you never saw the people in 'Sex and the City' rolling around drunk, that's not why they were going out drinking [all agree]. (08/30/12)

In this reference, women's drinking is tied to glamour and independence as exemplified in the HBO TV series, 'Sex and the City'. Pretending to 'live in New York' romanticizes women's drinking to make it more of an experience than a simple activity. Drinking, in this view, says something about a woman and her personality. She is not just drinking, but embodying the character of a cosmopolitan, fashion savvy, sophisticated woman.

Lifestyle also finds links to other social phenomena that have gained popularity in the recent past. Joanne discusses below how cooking shows on television and their rise in popularity has led her and her husband to incorporate drinking and meal preparation into a personal occasion to enact their bond as a couple.

I think, one other thing I was going to say, I think possibly, the whole like, drinking at home and kind of drinking too much as well is connected to the whole, you know, the popularity of food and good food and cooking, I mean every program, (group murmurs agreement) there are so many programs on now, like celebrity chefs and food or programs on restaurants. And most people I know my age really enjoy watching them. And most people I know enjoy cooking at home and stuff. Whereas my parents' generation that would never have happened, you know, having a glass of wine while they cook and cooking together that never would have happened. [Group all laughs]. Whereas now that's very, you know people really enjoy doing that, and it's really, not the thing to do, but you know it's a popular thing [Group murmurs agreement]. (Joanne, 08/30/12).

Roxanne expands on this theme, tying cooking at home to the image it evokes in the minds of some women. 
It's an aspirational thing, isn't it? To have that that whole, like open plan kitchen and dining thing and chat to people while you make dinner and all drink wine. (Roxanne, $08 / 30 / 12$ )

Attributing meanings such as affluence, sophistication, living well, and up-market methods of socializing with alcohol render drinking more socially acceptable. It may be this reason why, as discussed in previous pages, we see more affluent members of society drinking at home as these are characteristics that are coveted by this level of society. It links drinking to character traits that are seen as desirable by women, and also links alcohol use to larger western, social discourses that equate personal worth with financial success. Such meanings endow drinking with particular gender values, thereby changing it from an activity that has historically been male-dominated and performed outside the home, to one that invites others into a traditionally female space, the home. This also serves to make those invited to such events feel more cultured and superior to those who cannot afford to, or who simply do not, socialize in this special way.

The preceding examples show how the manner and location in which a person drinks in Glasgow have a direct link to how people want to see themselves. Drinking specific types of alcohol in particular environments, therefore, is actually a public statement that reflects a person's class and seeks to create a particular identity that can be witnessed by others. The decision to drink a particular way is not innocuous, but a deliberate 'identity-forming' activity.

\section{Section 3.7 Summary \& Conclusion}

This chapter has explored drinking attitudes and behaviours, both past and present, in Glasgow. We have seen that a variety of cultural factors affect how drinking occurs, and, that drinking behaviours and patterns are endowed with meanings that reflect certain cultural characteristics that are well understood by Glaswegians. The participants have voiced concern, as well as a playful, social attitude towards drinking, and witnessed changes in women's drinking in 
recent decades as a sign of the changing place of women within society. In the Glaswegian context, drinking is strongly tied to gender, class, age, and identity, and enacted in ways that convey information and messages to those sharing the same public space. Drinking is not only an individual choice, but a very public statement. Such public statements are not unconscious actions but are actively chosen by those who employ them, and are important in communicating a sense of self to others.

The next chapter elaborates further on these themes by exploring the larger political economy in which drinking in Glasgow occurs. This requires a closer look at the role of health and its importance in affecting drinking behaviours in women as articulated by the participants themselves; at Glasgow's attempts at controlling drinking behaviours; at Scotland's place in connection to the European Union; and, finally, an exploration of the media' s portrayal of drinking in Glasgow. 


\section{Chapter Four: Health, Alcohol and Political Economy in Scotland}

\section{Section 4.1 Introduction}

While living in Glasgow I was struck by the number of women who were open about issues that were affecting their physical and emotional health. At one cafe I frequented, I learned of the problems that one of the older servers was having with her feet, how much they hurt after work, and I was invited to view the slippers that she had to wear when working if she was going to be at all mobile in her job. Another woman, knowing I was there for alcohol research, made a point of telling me that she had consciously chosen to use alcohol as a coping mechanism during a time of marital strife, purchasing a bottle of wine each night, or inviting friends around for dinner and drinks (with the emphasis for her on the drinks) so that she would not be alone. These two simple examples serve to demonstrate spontaneous admissions about personal health and wellness that spring from the women living in Glasgow and their desire to share these situations with others. Connectedly, any comprehensive discussion about alcohol use must also include an examination of the impact it may have on different spheres of a person's life. Of particular interest to me was the affect that alcohol use had, if any, on women's overall health, and how connections between alcohol use and its effects on daily living were conceptualized by the women in this study.

While the previous chapter explored the dominant themes of gender, class and identity that arose from the collected data, this chapter concentrates on various sub-themes that highlight the connections that the women make between alcohol use and how it affects their everyday lives. It also explores links within the political economy in Scotland in order to further contextualize this subject for the reader. Such an analysis provides a more holistic view of alcohol use in Glasgow, by identifying factors on a macro level that also need to be considered 
when studying this topic. To this end, the following section explores what the participants of this study consider to be the impacts of alcohol use on the lives of women in Glasgow. In particular, I will demonstrate how the women in this study frame connections between alcohol use and women's health. The subsequent section examines if participants believe they can access information on alcohol consumption and how this information is perceived. Following this, the chapter builds on the previous two sections by investigating issues of the accessibility of alcohol and how women conceptualize healthy levels of drinking. The next section contextualizes the topics covered in this thesis, thus far, through an exploration of alcohol's place within the political economy of Scotland. Lastly, the final section summarizes the chapter and provides a brief conclusion.

\section{Section 4.2 Alcohol Use and Women's Health}

As stated in the introduction to this thesis, the interest for this research project found its roots in the phenomenon known as the 'Glasgow Effect', the relatively recent phenomenon that Scotland (and Glasgow, in particular) is the region with the highest mortality rates in Western Europe (Walsh, D. et al. 2010b). One question generated for the project was whether or not women's alcohol use may be a contributing factor to these poor health outcomes. To answer this question, further attention must be paid to how participants from this study understood the impacts of alcohol use on their health, and whether or not this emerged as an area of concern for them within the interviews. As I have stated, my primary interest was how alcohol use or abuse impacts directly on women's health in Glasgow; however, to avoid leading the participants' responses I declined to pose the question delineating health as my main concern. Instead, I phrased the question as openly as possible: What do you know about alcohol's impact on women? Many participants asked me to clarify, confused by this question and wondering what 
sort of information I was looking for. I endeavoured to be as vague as possible even in my attempts at clarification, as I hoped to elicit their genuine reactions. The results of these discussions are presented in the next few pages.

When asked directly about alcohol's impact on women, one participant expressed how she felt when seeing women who drank excessively:

I really hate to see a drunk girl. I get frightened for her. I think there is a vulnerability, absolutely. I've seen some drunk girls turn really, really nasty when they've been drinking as well. Absolutely horrible, but I think it is more the vulnerability that scares me. I've had to go out and get a couple of my friends and like, 'okay, you're coming home now'. And they get to that point where they need to be looked after and that's horrible because I think I am a strong female character, I don't like feeling like I could ever feel that vulnerable. (Beth, 09/27/12)

Other participants echoed Beth's concern over women's vulnerability, by citing ties between alcohol use and poor decision-making. During one group interview, fellow participants were quick to commiserate with Deirdre regarding feelings elicited the day following a drunken night out.

I think you can feel guilty afterwards if you don't remember what you've done or you might've fallen out with someone or had a tiff with them ... but [it] didn't really have to happen but because you were both drunk... [All present shudder and mention the word 'guilt' at various times laughing sheepishly together]. (Deirdre, 08/30/12)

Later, the same group discussed how they often took less care regarding personal safety than was probably prudent when on a night out.

Joanne: So in terms of your personal safety and stuff, you know, I think sometimes it probably does put you in a more compromising position. [All murmur agreement].

Roxanne: I've walked home from a lot of places that I wouldn't have walked home from if I hadn't had anything to drink.

Joanne: You're just not thinking logically...

Deirdre: But it's also like you can't be bothered to be hanging around for a taxi... 
Roxanne: You just want to go home... (08/30/12)

Interestingly, such reflections on poor decision-making had a gendered perspective.

I think that's probably the same for guys as well as girls, probably [all murmur agreement]. I think some girls maybe would do things that they wouldn't have done maybe and they might regret it. And, I'm mainly talking about kind of sexual type things, whereas, I don't know, guys might do it anyway whether they're drunk or not and not be bothered. Whereas I think sometimes girls may do things maybe where they then think "I wish I hadn't done that", or whatever so I think, I do think that's maybe slightly different for girls than guys. (Joanne, 08/30/12)

Joanne explains that women, when drinking excessively, run the risk of performing sexually in a way that they might not consider when sober and that such activity would be a source of regret for a woman. Conversely, she also states that similar sexual activity performed by males under the same circumstances are not blamed on alcohol use alone as, "guys might do it anyway whether they're drunk or not and not be bothered". Such a view highlights a gendered view of sexuality and acceptable sexual behaviour. The above comments indicate a belief that women need to take more care when choosing sexual activity as it may be something that will be regretted later, whereas men can act with impunity. This regret stems from choosing sexual activity when personal judgement is impaired, as the choice would have been different if the situation had happened when sober.

The regret discussed by the women in this study also reflects their understanding of the common judgements of drunken women's behaviour by others that we have previously discussed. These include two extremes of a broad spectrum of sexuality: a drunken woman is often labelled as either promiscuous, out-of-control or as a sexual predator. Both of these carry with them a judgement of low morality or of an inability to care for oneself. Similar value laden judgements are not often applied to men. Tellingly, participants noted that the woman herself may regret the sexual behaviour on a personal level, that is, she may feel that had the alcohol not 
been involved she would have behaved differently, but no one mentioned that others may censure the woman for partaking in such activity. That is, others were not noted to engage in shaming behaviour. This is not to say that such behaviour never happens but, rather reflects the assumption that personal nature plays a role in the choice of sexual behaviour, and highlights the self-responsibility that women maintain in this context. A woman will simply know when she has behaved in a way that will be viewed by others as inappropriate and will censure herself. The inappropriateness of the behaviour is linked to the fact that a woman loses control of herself, and, as a result, engages in sexual activity that is unsafe and deemed to reflect a lack of selfrespect, thereby reducing the moral value of the woman. This demonstrates how engrained such notions of sexuality are, as they are self-enforcing and do not require an external voice to judge when behaviour is appropriate or not.

The 'vulnerability' cited by the women also reveals that the first health impact related to alcohol use with which they identify is emotional health. They explore the negative mental and emotional impacts that can result from drinking, as they link it to personal safety and making poor choices. None of the participants mentioned regrettable sexual encounters as forced encounters, and while they implied that they had sometimes put their personal safety at risk, this presented as a reduced capacity to care for oneself. This alcohol-induced incapacity put them at risk of outside threats or led them to put themselves in unsafe situations (e.g. walking home instead of getting a taxi late at night). The spontaneous physical reaction of participants when discussing the impacts of alcohol use on women (i.e. shuddering, shaking their heads, murmuring agreement and similar words such as 'guilt') demonstrates how quickly such drunken encounters can be recalled, and that most of the women present genuinely share these common experiences. 


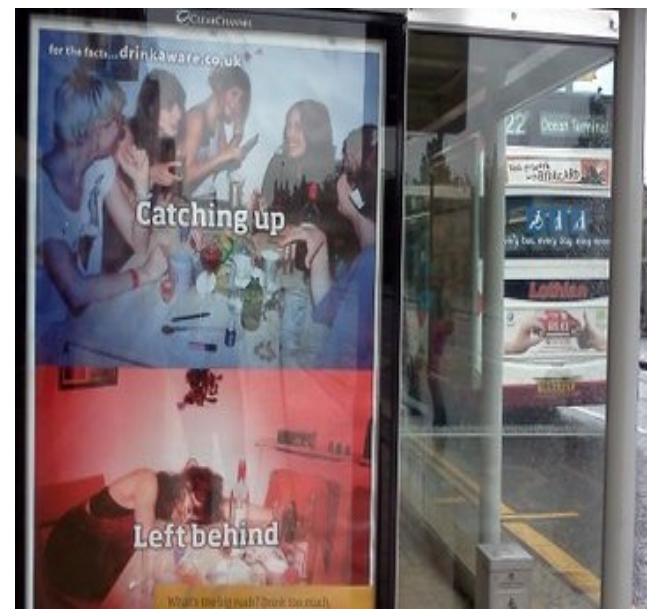

Figure 1 An alcohol awareness poster on a bus shelter in Glasgow targeting female drinking patterns

Other participants articulated ways in which alcohol directly affected women's physical health.

I think it's becoming worse for women, physiologically. We're climbing up there with the guys for liver disease. (Corinne, 09/09/12)

Not much, all that I can imagine, I guess. That's only..., very bad for the liver, obviously, and the circulation. Especially somebody who is not doing exercise, it's even worse. Because, they go after, let's say talking about the [binge drinkers], they always end up in a late night, like a chip shop, and all these kinds of fatty, greasy foods. So, I think it's almost necessary after a .... It's related... (Lena, 09/12/12)

Corinne states that women are 'catching up' with men when it comes to health problems, from which one might deduce that women have, in the past, not been known to suffer negative health consequences from alcohol use. Lena can not only express how alcohol use affects physical health, as she notes it is, 'bad for the liver and the circulation', but also ties drinking to another high risk health behaviour, poor eating habits. In her opinion, excessive drinking is directly tied to consuming 'fatty, greasy foods,' which also has an impact on women's health over time. However, in most cases, the participants did not elaborate on these health effects unless it was to emphasize the impact of alcohol on women in general:

I think it's another, the way it's [alcohol] used. It's another nail in our coffin that we could do without, particularly within the west of Scotland. (Corinne, 09/13/12) 
Another health related concern that was mentioned by one woman in this study was drinking alcohol while pregnant.

[There are] still too many pregnant women drinking...heavily. (Mary, 09/09/12)

Correspondingly, Cilia commented on the issue of how alcohol and pregnancy were handled in social situations.

Cilia: Even when I was pregnant you'd get people doing that. 'Are you really sure you don't want a drink? You'll be okay... How many months are you? Okay, just wanted to ask'.

AJ (Author): So would that be okay for most people, to drink when they were pregnant?

Cilia: Yeah, you would just, other people would go, 'Och, just one'. They'd be okay with just one. That kind of thing. Obviously not the first wee bit, but then you tend to not know in the first wee bit, but then when you're starting to get towards the end of term people do start 'Ah, just go on, just the one.' And you're like, noooo.

AJ (Author): So, is it okay to have, is there a limit, you know, to what you can have if you're pregnant? So you're saying you wouldn't really in the first bit, they wouldn't offer but they would towards the end.

Cilia: Yeah, I think so. People will still say to you 'Sure?'. Some people would be okay with it, understand that you're pregnant and that's not a good thing to do, other people would still be 'Are you sure? Do you want a drink?'

AJ (Author): So there's no fear about having a drink?

Cilia: Some of the people would and some people wouldn't. It just depends what their general attitude to drinking is. $(09 / 27 / 12)$

Holloway et al. (2009: 822) note that “biomedical interest in women’s drinking during pregnancy dwarfs all other aspects of research," stating that this reflects the hegemonic discourse in Western society that frames females as child-bearers. Similarly, Day et al. (2004: 173) highlight that "the effects of alcohol consumption upon female fertility and unborn children" tend to dominate health warnings regarding female alcohol consumption in the media texts that they studied. It is surprising, then, that more women from this study did not mention ties between 
female alcohol consumption and pregnancy, given that we have seen that female drinking is often tied to motherhood and, as previously discussed, nationhood. Such an omission is even more unexpected when we consider that out of the thirteen participants, ten had children. Perhaps this is due to the way female social drinking is handled in Glasgow as articulated by Cilia.

Personal choice dictates what level of drinking is, or is not, acceptable to a pregnant woman and, therefore, is not yet a fixed expectation within society. Coming from Canada, I was struck by the difference in the vehemence between the Canadian view of not drinking when pregnant, and that which I experienced in Glasgow. For instance, in Canada signs noting health warnings about drinking when pregnant are displayed prominently in bars and restaurants. I saw no such displays in similar venues in Glasgow. This may be due to the personal nature of such a choice, as discussed above, and also the weight given to personal experience versus 'expert' opinion within this context as we see in the following example.

Women in this study also highlighted commonly held misconceptions of poor physical outcomes caused by alcohol use:

There's always, yeah, there was always, I remember teenage girls magazines when I was young used to always go on about how smoking and drinking affected your hair and skin. I can say hand on heart, since I have had my son and cut right down on my drinking and stopped smoking, my skin has been terrible [laughter]. My skin was better as a drinker, I think I was pickled. I'm not the only woman that's had that experience, so through life experience I've discovered that's a lie. (Cilia, 09/27/12)

Such views reflect the beliefs to which women subscribe, regardless of what they hear or are told by authorities. Personal experience resonates with these women, and is seen to be as truth laden as 'expert' or popular belief.

When asked to consider the impact of alcohol use on women, the issues of female alcoholism and alcohol dependency was only mentioned by one participant, and even then, only 
briefly. When alcoholism was mentioned in response to this question, it was in relation to men's alcoholism and its impact on women in the form of domestic violence.

Probably, alcoholism. Impact on women, I would think, yeah, the sort of violent impact that alcoholism could have on women. ..., yeah, so I think my experience with it and being a woman, is suffering I suppose, you know. (Beth, 09/27/12)

This is not to say that alcoholism and women were never mentioned throughout the entire interview process, as women did express knowledge of female alcoholics or women who drank too much. However, such statements were not discussed in-depth by any of the women when it came to addressing the impact of alcohol on women. Women's alcoholism was not expressed as being bad for women as such, but more a simple fact of a personal existence. It was men's alcoholism that threatened women and had tangible consequences for their health, through domestic violence that came about as the result of men's drinking. I suggest that there are two possible reasons for this. The first is that, as mentioned in Chapter Three, women's alcoholism was considered to be part of the 'hidden' drinking subculture and, therefore, its related impacts were also 'hidden'. Secondly, alcoholism and its impacts are seen as highly gendered in Glasgow. That is, men are the alcoholics that the female participants of this study can easily identify and can talk about as the effects of the men's alcoholic behaviour are visible and understood; domestic violence being the prevailing example identified by participants in this study. Emslie et al.(2012: 482), in their study of men's and women's narratives of drinking in early middle age, also note that men are, "more likely to drink alcohol than women, to drink excessively, and to experience or cause problems related to alcohol". The responses from the female participants of this study would seem to support this statement.

In relation to female health and well-being, women participants saw a direct correlation between men's drinking and its ties to domestic violence in the context of sectarianism and 
football. Many of the women noted the link between excessive drinking and allegiances to the Celtic and Rangers' football clubs, and violence in general in Glasgow. These clubs have a long history of affiliation with religions in Glasgow and elsewhere in Scotland, with Celtic and Rangers supporters traditionally being Catholic and Protestant respectively. There is an ongoing debate over how prevalent sectarian violence still is in Glasgow, however, many of the women stated that it still leads to conflict within communities in the present day (Bruce 2011; NFO Social Research 2003). One participant noted that sectarianism may appear to be on the decline in Glasgow, but such a notion is, in fact, misleading.

Because people aren't honest about it or they've got their own opinions, but they don't want anyone else to know what their own opinions are. So it's [sectarianism] become quite hidden but it's still very much there. You don't have to go far to look at graffiti on walls to see. You don't have to go far to see where somebody's been stabbed and see the football tops that are hung round about the flowers to know that that's been what the problem has been. (Cilia, 09/27/12).

When discussing religious ties to a working class area in Glasgow, Cilia explains how such ties can lead to conflict.

There are a lot of people who come down to use the pubs in [this area] who maybe long ago moved away from the area and their family. But because they've still got a link to the chapel or the Orange Lodge, they still associate with the area so they still come drinking in the area. And that causes unbelievable conflict because it's not in any other areas. They're great, they're fine but it's not an area where you want to be hanging about on a Friday or a Saturday night because of that. (Cilia, 09/27/12)

Cilia explains that people who have left the area still return there to socialize with family and others of the same faith. This association can become problematic when drinking is involved, however, as traditional rivalries may flare up and cause fights between Catholics and Protestants. In this example, the desire to maintain both familial and religious connections through revisiting family and friends of similar background puts people in close physical proximity to rivals. When 
alcohol is added as a means of socializing, it can result in a volatile environment where conflict is more likely to erupt.

Another example of the impact of sectarianism on alcohol and violence was voiced by Lila and agreed with by those present during a group interview:

Just with the tensions between Rangers and Celtic, it's very prominent within Scotland, you know racial kind of tensions, and alcohol just fuels the tensions. [All murmur agreement] (Lila, 08/30/12)

In the context of sectarianism, Glaswegians refer to 'racial' conflicts or tensions between Catholics and Protestants. This is not a simple confusion of terms, but refers to a long and turbulent history between these two religions, and to the 'races' which were believed to make up the majority of the believers. Specifically, Catholicism was linked to Irishness, and Protestantism linked to Britishness (Bradley 2006; 1996). Although 'race' as a biological reality has been rejected, concepts of racial identity persist as socially constructed realities that carry enormous meaning. National affiliations are still understood to be present when labelling people in Glasgow as Catholic or Protestant. Because of these racial tensions, drinking has been banned from football matches and from trains after 9:00 pm in Glasgow. The former occurred because it was felt that alcohol helped exacerbate the rivalries between teams, and the latter because many people would be taking the train home after a match and supporters of different teams would be travelling together. In the following excerpt Joanne explains the connections women in this study make between sectarianism, drinking, and domestic violence in Glasgow.

Yeah, in terms of domestic violence and stuff like that, it's obviously related to alcohol and it's always in the news here whenever there's a Glasgow Rangers' football match. The day after the reports of domestic violence are a lot higher and...there's been lots of research and stuff around so, so, yeah. (Joanne, 08/30/12)

Men's drinking is tied to women's health as the participants believe that it correlates very strongly to the incidence of domestic violence. Sectarianism tensions are used by women in this 
study to explain how football and alcohol influence some men's behaviour which, in turn, impacts women's health and safety in a negative way. To further demonstrate this point Lena, who is originally from Europe but has lived in Glasgow for more than nine years, shares a view of women's interest in football.

My husband thinks (if I can say that) that it is strongly related to the football as well. Football. Because, here, women are most interested about football as well. For me it is totally... again, it is so different. We never watch football at home[country in Eastern Europe], women are not, but here, everybody goes and I think, I'm not saying that they are not enjoying, but it's just a whole drinking session kind of thing. (Lena, 09/12/12)

There is a chain of causality here that is worth stating in detail which ultimately results in the creation of a volatile environment for women. This environment is achieved in the following manner. There is the embodiment of sectarian attitudes (Catholic versus Protestant), in an emotive atmosphere (the football match), which in turn inspires enactment of traditional songs and chants (as is common at most matches) that demonstrate these attitudes and an accepted social norm of drinking copious amounts of alcohol to enhance the overall experience. The outcome of such a combination of factors is clear to the participants of this study. That is, even if women and men start out behaving in a similar fashion at football matches, the final outcome in many cases will be violence, which is directed at the females and perpetuated by the males.

This section has discussed that while women in Glasgow may not overtly link women's alcohol use to their own health problems directly, they are aware that alcohol use does have a part to play in the well-being of women. The links between alcohol use and health outcomes, however, are not necessarily perpetuated by women's alcohol use alone, but also by the way alcohol is used by the men with whom the women have relationships. The participants' tendencies to not dwell on the personal health implications of their own alcohol use reveals that the impact of alcohol on their lives is seen to be predominantly generated by an external, rather 
than an internal, force. Women from this study discussed in-depth how men's drinking can affect the lives of women, but largely ignored the negative effects their own drinking may have. Such concerns over their mental and physical health were mentioned as if in passing and responses did not delve too deeply into women's own risk behaviours or incidences of alcohol dependency. This may be due to the fact that women are more concerned with the social dangers of alcohol consumption, rather than the direct physical harm that can result from drinking excessively. It is difficult to ascertain if this is because it is more acceptable to discuss the actions of men behaving badly than it is those of women, or if the participants in this study feel that women's drinking is tied more strongly to gendered power relations than it is to physical health issues in women.

As drinking is strongly linked with sociality in Glasgow, overindulgence may appear as a less serious occurrence than the actual physical and mental harm resulting from domestic violence. The participants note that, historically at least, the links between alcohol use and physical health are contextualized as applicable to men, and something women 'are catching up to'. Discourse on alcohol use and women's health, may not be common, and may be superseded by the dominant view that alcohol use and negative health outcomes are more of a 'man's problem'. Given the history of women's drinking, as discussed in the previous chapter, women's alcohol use has been on the rise only in recent decades and, as such, more time and more evidence of undesirable health outcomes may be needed before women's health and alcohol use come to be linked in the psyche of the general population.

\section{Section 4.3 Access to Information}

In considering possible reasons for the Glasgow Effect, one theory that has been postulated is that Scottish people do not have the same access to quality health care as other 
populations in the rest of Great Britain (McCartney, G. et al. 2011: 50-51). While this has not yet been proven, it was still important to discuss with the participants if they would know where to look for information on the impacts of alcohol use, should they be interested in acquiring more information. Some participants were able to identify several resources where they could find information on this topic. The most popular resource cited was the internet.

By and large it would be internet access. I think, although a lot of advertisements aimed towards, go to your GP or different health unit. I think most people search online themselves. (Corinne, 09/13/12)

I am a Google queen. Initially I would go to the internet. Probably on the advice of where I looked, a charity or a counselling service. (Beth, 09/27/12)

Other sources included the National Health Service (NHS) Scotland, and other government entities such as the health board, and non-profit charities with an alcohol focus. The participants of one group interview all worked with a non-profit organization that conducted research on social issues including alcohol and, therefore, were very knowledgeable of places to find information on this subject matter.

Roxanne: There is a lot of information from the health board.

Lila: There'd be a lot of statistics for health, alcohol-related whether it's violence, or heart attacks, liver problems and all that. There'll be statistics for it, health-related illnesses...

Deirdre: Alcohol Focus Scotland is based in Glasgow as well, and they do loads of.... I think with the minimum pricing stuff it's quite, there's a lot of stuff going on and out there at the moment. $(08 / 30 / 12)$

While such extensive knowledge may be due to their workplace, other participants were able to identify different places where information on alcohol could be found. These included information from their general practitioner (GP) or some other form of primary health care within the NHS Scotland.

[The] doctor's surgery [office] always has leaflets about alcohol. (Chloe, 09/09/12) 
This was the case even for other participants who worked with drug and alcohol charities, as in the example below where Bea identifies both primary health care and more personal contacts.

I probably, well, everybody has ready access to the internet I think, ya' know I think they could look that up or their GP. Or if they've got any involvement with, even with, a nurse looking after the kids or something. Some kinda medical primary care involvement....Or friends or ask other friends, other, um, folk ya' know from their peer group friends, family. (Bea, 09/22/12)

While Bea, in the above quote, has suggested that people would ask friends and family, the participants of a group interview I conducted expressed a different view, citing concerns regarding issues of personal privacy.

Friends and family maybe? (Group murmurs doubts) There would be issues with confidentiality, I guess. (Megan, 09/09/12)

Statements of doubt amongst this group might be related to the nature of the friendships that are shared among its members. In this instance, the women in the group interview were not longterm friends or particularly close and, therefore, expressed doubt over the parameters of the friendships and levels of trust within that circle. We see that Megan considers that information could be requested from friends or family, but quickly responds to the doubts expressed by the group by citing confidentiality issues. This is in direct opposition to Cilia's opinion, as she felt that, based on her experience growing up in a working class neighbourhood in Glasgow, women would definitely speak to and confide in other women:

Yeah, there is a gossipy culture among women that you would. You'd even do that like, that classic, 'Oh, I know somebody that's got this problem'. Women'll talk about any sort of problems. To be more specific, working class women will talk to anyone about anything. You can have what you think is a completely inappropriate conversation with somebody at a bus stop. I've heard myself doing that before, being like 'Does she know that I'm a social worker? Is this why she's saying that to me?' But, it's not. It's just that people are unbelievably open sometimes. (Cilia, 09/27/12)

This openness and permissibility to speak to family and friends is attributed to the class of the individual and is accepted as a common characteristic that would be held by all women 
with the same socioeconomic status. This demonstrates the shared conceptions that people have within a particular group as described by Bourdieu's habitus, that is "the conditionings associated with a particular class of conditions of existence", made up of, "systems of durable, transposable dispositions, structured structures predisposed to function ... as principles which generate and organize practices and representations that can be objectively adapted to their outcomes without presupposing a conscious aiming at ends" (Bourdieu 1990:53). The habitus, through, "the harmonization of agents' experiences and the continuous reinforcement that each of them receives from the expression, individual or collective, improvised or programmed, of similar identical experiences", results in the conception of a common worldview (Bourdieu: 1977:80-81). In the example discussed above, it is socio-economic status which dictates acceptable behaviour, and it is the enactment of this behaviour and the receipt of an expected response that fortifies the structures of the habitus and the continuation of such behaviour. In this instance, ways of being and behaviour are interpreted as appropriate or not based on where you fall within the class spectrum.

Finally, one participant explained that people would not bother to seek out information about alcohol, as Beth explains:

I would say that the majority for the people I know who drink ..., they wouldn't be. [They would not look for information] They think it's okay. (Beth, 09/27/12)

This further frames the relationship that some participants from this study believe people in Glasgow have with alcohol. High levels of drinking and displays of drunkenness are viewed as commonplace and no cause for concern. If such behaviours are seen as normal, then information to address them is not required and, therefore, need not be sought out. 
While the first part of this chapter argues that women do not readily link alcohol use to women's health issues in a comprehensive way, the current section shows that this does not mean that women are unable to make any links whatsoever between alcohol use and health information. Women in this study showed an active ability to access such information if they so desired. They could easily suggest informational avenues they felt were accessible to them, and their ease in highlighting these indicates the level of alcohol messaging that is prevalent in their city. The Scottish Government and the NHS Scotland are trusted places where women believe reliable information and help can be found. This is in stark contrast to personal relationships which some women felt were less likely to provide support and safety when addressing issues related to alcohol. Interestingly, not all women felt this unease, with one participant even stating that the class in which she grew up influenced the behaviour and expectations of women in such a way that discussing personal issues was seen as acceptable and an appropriate way of engaging with others. Finally, one participant stated that people would not look for information about alcohol use and its impact on health, thereby demonstrating that attitudes towards drinking in Glasgow are flexible enough to absorb occurrences of excessive use of alcohol in such a way that these instances are seen as familiar and, therefore, no cause for alarm.

In some instances, the issues of alcohol use, women's health and access to information overlap. The next section considers two topics where these three factors intersect. These are the issue of accessibility and the understanding of what is considered an acceptable amount of alcohol for women to drink from a health perspective.

\section{Section 4.4 Accessibility of Alcohol and Understanding How Much is too Much}

Women in this study were asked to consider other factors that impact on their use of alcohol and were quick to identify how easy it was to purchase any type of alcohol and ways in 
which purchasing alcohol was rendered more attractive for the consumer. Participants of a group interview were quick to identify accessibility as a contributing factor to increased alcohol use.

Mary: Accessibility.

Megan: You can get it any time.

Mary: Opportunity. It's easy to go to the corner shop to buy a bottle of wine, you know, you couldn't really do that way back in the day.

AJ (Author): So, it's available more places...? (Group murmur agreement)

Megan: And for longer, you know, like 10 o'clock at night.(09/09/12)

Similarly, Lila could identify both places and circumstances that affected the purchase of alcohol in Glasgow.

I think that accessibility to alcohol is an issue. I mean, there's a chip shop and off sales [privately owned shop that sells liquor] in every high street, in every local community so, it's that accessibility and when you can buy alcohol, and drinking until all hours. That never used to happen. You go down to England and you know, closing time [in pubs] is like 12 o'clock. Here it's like, 3 o'clock, 4 o'clock. (Lila, 08/30/12)

Women also noted the increase in the types of venues, beyond the traditional pub or bar, where alcohol could be purchased:

Roxanne: Also, there's drinking in the Cinema now, that's quite a new thing, isn't it? (All murmur agreement). Like, in the West End (All say 'West End' at the same time nodding) there is the [name of popular] Cinema, you go in and there's nice sofas and stuff but when you go in and buy your tickets it's also like, a bar and it sells food and stuff, you can buy a wine and go sit on a nice sofa and watch... You never used to be drinking at the Cinema, did you? (All agree)

Lila: There is also theater ${ }^{7}$ as well. If you go to theater, you can buy drinks at theater. $(08 / 30 / 12)$

When faced with such a plethora of choice of where to drink, most women I spoke with were still uncertain about how much was considered unhealthy to drink. The dominant explanation used to resolve this question in Scotland is a system of 'units'. National Health

\footnotetext{
${ }^{7}$ Theatre here refers to a formal theatre where one may view musicals or plays rather than the movie theatre, which in Scotland is referred to as, 'going to the Cinema'.
} 
Service (NHS) Scotland (2012) informs visitors to its website that "excess alcohol can lead to a number of health problems, and as it is high in calories, it may cause you to put on weight". It also recommends the following method to track your alcohol intake, "men should not regularly drink more than three to four units of alcohol per day," while women "should not regularly drink more than two to three units of alcohol per day" (Ibid). Figure $4.2^{8}$ below provides the NHS Scotland explanation of the volume of a unit of alcohol (Ibid).

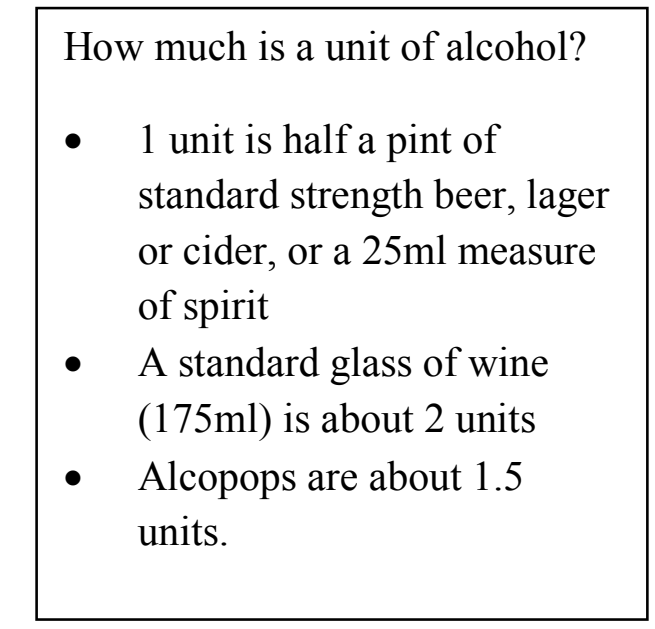

Table 2 NHS Scotland table of 'safe' drinking levels

The participants from this study find the application of units confusing and hard to follow in real world situations for a variety of reasons.

Lila: There's also the issue of how many units of alcohol. And I'm never sure how many units I'm taking so I don't know if there's another way that units can be measured properly or, so advice is given differently for how much is safe to drink, are healthier to drink, rather than by units because I don't think any of us know how much you can, or not supposed to drink in a week or day.

Joanne: Yeah, I think that's true,... I mean I wouldn't be able to tell you off the top of my head how many units and what that would equate to.

Lila: That message is variable. $(08 / 30 / 12)$

Another participant also questions the validity of the units system.

\footnotetext{
${ }^{8}$ NHS Scotland http://www/keepwellscotland.org.uk/giude/alcohol.aspx
} 
Yeah, I still, never to this day, understood why women are supposed to drink less units than men because I'm 5 foot 6 and 13 and half stone. Are you honestly telling me that my body has less capacity than a man who's 5"2 and 10 stone? Cause it doesn't work that way, it works with body mass. So...why is it women have less alcohol units than men do [unclear], what's that all about? I think that is one of the main things that causes women to disregard advice. Is most women do just look at that and go... Why? Why are you telling me that I can drink less than a man? It could be because the way your body processes alcohol. We might have slightly different liver function or something like that. But at the same time, I'm kinda like, 'I thought it was body mass, pretty sure it's down to body mass, so if your body mass is bigger than an average man's, then why should your drinking units be smaller? (Cilia, 09/27/12)

She then continues to explain that there may be an underlying agenda as to why the units system is used:

That's an underlying message that women are supposed to stay more sober than men basically, I think that is the only point in that. Um, it makes me question it and it probably, I can't speak for other people, but it probably makes them question it as well because people aren't stupid. And I think if you do try to tell women that they can't do something the same as a man then that's just a red rag to a bull these days and they will just go (with challenging attitude) 'Aye, right, you think so?' (Cilia, 09/27/12)

In this example, Cilia explains that the units system has an underlying political motive of attempting to control women's alcohol intake. This is achieved by limiting the 'healthy' amount that women are allowed to imbibe in comparison to that of men. Further, this is orchestrated in such a way as to appeal to the women's concern over personal health, so as to avoid the confrontation with women which would result if it was simply suggested that they should drink less than men. Such a statement by the participant indicates a mistrust of governmental authority in this regard, and a fierce pride in the ability of Glaswegian women to think for themselves to the extent that they would need to be tricked to comply with standards that they would normally question.

The units system is also problematic, as the participants feel unfamiliar with present day standard measures for alcohol. 
I think at one time you used to get different size glasses in pubs and different size measures. You know, one unit did used to be one drink, like one gin, you know, everywhere would be one unit. But now with so many people drinking wine, now you get big glasses of wine and wine's stronger than it used to be. So one glass of wine now could be like three units but lots of people probably think that's one. (Roxanne, 08/30/12)

They also remember the former system of measuring alcohol by 'gills' (pronounced jill) which they felt was more familiar:

See, I don't drink wine so for me it's like what used to be a quarter of a gill of spirit that you would have and now it's a fifth of a gill. So I don't know what that is in unit terms so I could have, I could be well over my limit and unit intake but I do not know. (Lila, $08 / 30 / 12$ )

There's a measurement for it ... It's spelt G-I-L-L...That was the traditional measure for spirits. Now, it's in millilitres because you've got like quarter gills and half gills. 'Cause there's actually a pub in [neighbourhood in Glasgow] called that 'The Quarter Gill' which obviously meant that they gave a bigger measure because they gave quarters and not eighths. (Cilia, 09/27/12)

The above excerpts tell us that women in this study are aware that there are healthy limits for alcohol consumption but are choosing not to adhere to them, citing confusion as the reason for non-compliance. This could be due to the fact that they do not want their alcohol intake limited by others or because, as we have seen so far in this chapter, they do not equate female health problems with female alcohol consumption directly and therefore see no need to limit their intake. What the participants also reveal is a contradiction between the spheres of health and industry in Scotland, as each pursues its own particular mandate. On the one hand, the Scottish Government is providing increasing number of liquor licences to new and increasing numbers of venues to render alcohol universally accessible, thereby creating new jobs and responding to consumer demand while, on the other, the same government publicizes information about 'safe drinking' levels, which the general public find confusing and misleading. This highlights the dual agendas that co-exist within the Scottish Government as it attempts to navigate the diverse needs of both the public health of its populace, and the creation of new financial enterprises 
within the country. This conflicted duality within the Scottish Government is further exacerbated by Scotland's relationship with stakeholders within its own alcohol industry, the United Kingdom and the European Union (EU). It is to a discussion of these stakeholders that I now turn.

\section{Section 4.5 Political Economy \& Scotland - What's Alcohol Got to do with it?}

A recent article in The Herald newspaper (Bell 2012: n.p.) states that, "alcohol-related harm could cost more than $£ 1,000.00$ per person" in the population included within the remit of Glasgow's City Council. This per person cost estimate includes, "the financial impact on the health service of the city, the cost of crime, providing social care and also the loss of productive capacity," thereby indicating the links between various sectors of society in Scotland that the Government considers to be affected by alcohol use (Ibid). Elvins (2009: 53-56) notes that the Scottish government responded to public concerns over excessive drinking by enacting special legislation that was intended to act as an impetus for social change within Scottish society. This legislation, although similar to that passed in England and Wales, contained one additional licensing objective: that of "protecting and improving public health" (Ibid). This inclusion, Elvins (Ibid) states, "suggests ... that the Scottish legislature has embraced a more holistic view of how different aspects of the potentially negative consequences of alcohol use are interrelated".

The work of the Nicholson Committee, created in 2001, was to review, "liquor licensing and practice in Scotland, with particular reference to the implications for health and public order" (Ibid). A relevant outcome of the committee's work was to declare that changes to licensing laws themselves could not bring about societal changes to the excessive use of alcohol and its consequences, as "many of the problems associated with [these] are deeply engrained in the Scottish psyche, and reform of the law will not itself bring about changes" (Ibid). The 
Scottish Government, therefore, is providing a contradictory public message. In this instance, the Scottish Government takes action to address alcohol use, framing it as an activity that can have negative impacts on public health and Scottish society in general, and then undermines its actions by assigning blame to its citizenry. It offers public health advice, incorporates legislation that is meant to address public health and then declares that, despite the actions the government is taking to address alcohol use in Scotland, it is, in fact, powerless to intervene as the real responsible party is the Scottish people and their 'psyche'. An interesting choice of words as a person's psyche is, by its very nature, an inaccessible entity far beyond the governments' remit (or anyone else's, bar a psychotherapist, for that matter). This assignment of blame to the people, thereby releases the government from responsibility in addressing the problem.

The media is quick to note hypocrisy in tangible examples such as the one shown below from the Metro Newspaper (Gairns 2012), in which a concerned citizen notes that the Scottish Government claims to be concerned with controlling public drinking, and, yet still manages to establish its own bar in the Parliament building. Such an example, however, also serves to demonstrate the degree to which social drinking is normalized within Scottish culture.

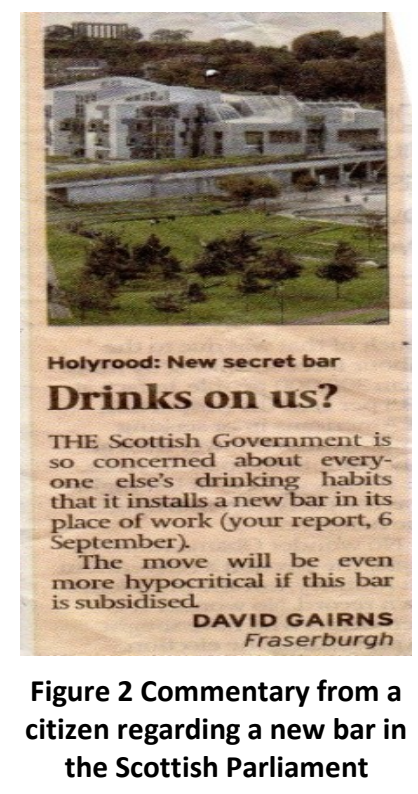


Connectedly, participants commented on how new opportunities to buy alcohol were becoming commonplace in Glasgow, and increasingly geared to make buying alcohol more appealing to the consumer.

Megan: Well, Marks and Spencer's have their meal for a tenner [£10] and that includes a bottle of wine. (Group all agree enthusiastically)

Chloe: That's such a selling point isn't it, for a lot of people. And it's not the meal, is it? (Laughter)

Mary: Just makes it more acceptable to have a bottle of wine to choose tonight. $(09 / 09 / 12)$

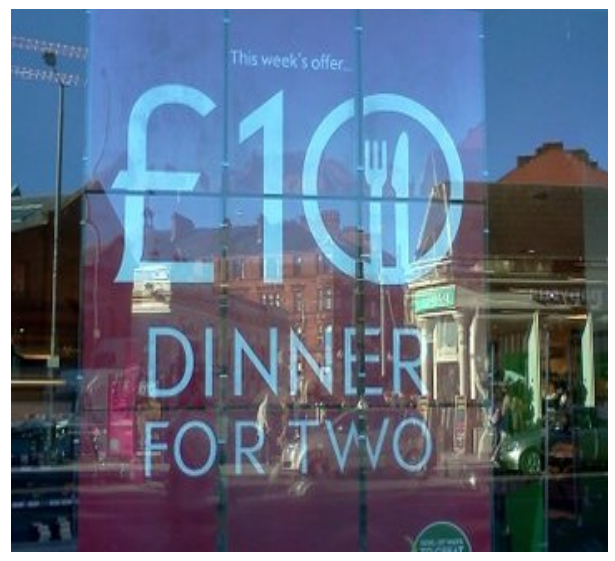

Figure 3 A sign in an upmarket grocery store advertising a dinner special that includes a bottle of wine in the price

While there are outstanding changes to be made to the Licensing (Scotland) Act 2005, the legislation itself has the following 'licensing objectives': preventing crime and disorder; securing public safety; preventing public nuisance; protecting and improving public health; and protecting children from harm (Licensing (Scotland) Act 2005: n.p.). These are aligned with the Scottish government's statement in its document, "Changing Scotland's Relationship with Alcohol: A Framework for Action" (2009), that identifies the need for "sustained action in four areas: reduced alcohol consumption; supporting families and communities; positive public attitudes, positive choices; and improved treatment and support" (Ibid: 5). However, another 
statement from this document serves to highlight the difficulties that are evident in trying to address the needs and wants of retailers and consumers, while still addressing the government's desire to limit damage caused by alcohol use in the country.

We note the argument that retailers should be able to promote and price the goods they sell in a way that attracts customers from their competitors. The amount of advertising by supermarkets for cheap alcohol over recent months suggests that alcohol does play a key role in determining where people shop. However, we return to the point that alcohol is not an ordinary commodity. While it is understandable that retailers wish to keep prices low to retain customers and attract new ones, we consider it undesirable for alcohol to be discounted in a way that can lead to increased consumption and harm. The long term costs of excessive alcohol consumption far outweigh the short term benefits and we believe this is an area where competition is failing the consumer (Ibid: 11).

The document further goes on to state that the government's approach is "targeted at everyone, including the 'ordinary people' who may never get drunk but are nevertheless harming themselves by regularly drinking more than the recommended guidelines" (Ibid: 7). This statement serves to demonstrate that alcohol is not being demonized by the government, but framed as a commodity that needs to be controlled to be properly enjoyed. I would argue that such a stance is necessary, as it serves to address the whole population. A standpoint that only targets 'problem drinkers,' or those that commit offences when drunk, would allow those who embrace social drinking as an enjoyable pastime to ignore the efforts that their government is making and to render any recommendations as inapplicable to their own lives.

Tellingly, the Scottish Government has not only its own internal workings to consider. It also has to contend with competition from the neighbouring alcohol industry in England. Some participants noted that the competition between Scotland and England to attract customers to purchase from their respective industries was on-going.

Iris: But have you seen the reports in the paper saying that some English authorities are trying to entice Scots to go down there to drink more because it's going to be cheaper? 
Chloe: And to buy their alcohol, stock up the cars and drive back.

Mary: Tesco's in Carlisle [a nearby English town just over the border] then! [Laughter and agreement] $(09 / 09 / 12)$

Media coverage of government actions against alcohol is pervasive in Glasgow. And, as is usually the case with western media, the reports tend to concentrate on the negative and sensational rather than the positive. An example of this can be found in the news clipping presented below, which states that while drinking rates have fallen in Scotland, there is no cause for celebration as Scots are still buying more alcohol than their neighbours in England and Wales.

\section{Fall in drinking levels no reason for cheers} sCOTS are drinking less alcohol - but still buying 20 per four per cent between 2010 and 2011 . However, alcohol cent more than their English and Welsh counterparts. sales remained ten per cent higher than in 1994 and 20 An NHS report analysing retail sales revealed yesterday per cent more alcohol is sold per adult in Scotland than that the amount of alcohol sold north of the border fell in England and Wales.

Figure 4 An article regarding high drinking levels in Scotland

Such stories provide fuel to the Scottish Government's attempts to incorporate minimum pricing towards units of alcohol. This refers to the enactment of legislation that will dictate that the price of a unit of alcohol sold in Scotland cannot be less than 50 pence (BBC News 2013). Minimum pricing was often in the news during my time in Glasgow and many participants mentioned it during the interviews.

Well, up until the whole minimum pricing thing, alcohol is much cheaper now than it was, say 10 years ago compared to like people's income, how much money they had to spare. (Roxanne, 08/30/12)

During one group interview, while discussing the effects of the recession on alcohol use, participants queried whether changes in legislation on minimum pricing may lead to societal changes. 
Chloe: I don't know if the recession has affected drinking, really, other things...

Mary: It's affected people going to the pubs and alcohol sales have shot up.

Chloe: Yeah, cause so many pubs have shut, haven't they? (Murmured agreement)

Iris: Yeah, to do with the smoking ban. A lot of pubs have shut.

Mary: ... People just couldn't afford to go out, and it was cheaper for people to buy in bulk, or, like, say have friends round.

Chloe: When you think, like, a glass of wine in the pub is, like, $£ 4.50$ in the town, or something isn't it, while you're getting the bottle for 5 or 6 pounds.

Iris: I suppose that might change in the next wee while when they bring in the minimum pricing.

Megan: It's not going to affect the 5-6 quid [£] bottle of wine though is it?

Chloe: No, it'1l be the cheaper 2-3 pound bottles of wine. $(09 / 09 / 12)$

Minimum pricing is of great interest to the Scottish Government, who believe it to be, "the most effective and efficient way of reducing alcohol consumption and hence alcohol-related harm, and believes decisive action to address the affordability of alcohol is required" (NHS Scotland 2013). It is promoted by the Scottish Government as part of their larger framework for, "reducing the health impacts of hazardous alcohol consumption" (Ibid). The reasons for this are self-evident, as reducing health impacts also means reducing costs to the public health system which is funded by the Scottish Government. Health promotion and preventative measures are useful to save money and avoid ill-health of the population. Alcohol Focus-Scotland, a nonprofit organization whose aim is to "reduce the harm caused by alcohol", is also in favour of minimum pricing as they maintain "there is a clear link between consumption and price - as the price of alcohol has fallen, consumption and related harm has risen. Alcohol is $44 \%$ more affordable today than 30 years ago" (Alcohol Focus 2013: n.p.).

Not all parties, however, are supportive of minimum pricing. The European Union is fighting the Scottish Government and, the "European Commission lodged a formal objection to 
the policy" (Gardham 2012: 2). This objection is presented in terms of Scotland breaching the terms of free trade across Europe, thereby impacting on countries where alcohol can be manufactured more cheaply before being exported to Scotland (Ibid). Internally, the Scottish Whiskey Association (SWA) has recently lost an action against the Scottish Government, hoping to appeal the decision taken by Members of Scottish Parliament (MSPs) in May 2012 to pass the Alcohol (Minimum Pricing) (Scotland) Act 2012 (BBC News 2013). It is believed that the actions of the SWA are due to the fear that minimum pricing will affect their profits (Ibid). As similar legislation has not yet been passed in England and Wales, Scotland is finding itself at the forefront of a debate that may have unforeseen future consequences (Ibid). As nationalism grows in Scotland, there grows with it uneasiness as to whether or not Scotland, should it become independent, will be welcomed into the European Union (EU) as a country in its own right (The Evening Times 2012a \& 2012b). Such an outcome is far from certain and it appears the Scottish Government is currently working in the best interests of its citizenry and its own bottom line, at the risk of possibly alienating future relationships with the EU.

As discussed in Chapter Two, a critical medical anthropological perspective requires that we consider the political economy in which behaviours of a cultural group are carried out. This section has shown that the drinking practices and opportunities to drink in Glasgow are not only related to historical practices, but also to the larger institutions which control how, when and where alcohol consumption may take place. The priorities and subsequent actions of the Scottish government, and its relationship with the EU, are key factors in understanding how Glaswegians' relationship with alcohol is formed and the drinking opportunities which are available to them. 


\section{Section 4.6 Summary \& Conclusion}

This chapter has presented findings from this study in relation to the impacts of alcohol use on women. The women interviewed discussed how alcohol use impacts their health, and that while information on alcohol use is accessible, it is often confusing and hard to incorporate in a practical way. Women appear to be less concerned with how alcohol affects their physical health and, are more attuned to the negative impacts it could have on their personal safety and emotional health. Interestingly, many participants wanted to talk about the impact of men's alcohol use on women's health and voiced a general acceptance that there was a strong link between men's alcohol use and the incidence of domestic violence. This chapter further contextualized alcohol use in Scotland, and provided insight into the competing factors that the Scottish Government must consider when it undertakes any action to address alcohol use and public health in the country. Relationships with a variety of stakeholders, both internal and external to the country, mean that the Scottish Government faces challenges when addressing the needs of its population, which result in initiatives that are contradictory and may simply cancel each other out so that action becomes, in effect, inaction. The next, and final, chapter of this thesis will present final issues for consideration, summarize overall findings from the project, and provide recommendations for further research. 


\section{Chapter Five: Summary and Conclusions}

\section{Section 5.1 Introduction}

This thesis shows that women's alcohol consumption is strongly tied to other aspects of their lives such as gender, identity and issues of social class. It reveals that most women in this study do not readily connect their own drinking habits to their overall physical health, but focus instead on men's drinking practices and their ties to larger sectarian forces within communities in Glasgow. When women do mention health issues related to alcohol consumption they are usually in relation to maternal health issues, such as the perils of drinking during pregnancy, or to how women are 'catching up' to men in alcohol-related health problems. Finally, this thesis discusses the relationships between various competing stakeholders in Scotland and the European Union who have a vested interest in the alcoholic drinks industry and related health policy, and that the Scottish Government has a complicated task to navigate the various interests of competing factions.

It is now necessary to explore female drinking in view of what has thus far been presented. To this end, I will consider women's drinking in Glasgow from the individual, the societal, and the institutional viewpoints. This will serve to both summarize the findings presented herein, and to present final thoughts on women's alcohol use and links to the Glasgow Effect. This triad has previously been introduced in Chapter Two, where it was used to explore relations of power relating to the content found within this thesis. Just as drinking practices are dictated by culture so too is, "knowledge relating to the body, health and illness...culturally constructed, negotiated, and renegotiated in a dynamic process through time and space" (Scheper-Hughes and Lock 1996: 43). Consideration of these three levels of analysis will capture not only the lived reality of Glaswegians and societal factors relating to female alcohol 
consumption, but will also contextualize women's alcohol use within a political economy where influences from larger institutions in society can be considered. Finally, this chapter will provide recommendations for further research in the area of anthropology and alcohol studies.

\section{Section 5.2 Three Levels of Analysis and Women's Alcohol Consumption}

Throughout this thesis women's alcohol consumption in Glasgow has been presented as a complex phenomenon that: conveys meaning of gender, identity and class; requires consideration of health and wellbeing that are linked to larger societal factors; and, exists within a larger political economy which exercises control over accessibility and consumption of alcohol. Final consideration of female drinking in Glasgow, therefore, requires that the topic be considered from three distinct, but interrelated, levels of analysis. As stated earlier, these are the individual, the societal and the institutional. Each of these will now be examined in turn.

The first level, that of the individual, takes into consideration the lived experience of a person. The women in this study related experiences in which alcohol consumption had a direct impact on a variety of personal elements. The first experience, discussed at the beginning of Chapter One by several of the women, presented the impact that alcohol has on the emotional health of a person, particularly on those occasions when women drinking alcohol act in ways that later cause them to feel guilt or remorse for their actions. The examples given were related to two specific experiences: sexual behaviour that, in retrospect, was regretted, and, conflicts that arose between friends due to excessive drinking. Such examples show how an individual woman's sense of self, the use of her body, and her relationships with others could be negatively impacted by alcohol use in a deeply personal way. In fact, when the issue of regrettable past events and guilt was mentioned during one group interview, many of the women visibly shuddered, illustrating the physical manifestation of unease that such memories and related negative feelings 
produce in the individual. This 'shuddering' is the physical embodiment of the emotional impact that adverse alcohol-related events had on the participants.

Another example was the discussion regarding lifestyle and the links participants drew between images from the TV program, 'Sex and the City', and drinking practices. Women spoke of dressing up, doing their makeup, and donning high heels before going out as a means of embodying the sophisticated characters they found so appealing on the television show. This behaviour served to make them 'feel' as though they were partying in New York City, even though they were actually going out in Glasgow.

Similarly, many women articulated that being able to drink what they wanted, when they wanted, and where they wanted was empowering as it was a public statement of their equality to men. They could physically behave as men, ergo, they were equal to men. This sentiment, however, was juxtaposed against a feeling of personal vulnerability that many women noted in viewing other women who had imbibed too much alcohol. A drunk woman was in an altered personal state, and was viewed by the women of this study as physically vulnerable, her body at risk of harm. This duality of feeling, empowerment versus fear, discussed by the women in this study when seeing a woman who appears to be intoxicated in public, demonstrates dominant discourses of women in Glasgow that are experienced on the personal level. On the one hand, we have women enacting powerful male traditions (i.e. drinking in pubs, going out with friends to socialize with drinking, celebrating with alcohol, etc.), and, on the other, we have women who identify with the drunken women they see, noting the vulnerability of women who are not 'in control,' and fearing for their safety. Such views underpin the juxtaposition of female roles in western society; a woman can be empowered in her actions and demeanour, but underpinning 
this stance is the belief that women are inherently vulnerable and need to conform to gendered expectations, both physically and mentally, to truly be safe.

Finally, when discussing the impact of alcohol on health, the women in this study were less concerned about physical effects on women's health in general, and much more likely to address how drinking impacted them individually. That is, how bad the hangover would feel if one drank too much and what other areas of life would be affected by excessive alcohol consumption, for instance, not being able to enjoy time with family due to a hangover. Similarly, as discussed in relation to Paechter's 'communities of practice' in Chapter Two, women were expected to be 'self-policing' when drinking. This attention to individual selfregulation is required if one wants to remain part of a group, as challenging the boundaries of a group can result in ostracization. Chapter Three discusses how gender expectations require that women maintain a high degree of self-awareness when drinking if they hope to avoid incurring negative judgements regarding their morality or their inability to look after themselves.

The examples discussed above show how the individual is affected by alcohol use and the meaning that is attached to female alcohol consumption on the individual level. But what of the influences of society at large? This leads us to consideration of the second level of analysis which refers to culture, nature and society, and the enactment of cultural scripts that are acceptable within a particular time and place.

Women noted that their drinking practices were tied closely to the varying roles that they enacted during the lifecourse. For example, pregnancy was a physical state that for most women required abstinence from alcohol. Having children required a personal shift in behaviours and expectations of how leisure time would be spent in light of new priorities and responsibilities. Such a view also reveals the importance placed on the nuclear family within this society and, as 
discussed in Chapters Two and Three, the gendered expectations placed on women to conform to specific ideals of motherhood that are common in the West. According to the women interviewed in this study, a woman's behaviour is expected to change when pregnant or once she has children, as they become the priority and the focal point of daily living.

Perhaps the most illustrative example of this societal level is the extent to which class is assumed to dictate drinking practices in Glasgow. Class identity and values are reflected in people's drinking behaviours, according to the mature women participants in this study. Interestingly though, many of the participants in this study simultaneously accepted and rejected class distinctions, on the one hand explaining that they were not snobs and, in the next breath, stating that those from the lower social classes exhibited the most anti-social or unhealthy drinking practices. The upper classes were also identified as being excessive drinkers; they simply could afford more expensive types of alcohol. This shows an internalization of a societal conflict within some participants as they attempt to reconcile the competing societal norms of a hierarchical class system and a larger, Scottish national identity which rejects a class system, in contrast to the English 'other' who accept this institution. The pathology of excessive drinking, however, was the same for both classes.

These specific distinctions between class behaviours and the bodily consumption and impact of alcohol mirror class relations within this context. More specifically, participants are commenting on their own place in the class system in Glasgow, by stating that they do not behave as badly as the lower classes, and, yet, they are also not as indulgent as the upper classes. One sees in such comments a moral value placed on being middle class, framing that level of class distinction as the reasonably behaving class that balances the excesses of the others. Even though many of the women I interviewed were well educated, they still discussed themselves as 
neither lower nor upper class, thereby identifying themselves as middle class by default. This was also exemplified by comments from Cilia when she discussed the relevance of 'being hard' in Chapter Three. Such statements exemplify the social value that is attached to the middle class in Glasgow, and reflects the pride felt in coming from a hard working, middle class background that is evident in the numerous working class neighbourhoods that make up the city proper.

Considering the participants' discussion regarding health concerns and alcohol consumption, we also see evidence of its relation to the 'ills' of society. An example of this can be found in Chapter Four where women were less inclined to speak of their own drinking behaviour and its impact on their health. Interestingly, what was of paramount importance to them was how men's drinking behaviour was linked to women's well-being. The women in this study established links between men's drinking and women's suffering, drawing particular attention to domestic violence. This connection was presented by the participants through their discussion in Chapter Four of the ties between male alcohol consumption, football (i.e. soccer), and sectarianism, and how these intersect to create an unsafe environment for women. Identifying domestic violence as a consequence of men's alcohol consumption denotes the 'sickness' prevalent in society that allows for women to be facing this threat. Women's comments in this context reflect the unequal and gendered power relations between men and women, articulated through examples of football and its ties to sectarianism.

Women did discuss their personal health and its relationship to their own alcohol use, although in much less detail. When they did discuss these topics, they often contextualized them in two distinct ways. The first context was to compare women's alcohol-related ill health to that experienced by men. That is, women in this study stated that women in Glasgow in general were said to be 'catching up' to the men in regards to physical health problems related to alcohol (e.g. 
cirrhosis, liver failure etc.). As stated in Chapter Four, it may be that the harm incurred by alcohol use has historically been seen as a 'man's problem' and, as women drinking socially is a newer phenomenon, a connection between women's alcohol use and women's ill health has yet to be established in the psyche of the general population. The second context relates to maternal health issues. This was exemplified by comments that there were still too many pregnant women drinking heavily, and that if a woman chose not to drink, the first reason that would spring to mind for this abstinence was that the woman must be pregnant. This tendency to ignore other possible connections between alcohol use and women's health has been found in other studies, and illustrates how women's health issues are often conceptualized in relation to their traditional role of child bearers and mothers. Other health issues unrelated to motherhood (e.g. cirrhosis, liver failure, etc.) are overshadowed by this historical and dominant view of women, even though such issues may be equally important to a woman's overall health and wellbeing.

Glasgow, as already noted by comments from the participants, is often viewed by nonScottish people as a hard-drinking, dangerous city. All of the participants in this study agreed that this was a commonly held belief, but some also stated that the city was probably no worse than any other big city, or, that the drinkers that caused trouble in Glasgow were actually from areas outside of the city and only came to the city centre to drink and party. Despite the negative connotations that may be ascribed to this view of Glasgow, some participants made comments regarding how many Glaswegians try to live up to the 'hard-drinking', tough reputation, and the women in this study were quick to discuss how people may engage in a sort of one-upmanship when discussing drunken behaviour and related problems; a sort of attitude that would convey, "You think that's bad! Well, listen to this!" The perpetuation by Glaswegians of this 'hard drinking' reputation, I believe, serves to fulfill two functions. The first is to serve as an exclusionary mechanism for those not from Glasgow, who do not share the same history and 
background. The second reinforces the first in that it serves as a means to embrace the sense of 'other' that is often bestowed on the Northern populations of the United Kingdom. Put simply, Glaswegians support such stereotypes as they glorify characteristics that are valued in their population, such as the ability to endure hard times and still enjoy life, while at the same time, accepting this reputation as proof that they are not like their neighbours to the south in temperament. Glaswegians are proud to be tough, and proud to be Scottish, as this sets them apart from the rest of Great Britain and fuels pride in a communal 'National Self'.

This 'National Self' can be further expanded to illustrate the institutional level of analysis, which refers to how the state or larger institutions can exercise control over its citizenry. Foucault (1990:140) referred to this as 'biopower', seeing it as the "explosion of numerous and diverse techniques for achieving the subjugation of bodies and the control of populations". This is evident in the attention and effort the Scottish Government is applying to policy related to alcohol use in Glasgow and elsewhere in Scotland. Identifying alcohol use as a priority, and linking it to concerns about public health, demonstrates the ways in which governments can engineer their policies to appeal to a population and respond to its health and social needs. The way in which larger institutions prioritize issues, such as alcohol consumption, and the actions that they take to address these issues, are based on particular values that these institutions deem to be important. Such beliefs filter down to the populace through policy creation and media institutions, thus informing the public of the 'acceptable' way to use alcohol. As discussed in Chapter Four, such beliefs are internalized to such an extent that people begin to act in particular ways without being coerced.

A prime example of this was discussed in Chapter Four, when participants expressed dismay at being unable to understand the 'system of units of alcohol' that the Scottish 
Government recommended to determine acceptable drinking levels for men and women. The confusion in adhering to this system was mentioned by several participants. They sought to behave in the 'acceptable way' as outlined by the units they were 'supposed' to imbibe according to the Scottish government, and expressed distress when they were unable to do so. In short, their own propensity to judge personal safe drinking levels had been superseded by their desire to conform to the guidelines set out by larger institutions.

While the Scottish Government does articulate alcohol use and public health as a primary concern, it also ties alcohol consumption equally to a variety of other social and financial concerns. In the Scottish Government's report (2009: 6), entitled “Changing Scotland's Relationship with Alcohol: A Framework for Action”, it states:

There are social and economic costs of excessive alcohol consumption. Excessive drinking can cause families to break down; it can result in crime and disorder, especially at week-ends; and it causes loss of productivity through sickness. We estimate that alcohol misuse costs Scotland $£ 2.25$ billion every year.

Alcohol is also noted to be having devastating effects on the health of the population with some of the "fastest growing rates of liver disease and cirrhosis in the world" (Ibid). Connections are made here between alcohol and other facets of society, such as health, families, crime and economic losses for the country. Such a view illustrates the Scottish Government's acceptance of the pervasive nature of the impact of excessive alcohol consumption, and its ability to infiltrate social spheres that, at first glance, may appear unrelated. Hence, changing negative, anti-social, alcohol-related behaviour is a priority area for the Scottish Government, which is seeking diverse ways to address such issues.

One such avenue for change was discussed by the participants of this study, and addressed in Chapter Four: minimum pricing of alcohol. The enactment of minimum pricing 
legislation dictates that units of alcohol must be priced at 50p/unit. It is an example of a tool used by the state to bring about a specific behaviour from its populace, the instruments of state that Foucault refers to as "institutions of power" (1990: 141). In contrast to biopower, other efforts at containing the negative effects of excessive alcohol consumption on the citizenry have included a larger police presence in the city centre at night, as well as, the employment of taxi marshals in the city centre (Elvins 2009: 55-58). Such measures have been employed not only to protect the people, but also to cut down on costs related to violence and property damage within the city related to alcohol misuse.

The institutional viewpoint also allows us to consider dominant discourses apparent in the comments from women in this study and what they tell us about the power relations in which these women find themselves. Female alcohol consumption has changed greatly in recent decades, but many women still remember a time when women who drank in public were condemned as 'women of ill repute' or labelled as alcoholics. Such women were seen as great threats to the hegemonic structure that placed the rightful place of men in the public sphere and the women in the private (domestic) sphere. Similarly, Jackson and Tinkler (2007: 261-264), in their comparative essay on modern women of the 1920s and 'ladettes' of the early twenty-first century, note that perhaps the biggest threat that these groups presented was to "disrupt the gender order". As we have discussed, gender roles are changing, but women are still conscious of a profound vulnerability that appears to cast a shadow over the rights and freedoms they feel they are embodying when drinking alcohol. We have also seen that women's behaviour tends to change markedly when they choose to have children and, I would argue, such changes are not expected by society for men to the same degree in similar circumstances (i.e. when a man becomes a father). This would support my argument that while women's freedoms may have changed in recent decades, their choice of actions is still formed and bound by gender 
expectations that are socially constructed. This leads me to question whether the links between sectarianism, alcohol consumption, and health concerns that mature participants in this study identify are articulated as a passive form of resistance. That is, by drawing attention to the threat of domestic violence, which is related to alcohol consumption and football, women are speaking out against such threats publicly, indicating the unacceptability of such behaviours.

Whether, in fact, women are at increased risk of health problems related to alcohol use or not, what is telling are their responses to health questions. Many of the participants chose to frame women's health problems caused by alcohol consumption as perpetuated by male drinkers. Few of the participants noted physical problems directly, which leads me to question if their right to alcohol consumption has been hard won and, as a result, perhaps they are less apt to criticize this relatively new found freedom. Their relative silence on alcohol-related health problems of women may, in fact, be a commentary on the still uneven gendered power relations they encounter in their daily lives. What we are seeing in the comments of the women who participated in this study are the connections between drinking alcohol and other facets of their lives, what meaning they take from these connections, and which are most important to them. Our next task is to assess the connections between women's health, alcohol use, and the Glasgow Effect, as articulated by the women in this study.

\section{Section 5.3 Women's Alcohol Use and The Glasgow Effect}

It has already been stated elsewhere in this thesis that causes of the 'Glasgow Effect', that is, the high rates of poor health in Scotland and in Glasgow in particular, are unknown. Many theories abound and this thesis is not claiming it will be able to single out one cause over another. This is due, in part, to the short time the author spent in the field and the limited number of participants that could be interviewed. It is also due to the fact that any large health 
phenomenon needs to be assessed in-depth, and to consider a multitude of factors (i.e. economics, history, current health policy, health practices, among others). Such an analysis is beyond the remit of this thesis. What is possible is to review the discussion of women's health and alcohol use by the participants of this study, and assess what, if anything, this information can tell us about the 'Glasgow Effect'. This exploration will, in turn, pave the way forward for us in terms of considerations for future research. To begin this process, National Health Service (NHS) Scotland will now consider in more depth the way in which the mature women in this study responded to questions regarding women's alcohol consumption and health.

The participants in this study do not choose to speak at length about how their own alcohol use can impact their health. When such a connection is stated, it is in passing, with little detail given, or in comparison to the poor health of men, which is attributed to alcohol use. There may be several reasons for this. The first is that women are slow to criticize the negative outcomes that drinking alcohol may be causing them, as they are still in the 'honeymoon' phase where they are enjoying this new freedom. As such, the tendency of women in this study to largely ignore direct links of alcohol consumption to negative health outcomes may be an act of resistance. Rolfe et al. (2009: 331) note this resistance in their discourse analysis of the accounts of women heavy drinkers. In their study they see that women "directly identify what they see as common stigmatized subject positions of women drinkers, and in identifying them, resist them" (Ibid). That is, the women will discuss perceptions of women drinking publicly and then speak about or behave in a manner which opposes these perceptions. The women in that study simply resist negative judgements of female drinking, such as being labelled 'amoral' or 'unfeminine', by acting as if they do not exist or by heeding them as little as possible. The women in this thesis may also be reacting in this way, in paying scant attention to health difficulties that may arise from drinking. 
The second reason that women may not be addressing issues of women's health and alcohol consumption, may be that there has not yet been sufficient time to see the effects that increased alcohol consumption has had on the majority of women living in Glasgow. Historically, men have been the segment of the population associated with alcohol, both its negative and positive outcomes (Emslie et al. 2012; Plant 2008). As such, more time and more research on women and the negative effects of alcohol consumption are needed before current beliefs are challenged and, consequently, changed. Furthermore, if, as we have already discussed, women are still associated with 'traditional' gender roles related to motherhood, then women's health research and its associated funding may be concentrated in those areas rather than alcohol-related health research. Finally, Glaswegians might not yet be able to link health effects to female alcohol consumption in a meaningful way. The women in this study single out other areas of health that have a bigger impact on the female population as a whole (for example domestic violence) that loom larger in the female psyche, and that appear much more threatening than poor health outcomes, the roots of which they do not yet recognize.

Another factor that impedes our ability to link female alcohol consumption to the Glasgow Effect is the lack of evidence that is accessible in some aspects of women's drinking behaviours. For example, many women in this study noted the existence of 'hidden drinking' among other women. By the very nature of being hidden, it is impossible to assess what impact, if any, such drinking may have on women's health, or how pervasive it is in current society. Similarly, many participants noted that older women are now drinking more. The effects of an older woman's drinking may not yet be apparent or as well recognized as those which result from a lifetime's drinking. As well, it is safe to deduce that older women's drinking is not as visible as young women's or men's drinking and, therefore, attracts less attention when it comes to research funding for projects looking to further explore this group. 
Finally, the Scottish Government has only recently won its battle to legislate minimumpricing of alcohol and, as such, we do not yet know the impact this will have on female drinking, be it old, young, or hidden. In a cityscape that is seeing a variety of changes both in female drinking practices and female rights and freedoms, it may be difficult to capture the changes that are taking place and to reveal connections to health outcomes. We have also seen in this study some evidence of a public mistrust of the Scottish Government, related to the information that they provide to the public (e.g., units of alcohol for men and women). This could have the result that even if women did know of reports that link their alcohol consumption to negative health outcomes, they may not believe them or they may dismiss them out of hand in favour of their own personal experience.

While definitive links relating women's drinking to the poor health outcomes in Glasgow have not been revealed within this study, this research has provided a snapshot of what the women in this study deem important when considering female drinking practices and how such practices are judged by those living in Glasgow. It has also revealed how the three levels personal, societal, and institutional - are embedded in the political economy of Scotland, which has an impact on health outcomes related to alcohol use. Further exploration of such concepts and themes could provide further evidence of connections between women's drinking and negative health outcomes. As such, in the final section of this chapter I turn to considerations of areas of further study.

\section{Section 5.4 Future Areas of Research}

One area of study that could provide us with more insight is that of 'hidden' drinking and older women`s drinking practices. Holloway et al. (2009: 827) note that, "rural and older women are less likely to be pub drinkers". In the example given from their study, this is in part due to 
the fact that the woman interviewed was a widow and her life became increasingly "privatised" (Ibid). Thus, I contend that 'hidden drinking' need not be purposely hidden (although this could be the case), but hidden in the sense that it is removed from public view and, therefore, harder to assess. Studying the drinking practices of older women might therefore provide important information regarding current behaviours and attitudes towards 'hidden' drinking, and identify social and cultural factors that dictate that it becomes hidden. Another area that could be further explored is that of drinking and entertaining at home. Again, this practice is removed from public view but, interestingly, it is also linked to social class. As noted earlier in this thesis, people who choose to entertain at home tend to be those who have the money to afford to do so, the middle to upper classes. As such, once again, their drinking is exempt from public view. Future research must take into consideration socio-economic differences that can dictate the location and type of drinking in which people engage and how these differences may also affect personal views on health.

A useful comparative study could be achieved through a more in-depth look at rural and urban conceptions of women's drinking, and how the related social and cultural landscapes impact on these. This could be linked to differences between acceptable patterns of censuring women drinkers and how public and private spaces are connected to gender, meaning and performance. Regional variances and historical uses of alcohol could be investigated to identify if female drinking in rural and urban environments has changed in similar ways within a similar timeframe.

Finally, as we have already seen in this thesis in relation to sectarianism, religion can have a powerful effect on drinking patterns and related behaviour. A further area of research could explore if there are other ways in which religion has an impact on the alcohol use of 
women and men in Glasgow. This could be an area of study which could include the growing numbers of new immigrants belonging to diverse faiths coming to reside in Glasgow, and could examine how they are able to incorporate themselves into an environment where alcohol consumption is valued by the majority of the population. Such a study would also necessarily consider the relationships burgeoning between native Glaswegians and those newer residents from further afield, who are now calling Glasgow home, whose impact on drinking behaviours has not yet been assessed.

\section{Section 5.5 Conclusion}

To conclude this thesis, I would like to begin with a quote from Mandelbaum's article entitled "Alcohol and Culture":

Drinking patterns give one set of answers to fundamentally mental questions that must be answered in every culture. Drinking is inescapably relevant to attitudes toward bodily sensations. It is made relevant by most peoples to relations between man and woman, to the proper interchange between man and man, and to the nexus between man and god. (1965: 283)

This thesis explored and related the findings of interviews held with mature women in Glasgow regarding mature women's alcohol use. As previously stated, studies of women and alcohol use have largely concentrated on younger women, and this thesis has been my attempt to contribute, in some small way, to the current literature and hopefully draw attention to the lived realities of women in Glasgow who have not yet had an opportunity to share their experiences and perceptions of their changing relationship with alcohol. I would add that neglect of older women in this context may, in fact, reflect the already mentioned tendency of Western society to frame women only as child bearers or mothers. When these roles are no longer anticipated or, are as children become more independent and mothers spend less time with them, we are left with little context with which to value and learn from women. 
In the process of researching and preparing this thesis, I am struck by how alcohol consumption emerges as such an active agent in the lives of these women. From the sensations experienced by an individual body with the first sip of alcohol and its pleasing (or negative) effects, to its manifestation as a representation of gender, class and identity, to the health and social relation on which it hinges, alcohol usage is fraught with challenges and meaning. It is an active agent in people's lives; it is not only acted upon, but also returns that action through the meaning making that occurs when it is incorporated into everyday lives. Alcohol consumption carries the weight of hegemonic discourses on masculinity and femininity and, as has been discussed within these pages, can be used to support or resist these discourses. While research of women's alcohol consumption in Glasgow is still in its early stages, I believe that it has the potential to illuminate a variety of sociocultural issues that have a deep impact on women and how they live their daily lives. This requires a more in-depth look at mature women and their journey along their life course, and more research into their alcohol use and what it can tell us about how cultural ideals and the lived realities of women are changing within this context and just how far from the nexus the affects of such changes may be felt. Their knowledge and experiences could provide us with newly emerging perspectives from which to view health, wellbeing and related factors that impact on their lives, and on the society and culture in which they live.

In closing, I would like to leave the reader with my final thoughts on 'doing' anthropology'. As noted earlier in this thesis, I am an 'anthropologist-in-training', and as such, I would like to offer the following observations. Engaging in fieldwork is uncomfortable. It makes one step outside one's comfort zone like no other experience I have ever encountered. What is enormously helpful is when one engages in self-reflection during the process itself, as it slowly dawns that fieldwork is a skill that is acquired through the 'doing'. In other words, it is not a skill 
that can be learned through books or lectures alone, as it is the experience of the 'process' one undergoes when doing fieldwork that provides the multitude of skills (i.e., interviewing, listening, recruiting etc.) that are required to complete anthropological research effectively. It is this 'process' that is harnessed in fieldwork, and the exploration of a variety of subjects such as history, sociology, economics, politics and public health, etc., that combine to provide the raw data needed so that the anthropology can uncover the construction and roots of meaning embodied within the thoughts and actions of a group of people in a particular place and time. It is this unique intersection of aspects that make the anthropological perspective both informative and important in the study of humanity. 


\section{References}

Alcohol Focus. (2013). Minimum Pricing. http://www.alcohol-focus-scotland.org.uk/minimumpricing Accessed on July 18, 2013.

BBC News. (2013). "Minimum pricing: Challenge to Scottish alcohol legislation fails" (http://www.bbc.co.uk/news/uk-scotland-scotland-politics-22394438) Accessed on May $5,2013$.

Baer, H. A., Singer, M., \& and Johnsen, J.H. (1986). Toward a Critical Medical Anthropology, Social Science \& Medicine, 23(2), 95-98.

Bell, I. (2012, September 12). Cost of alcohol problems revealed. The Herald (Scotland). Retrieved September 12, 2012, from http://www.heraldscotland.com/news/homenews/cost-of-alcohol-problems-revealed.18856489

Bennett, J. (2013). Chav-spotting in Britain: the representation of social class as private choice. Social Semiotics, 23(1), 146-162.

Bennett, L. A. \& Cook Jr., Paul, W. (1996). Alcohol and Drug Studies. In Carolyn F. Sargent and Thomas M. Johnson (Eds.), Medical Anthropology: Contemporary Theory and Method, Revised Edition (pp. 235-251). London: Praeger.

Bernard, H. Russell. (2011). Chapter 8: Interviewing I: Unstructured and Semistructured. In Research Methods in Anthropology: Qualitative and Quantitative Approaches $\left(5^{\text {th }}\right.$ ed.) (pp. 156-186). Lanham: Altamira Press.

Bloor, M., Gannon, M., Hay, G., Jackson, G., Leyland, A.H. \& McKeganey, N. (2008). Contribution of problem drug users' deaths to excess mortality in Scotland: Secondary Analysis of Cohort Study. BMJ, 337, a478. doi: 10.1136/bmj.a478 , n.p. 
Bourdieu, P. (1990). The Logic of Practice. Trans. by Richard Nice. Stanford: Stanford University Press.

Bradley, J. (2006). Sport and the Contestation of Ethnic Identity: Football and Irishness in Scotland. Journal of Ethnic and Migration Studies, 32(7), 1189-1208.

Bradley, J. (1996). Symbol of Prejudice: Football and the Catholic Community in Scotland. Patterns of Prejudice, 30(3), 35-48.

Bruce, S. (2011, April 24). Scottish sectarianism? Let's lay this myth to rest. The Guardian. Retrieved August 28, 2013, from http://www.guardian.co.uk/commentisfree/belief/2011/apr/24/scotland-sectarianismresearch-data

Butler, J. (1988). Performative Acts and Gender Constitution: An Essay in Phenomenology and Feminist Theory, Theatre Journal, 40(4), 519-531.

Cambridge Advanced Learner's Dictionary \& Thesaurus. (2013). Cambridge University Press, http://dictionary.cambridge.org/dictionary/british/ladette Accessed on June 16, 2013.

Damer, S. (1990). Chapter Two: People and Work. In Sean Damer (Au.), Glasgow: going for a song (pp. 21-70). London: Lawrence \& Wishart.

Day, K., Gough, B. \& McFadden, M. (2004). "WARNING! ALCOHOL CAN SERIOUSLY DAMAGE YOUR FEMININE HEALTH" A discourse analysis of recent British newspaper coverage of women and drinking. Feminist Media Studies, 4(1), 165-183.

Dietler, M. (2006). Alcohol: Anthropological/Archaeological Perspectives. Annual Review of Anthropology, 35, 239-249. 
Elvins, Martin. (2009). Chapter Three: Scotland. In Philip Hadfield (Ed.), Nightlife and Crime: Social Order and Governance in International Perspective (pp. 50-63). Oxford: Oxford University Press.

Emslie, C., Hunt, K., \& Lyons, A. (2012). Older and wiser? Men's and women's accounts of drinking in early mid-life. Sociology of Health \& Illness, 34(4), 481-496.

Emslie, C., Lewars, H., Batty, G.D. \& Hunt, K. (2009a). Are there gender differences in levels of heavy, binge and problem drinking? Evidence from three generations in the west of Scotland. Public Health, 123(1), 12-14.

Emslie, C. \& Mitchell, R. (2009b). Are there gender differences in the geography of alcoholrelated mortality in Scotland? An ecological study. BMC Public Health, 9(58), n.p.

Evening Times, The. (2012a). "EU status of Indie Scots 'uncertain"” Monday, Sept. 10, 2012, http://www.eveningtimes.co.uk/news/eu-status-of-indie-scots-uncertain.18831809 Last accessed on May 7, 2013.

Evening Times, The. (2012b). "EU membership claim 'without foundation"' Wednesday, Dec. 12, 2012, http://www.eveningtimes.co.uk/news/eu-membership-claim-withoutfoundation-109750n.19655984 Last accessed on May 7, 2013.

Farmer, P. (2004). Sidney W. Mintz Lecture for 2001, An Anthropology of Structural Violence. Current Anthropology, 45(3), 305-325.

Fetterman, D.M. (2010). Ethnography: Step-by-step ( $3^{\text {rd }}$ ed.), Applied Social Research Methods Series, Volume 17. London: Sage Publications.

Forsey, M.G. (2010). Ethnography as participant listening. Ethnography, 11(4), 558-572. 
Foucault, M. (1978). Part Five: Right of Death and Power over Life. In The History of Sexuality, Volume 1: An Introduction (trans. by Robert Hurley), New York, Pantheon Books (pp. 135-159). http://www.google.ca/search?sourceid=ie7\&q=foucault-the-history-ofsexuality-volume-1.pdf Accessed on June 16, 2013.

Gairns, D. (2012, September 7). Drinks on us? Metro, n.p.

Gardham, M. (2012, September 29). Europe threat to Holyrood's alcohol policy. The Herald (Scotland), p. 2. Retrieved July 24, 2013, from http://www.heraldscotland.com/politics/political-news/europe-threat-to-holyroodsalcohol-policy.19009093

Glasgow City Council. (2013). History of Glasgow: An overview of Glasgow's history from the Stone Age to the present day. http://www.glasgow.gov.uk/index.aspx?articleid=2943

Gordon, R., Heim, D. \& MacAskill, S. (2012). Rethinking drinking cultures: A review of drinking cultures and a reconstructed dimensional approach. Public Health, 126(1), 3-11.

Gose, P. (1994). Deathly Waters and Hungry Mountains. Toronto: University of Toronto Press.

Hanlon, P., Lawder, R.S., Buchanan, A., Redpath, D., Walsh, D., Wood, R., Bain, M., Brewster, D.H., and Chalmers, J. (2005). Why is mortality higher in Scotland than in England and Wales? Decreasing influence of socioeconomic deprivation between 1981 and 2001 supports the existence of a 'Scottish Effect. Journal of Public Health, 27(2), 199-204.

Heath, D.B. (1987). Anthropology and Alcohol Studies: Current Issues. Annual Review of Anthropology, 16, 99-120.

Hendry, J. (1997). Chapter 8: Drinking and Gender in Japan. In Maryon McDonald (Ed.), Gender, Drink and Drugs: Cross Cultural Perspectives on Women (pp. 175-190). New York: Berg. 
Holloway, S.L., Valentine, G. \& Jayne, M. (2009). Masculinities, femininities and the geographies of public and private drinking landscapes. Geoforum, 40, 821-831.

Holtzman, J. (2009). Uncertain Tastes: Memory, Ambivalence and the Politics of Eating in Samburu, Northern Kenya. Berkeley: University of California Press.

Hunt, G. \& Barker, J.C. (2001). Socio-cultural anthropology and alcohol and drug research: towards a unified theory. Social Science \& Medicine, 53(2), 165-188.

Jackson, C. and Tinkler, P. (2007). 'Ladettes' and 'Modern Girls': 'troublesome' young femininities. The Sociological Review, 55(2), 251-271.

Jarvinen, M. (2012). A will to health? Drinking, risk and social class. Health, Risk and Safety, $14(3), 241-256$.

Keep Well Scotland. (2013). Keep Well: Alcohol. http://www.keepwellscotland.org.uk/guide/alcohol.aspx Accessed on May 5, 2013.

Krumeich, A., Weijts, W., Reddy, P. \& Meijer-Weitz, A. (2001). The benefits of anthropological approaches to health and promotion research and practice. Health Education Research, 16(2), 121-130.

Lahelma, E., Lallukka, T., Lassksonen, M., Martikainen, P., Rahlonen, O., Chandola, T., Head, J., Marmot, M., Kagamimori, S., Tatsuse, T. \& Sekine, M. (2010). Social class differences in health behaviours among employees in Britain, Finland and Japan: The influence of psychosocial factors, Health \& Place, 16, 61-70.

Lau-Barraco, C. \& Collins, R.L. (2011). Social networks and alcohol use among nonstudent emerging adults: A preliminary study. Addictive Behaviours, 36(1/2), 47-54. 
Licensing (Scotland) Act 2005. Section, Part 1: Core Provisions, Section 4: The licensing objectives. http://www.legislation.gov.uk/asp/2005/16/section/4 Accessed on July 18, 2013.

Lyons, A.C., Dalton, S.I., \& Hoy, A. (2006). 'Hardcore Drinking': Portrayals of Alcohol Consumption in Young Women's and Men's Magazines. Journal of Health Psychology, 11(2) 223-232.

Lyons, A. C. \& Willott, S. A. (2008). Alcohol Consumption, Gender Identities and Women's Changing Social Positions. Sex Roles, 59 (9/10), 697-712.

Macdonald, S. (1997). Whisky, Women and the Scottish Drink Problem. In Maryon McDonald (Ed.), Gender, Drink and Drugs: Cross Cultural Perspectives on Women (pp. 125-143). New York: Berg.

Madeline McCann. (2013, April 20). The Guardian. Retrieved April 20, 2013, from www.theguardian.com/uk/madeleinemccann

Mandelbaum, D.G. (1965). Alcohol and Culture. Current Anthropology, 6(3), 281-288.

Matheson, Jennifer L. (2007). The Voice Transcription Technique: Use of Voice Recognition Software to Transcribe Digital Interview Data in Qualitative Research. The Qualitative Report, 12(4), 547-560.

Maver, I. (2000). Glasgow. Edinburgh: Edinburgh University Press.

McCartney,G. , Collins,C., Walsh, D. \& Batty, D (2011). Accounting for Scotland's Excess Mortality: Towards a Synthesis. Glasgow: Glasgow Centre for Population Health.

McDonald, M. (1997). Introduction: A Social-Anthropological View of Gender, Drink and Drugs. In Maryon McDonald (Ed.), Gender, Drink and Drugs: Cross Cultural Perspectives on Women (pp. 1-32). New York: Berg. 
Midanik, L. \& Room, R. (2005). Contributions of social science to the alcohol field in an area of biomedicalization. Social Science \& Medicine, 60(5), 1107-1116.

Mozley, A. V. (1977). Introduction to the Dover Edition. In Thomas Annan: Photographs of the Old Closes and Streets of Glasgow, 1868/1877, (pp. v-xii). New York: Dover Publications Inc.

NFO Social Research. (2003). Sectarianism in Glasgow -final report. Prepared for: Glasgow City Council, 825A, January. http://www.glasgow.gov.uk/CHttpHandler.ashx?id=9735\&p=0 Accessed on May 9, 2013.

NHS Health Scotland. (2013). Alcohol Minimum Pricing. http://www.healthscotland.com/topics/health/alcohol/MinimumPricing.aspx Accessed on May 5, 2013.

O’Neill, M. (2006). Museums and Identity in Glasgow. International Journal of Heritage Studies, 12(1), 29-48.

Pacione, M. (1995). Chapter Ten: Popular Images of the City and its People. In Glasgow: The Socio-spatial Development of the City (pp. 236-254). Chichester: John Wiley \& Sons.

Plant, M. (1997). Women and Alcohol: Contemporary and Historical Perspectives. London: Free Association Books.

Plant, M. (2008). The role of alcohol in women's lives: a review of issues and responses. Journal of Substance Use, 13(3), 155-191.

Popham, F., Boyle, P.J., O’Reilly, D. \& Leyland, A.H. (2011). Selective internal migration. Does it explain Glasgow's worsening mortality record? Health \& Place, 17(6), 1212-1217. 
Rolfe, A., Orford, J. \& Dalton, S. (2009). Women, Alcohol and Femininity: A Discourse Analysis of Women Heavy Drinker's Accounts. Journal of Health Psychology, 14(2), 326-335.

Room, R. (2001). Intoxication and bad behaviour: understanding cultural differences in the link. Social Science \& Medicine, 53(2), 189-198.

Room, R., Agar, M., Beckett, J., Bennet, L.A., Casswell, S., Heath, D.B., Leland, J., Levy, J.E., Madsen, W., Marshall, M., Moskalewicz, J.C.N., Rodin, M.B., Sackett, L., Sargent, M., Strug, D., Waddell, J.O. (1984). Alcohol and Ethnography: A Case of Problem Deflation? [and Comments and Reply]. Current Anthropology, 25(2), 169-191.

Rudolfsdottir, A. G. \& Morgan, P. (2009). 'Alcohol is my Friend': Young Middle Class Women Discuss their Relationship with Alcohol. Journal of Community \& Applied Social Psychology, 19(6), 492-505.

Ryan, G.W. \& Bernard, H.R. (2003). Techniques to Identify Themes. Field Methods, 15(1), 85109.

Scholte, B. (1969). Towards a Reflexive and Critical Anthropology. In Dell Hymes (Ed.), Reinventing Anthropology (pp. 430-457). New York: Pantheon.

Scheper-Hughes, N. \& Lock, M.M. (1987). The Mindful Body: A Prolegomenon to Future Work in Medical Anthropology. Medical Anthropology Quarterly, New Series, 1(1), 641.

Scheper-Hughes, N. \& Lock, M.M. (1996). A Critical-Interpretive Approach in Medical Anthropology: Rituals and Routines of Discipline and Dissent. In Carolyn F. Sargent and Thomas M. Johnson (Eds.) Medical Anthropology: Contemporary Theory and Method, Revised Edition (pp. 41-70). London: Praeger. 
Scottish Government, The. (2009). Changing Scotland's Relationship with Alcohol: A Framework for Action. Produced for the Scottish Government by RR Donnelley. Published by the Scottish Government, February 2009. http://www.scotland.gov.uk/Resource/Doc/262905/0078610.pdf Accessed on July 18, 2013.

Seaman, P. and Edgar, F. (2012). Creating Better Stories: Alcohol and gender in transitions to adulthood. Glasgow: Glasgow Centre for Population Health. http://www.academia.edu/1472863/Creating_better_stories_Alcohol_and_gender_in_tran sitions_to_adulthood Accessed on June 16, 2013.

Singer, M. (1986). Developing a Critical Perspective in Medical Anthropology. Medical Anthropology Quarterly, 17(5), 128-129.

Smith, E. (2012, September 12). The rise of the middle class NEDS: City's teens look up to rebels. The Glaswegian, n.p.

Singer, M. (2004). The social origins and expressions of illness. British Medical Bulletin, 69, 919.

Thom, B. (1997). Women and Alcohol: The Emergence of a Risk Group. In Maryon McDonald (Ed.), Gender, Drink and Drugs: Cross Cultural Perspectives on Women (pp. 33-54). New York: Berg.

Walsh, D., Bendel, N., Jones, R. \& Hanlon, P. (2010a). Investigating a 'Glasgow Effect': Why do equally deprived UK cities experience different health outcomes? Glasgow: Glasgow Centre of Population Health.

Walsh, D., Bendel, N., Jones, R. \& Hanlon, P. (2010b). It's not 'just deprivation': Why do equally deprived UK cities experience different health outcomes? Public Health, 124(9), 487-495.

World Health Organization. (2012). Social Determinants of Health, http://www.who.int/social_determinants/en/ Accessed on June 29, 2013. 
Zilberman, M., Tavares, H. \& El-Guebaly, N. (2008) Gender Similarities and Differences. Journal of Addictive Diseases, 22(4), 61-74. 


\section{Appendix A: Interview Questions}

\section{Demographic Questions}

1. Why did you volunteer to participate in this study?

2. For how many years have you lived in Glasgow/Greater Glasgow area?

3. Which age category applies to you? (Please circle one)

- 25-34

- $35-44$

- $45-54$

- $55-65$

4. What word best describes your current marital status? (Please circle one)

- Single

- Married

- Living with someone

- Divorced/Separated

- Widowed

5. Do you have children? (Please circle one)

- Yes (please specify number of children and their ages)

- No

6. What is your current occupation? (Please note: If retired please put write in Retired-and the last job you had)

7. What is the highest level of education you have received?

\section{Introductory Questions}

- In general, what kind of relationship do people in Glasgow have with alcohol? [Probe: What makes you think or believe this? Is there any particular group of people you are thinking of here? Who? Why do you single out this group?]

- What would you say people in your community think about alcohol use in Glasgow?

- Where do you see or hear stories about alcohol use in your community? [Probe: Can you give me an example that describes what you have witnessed or read about, or heard through others. How did you react to this?]

- What is the usual way that Glaswegians get news and/or information about alcohol use? 


\section{Transition Questions}

- For your generation, could you explain to me the way women used alcohol when you were growing up?

- Where did women drink back then? Were their places women couldn't go to drink back then?

- What was considered an 'average' level of drinking for a woman?

\section{$\underline{\text { Key Questions }}$}

- Under what circumstances do women of your age group drink today?

- Do you think that women's drinking practices have changed over the past 10 to 20 years? (If yes, how have they changed?)

- What do you know about alcohol's impact on women?

- What factors influence women's use of alcohol in Glasgow?

- If you wanted to find out more information on alcohol use and its benefits or risks, where would you look?

\section{Summary Questions}

- Overall, how do you view women's drinking habits in Glasgow? Does this view include women in all age groups? [Could you tell me a bit more about that?]

- On what do you base your views of women's alcohol consumption?

- Do the views you have mentioned seem to be shared among your friends?

- How do such beliefs or common knowledge about women's use of alcohol come about? [Probe: Where do they come from? Where do you see such images? Stereotyping or assumptions embedded in such perceptions?]

\section{Closing Question}

- Is there anything else you would like to share with me about women and alcohol?

- Do you have any questions for me?

- Finally, what information would you like to receive from me when the project has been completed? 